\title{
Enantioselective Total Synthesis of (-)-Heptemerone B and (-)-Guanacastepene E
}

\author{
Aubry K. Miller, Chambers C. Hughes, Joshua J. Kennedy-Smith, Stefan N. Gradl and Dirk Trauner* \\ Department of Chemistry, University of California—Berkeley, Berkeley, California 94720
}

\section{Table of Contents}

1) General information $\quad$ S1

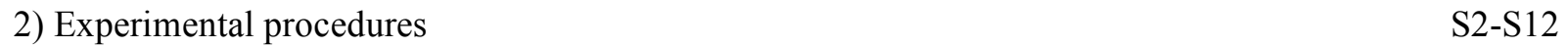

3) NMR spectra of selected compounds $\quad$ S13-S50

$\begin{array}{ll}\text { 4) X-ray tables } & \text { S51-S80 }\end{array}$

\section{General information:}

Unless otherwise noted, all reagents were purchased from commercial suppliers and used without further purification. Specifically, lithium 2-thienylcyanocuprate [2-(thienyl) $\mathrm{Cu}(\mathrm{CN}) \mathrm{Li}$ ] was purchased as a 0.5 M solution in THF from Aldrich, and reticulated vitreous carbon (RVC) was purchased from Electrolytica, Inc. Toluene $(\mathrm{PhMe})$, Diethyl ether $\left(\mathrm{Et}_{2} \mathrm{O}\right)$ and dichloromethane $\left(\mathrm{CH}_{2} \mathrm{Cl}_{2}\right)$ were dried according to the procedure described by Bergman. ${ }^{1}$ Tetrahydrofuan (THF) was distilled from Na/benzophenone. HMPA and $\mathrm{BF}_{3} \cdot \mathrm{OEt}_{2}$ were distilled from $\mathrm{CaH}_{2}$ and stored in Schlenk flasks over $4 \AA$ molecular sieves. 2,6-Lutidine, methanol $(\mathrm{MeOH})$, triethylamine $\left(\mathrm{Et}_{3} \mathrm{~N}\right)$ and diisopropylamine $\left(i-\mathrm{Pr}_{2} \mathrm{NH}\right)$ were distilled from calcium hydride $\left(\mathrm{CaH}_{2}\right)$. Tetra- $n$ butylammonium fluoride (TBAF) was purchased as a 1.0 M solution in THF and stored in a Schlenk flask over $4 \AA$ molecular sieves. $n$-Butyl lithium was titrated using diphenylacetic acid in THF. $t$-Butyl lithium was titrated

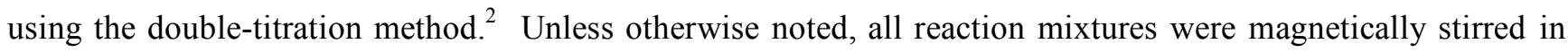
oven-dried glassware under a blanket of argon. External bath temperatures were used to record all reaction mixture temperatures. Extracts were dried over anhydrous $\mathrm{MgSO}_{4}$ and solvents were removed with a rotary evaporator at aspirator pressure. Analytical thin layer chromatography (TLC) was carried out on Merck silica gel $60 \mathrm{~F}_{254}$ TLC plates. TLC visualization was accomplished using $254 \mathrm{~nm}$ UV light or charring solutions of $p$ anisaldehyde (PAA). Flash chromatography was performed on ICN siliTech 32-63 D $60 \AA$ silica gel according to the procedure of Still. ${ }^{3}$ Melting points were measured on a Büchi melting point apparatus and are uncorrected. ${ }^{1} \mathrm{H}$ - and ${ }^{13} \mathrm{C}$ NMR spectra were recorded on a Bruker DRX-500, AV-500 or AVB-400 spectrometer in $\mathrm{CDCl}_{3}$ with spectra calibrated to $\mathrm{CHCl}_{3}(\delta 7.26)$ and $\mathrm{CDCl}_{3}(\delta$ 77.23). Infrared spectra (IR) were obtained on $\mathrm{NaCl}$ plates with an ATI Mattson Gemini FTIR or a Nicolet MAGNA-IR 850 spectrometer. High-resolution mass spectra (HRMS) were obtained on a VG ProSpec ZAB2-EQ Mass Spectrometer using fast atom bombardment (FAB) or electron impact (EI) at $70 \mathrm{eV}$. Elemental analysis was performed by the Microanalytical Laboratory 
operated by the UCB College of Chemistry. X-ray analysis was performed on a Bruker SMART CCD areadetector diffractometer.

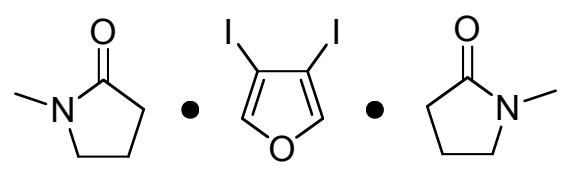

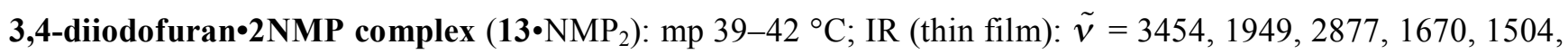
1473, 1459, 1440, 1403, 1301, 1264, 1125, 1114, 1040, 940, $865 \mathrm{~cm}^{-1} ;{ }^{1} \mathrm{H}$ NMR (500 MHz): $\delta=7.43$ (s, $\left.2 \mathrm{H}\right)$, $3.37(\mathrm{t}, J=7.25 \mathrm{~Hz}, 4 \mathrm{H}), 2.83(\mathrm{~s}, 6 \mathrm{H}), 2.36(\mathrm{t}, J=8.00 \mathrm{~Hz}, 4 \mathrm{H}), 2.02$ (app quint, $J=7.5 \mathrm{~Hz}, 4 \mathrm{H}) ;{ }^{13} \mathrm{C} \mathrm{NMR}$ (125 MHz): $\delta=174.7,146.1,77.4,49.2,30.5,29.4,17.5$.

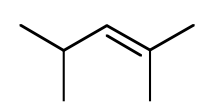

2,4-dimethyl-2-pentene (15): To a suspension of isopropyltriphenylphosphonium bromide (97.20 g, 252.3 mmol) in $\mathrm{Et}_{2} \mathrm{O}(400 \mathrm{~mL})$ was added a solution of $n$-BuLi $(120.0 \mathrm{~mL}, 240.0 \mathrm{mmol}, 2.0 \mathrm{M}$ in pentane $)$ at $0{ }^{\circ} \mathrm{C}$ over $10 \mathrm{~min}$. After the addition was complete, the resulting red solution was allowed to warm to rt. After $10 \mathrm{~h}$, isobutyraldehyde $(20.0 \mathrm{~mL}, 218.8 \mathrm{mmol})$ was added dropwise. After the addition was complete, the mixture was heated at reflux. After $24 \mathrm{~h}$, the volatile components of the reaction mixture were fractionally distilled at reduced pressure to give a crude mixture of the desired product mixed with $\mathrm{Et}_{2} \mathrm{O}$ and pentane. This mixture was fractionally distilled by vacuum transfer to give $4.42 \mathrm{~g}$ of a $75 \% \mathrm{w} / \mathrm{w}$ solution (15\% yield) of 15 in $\mathrm{Et}_{2} \mathrm{O}:{ }^{1} \mathrm{H}$ NMR (400 MHz): $\delta=4.94(\mathrm{~d}, J=8.8 \mathrm{~Hz}, 1 \mathrm{H}), 2.48(\mathrm{~m}, 1 \mathrm{H}), 1.67$ (s, $3 \mathrm{H}), 1.61$ (s, $3 \mathrm{H}), 0.94(\mathrm{~d}, J=6.0 \mathrm{~Hz}, 6$ $\mathrm{H})$.

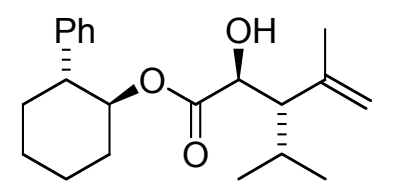

(2S,3S)-(1S,2R)-2-phenylcyclohexyl 2-hydroxy-3-isopropyl-4-methylpent-4-enoate (26): To a solution of gloxylate $25(1.478 \mathrm{~g}, 6.36 \mathrm{mmol})$ in $\mathrm{CH}_{2} \mathrm{Cl}_{2}(25.0 \mathrm{~mL})$ was added a solution of 15 (1.00 g, $7.64 \mathrm{mmol}, 75 \%$ w/w in $\mathrm{Et}_{2} \mathrm{O}$ ) at $-78{ }^{\circ} \mathrm{C}$ under $\mathrm{N}_{2}$. After $10 \mathrm{~min}$, a solution of $\mathrm{SnCl}_{4}\left(7.0 \mathrm{~mL}, 7.0 \mathrm{mmol}, 1.0 \mathrm{M}\right.$ in $\left.\mathrm{CH}_{2} \mathrm{Cl}_{2}\right)$ was added dropwise. After $4 \mathrm{~h}$, the reaction mixture was quenched with 1:1:1 saturated aqueous $\mathrm{NaHCO}_{3}-$ saturated aqueous Rochelle's salt $-\mathrm{H}_{2} \mathrm{O}(150 \mathrm{~mL})$ and diluted with $\mathrm{CH}_{2} \mathrm{Cl}_{2}(100 \mathrm{~mL})$. The two layers were separated and the aqueous layer was extracted with $\mathrm{CH}_{2} \mathrm{Cl}_{2}(2 \times 100 \mathrm{~mL})$. The combined organic extracts were dried, filtered and concentrated. The products were purified by column chromatography (10\% EtOAc in hexanes) to yield 1.35 
g (64\% yield) of 26 as a 10:1 mixture with a diastereomer. Racemic 26 could be purified by crystallization from hexanes at $-20{ }^{\circ} \mathrm{C}$ and was found to be amenable to single crystal X-ray analysis. The enantiopure material, however, was not crystalline and had to be carried on as a mixture of diastereomers until it was converted into cyclopentenone $\mathbf{2 9}$ at which point it could be quantitatively separated from the undesired diasteromer. Therefore, the characterization data for compounds $\mathbf{2 6}, \mathbf{S 1}, \mathbf{2 7}$, and $\mathbf{2 8}$ are of racemic material: $\mathrm{R}_{f} 0.30(10 \%$ EtOAc in hexanes); mp 76-77 ${ }^{\circ} \mathrm{C}$; IR (thin film): $\tilde{v}=3500,2935,2861,1729 \mathrm{~cm}^{-1} ;{ }^{1} \mathrm{H}$ NMR $(500 \mathrm{MHz}): \delta=7.29-7.26$ (m, 2 H), 7.17-7.21 (m, 3 H), $4.81(\mathrm{dt}, J=4.3 \mathrm{~Hz}, 10.7 \mathrm{~Hz}, 1 \mathrm{H}), 4.48(\mathrm{~s}, 1 \mathrm{H}), 4.23$ (dd, J = 2.9 Hz, 7.0 Hz, 1 H), $3.83(\mathrm{~s}, 1 \mathrm{H}), 2.72(\mathrm{~m}, 1 \mathrm{H}), 2.44(\mathrm{~d}, J=6.8 \mathrm{~Hz}, 1 \mathrm{H}), 2.30(\mathrm{~m}, 1 \mathrm{H}), 1.96-1.91(\mathrm{~m}, 1 \mathrm{H}), 1.88-1.76(\mathrm{~m}, 3 \mathrm{H})$, $1.65-1.56(\mathrm{~m}, 1 \mathrm{H}), 1.52-1.43(\mathrm{~m}, 2 \mathrm{H}), 1.40-1.33(\mathrm{~m}, 2 \mathrm{H}), 1.31(\mathrm{~s}, 3 \mathrm{H}), 0.94(\mathrm{~d}, J=6.5 \mathrm{~Hz}, 3 \mathrm{H}), 0.71(\mathrm{~d}, J=$ $6.6 \mathrm{~Hz}, 3 \mathrm{H}) ;{ }^{13} \mathrm{C}$ NMR $(100 \mathrm{MHz}): \delta=174.4$, 143.2, 143.2, 128.7, 127.9, 126.9, 114.4, 78.8, 71.5, 56.6, 49.5, 33.8, 32.2, 27.0, 25.9, 24.7, 22.4, 21.4, 20.7; HRMS (EI): calcd for $\mathrm{C}_{21} \mathrm{H}_{30} \mathrm{O}_{3}(\mathrm{M})^{+}:$330.2195; found: 330.2188; Anal. calcd for $\mathrm{C}_{21} \mathrm{H}_{30} \mathrm{O}_{3}$ : C 76.33, H 9.15; found: C 76.32, $\mathrm{H} 9.33$.

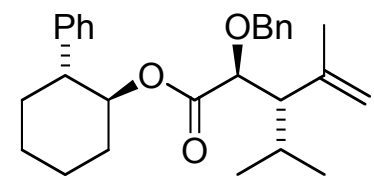

(2S,3S)-(1S,2R)-2-phenylcyclohexyl 2-(benzyloxy)-3-isopropyl-4-methylpent-4-enoate (S1): To a suspension of $\mathrm{NaH}(275 \mathrm{mg}, 6.88 \mathrm{mmol}, 60 \%$ in oil) in THF (30 mL) was added a solution of $\mathbf{2 6}(1.515 \mathrm{~g}$, $4.584 \mathrm{mmol})$ in THF ( $5 \mathrm{~mL}+$ two $5 \mathrm{~mL}$ rinses) at $0{ }^{\circ} \mathrm{C}$. After the addition was complete, benzyl bromide $(0.58 \mathrm{~mL}, 4.85 \mathrm{mmol})$ and tetra- $n$-butylammonium iodide $(170 \mathrm{mg}, 0.46 \mathrm{mmol})$ were added. After $10 \mathrm{~min}$, the mixture was allowed to warm to rt. After $16 \mathrm{~h}$, the reaction mixture was quenched with half saturated brine $(300 \mathrm{~mL})$ and diluted with EtOAc $(100 \mathrm{~mL})$. The two layers were separated and the aqueous layer was extracted with EtOAc $(2 \times 50 \mathrm{~mL})$. The combined organic extracts were dried, concentrated and purified by column chromatography (7\% EtOAc in hexanes) to give $1.93 \mathrm{~g}$ (100\% yield) of $\mathbf{S} \mathbf{1}$ as a thick colorless oil: $\mathrm{R}_{f} 0.54$ (10\% EtOAc in hexanes); IR (thin film): $\tilde{v}=2934,2860,1745,1723 \mathrm{~cm}^{-1} ;{ }^{1} \mathrm{H}$ NMR $(500 \mathrm{MHz}): \delta=7.36-7.17(\mathrm{~m}, 10 \mathrm{H}), 5.17(\mathrm{dt}, J=4.2 \mathrm{~Hz}$, $10.6 \mathrm{~Hz}, 1 \mathrm{H}), 4.71(\mathrm{~s}, 1 \mathrm{H}), 4.38(\mathrm{~s}, 1 \mathrm{H}), 4.09$ (d, $J=11.8,1 \mathrm{H}), 3.99$ (d, $J=2.9 \mathrm{~Hz}, 1 \mathrm{H}), 3.77(\mathrm{~d}, J=11.8 \mathrm{~Hz}$, $1 \mathrm{H}), 2.79$ (m, $1 \mathrm{H}), 2.28(\mathrm{~m}, 1 \mathrm{H}), 2.20-1.88(\mathrm{~m}, 4 \mathrm{H}), 1.85-1.79(\mathrm{~m}, 1 \mathrm{H}), 1.78(\mathrm{~s}, 3 \mathrm{H}), 1.62-1.38(\mathrm{~m}, 4 \mathrm{H})$, 0.75 (m, $6 \mathrm{H}) ;{ }^{13} \mathrm{C}$ NMR (125 MHz): $\delta=172.3,144.2,143.5,138.3,128.5,128.1,127.7,127.5,127.4,126.6$, 115.2, 79.7, 76.6, 72.0, 57.6, 49.8, 34.8, 32.2, 25.9, 25.9, 24.8, 21.2, 20.7, 20.4; HRMS (FAB $\left.{ }^{+}\right)$: calcd for $\mathrm{C}_{28} \mathrm{H}_{37} \mathrm{O}_{3}(\mathrm{M}+\mathrm{H})^{+}:$421.2743; found: 421.2743 .

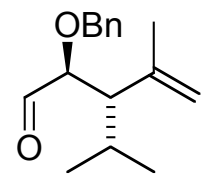


(2S,3S)-2-(benzyloxy)-3-isopropyl-4-methylpent-4-enal (27): To a solution of $\mathbf{S 1}(300 \mathrm{mg}, 0.713 \mathrm{mmol})$ in $\mathrm{CH}_{2} \mathrm{Cl}_{2}(7.0 \mathrm{~mL})$ was added a solution of DIBAL $(0.90 \mathrm{~mL}, 1.35 \mathrm{mmol}, 1.5 \mathrm{M}$ in $\mathrm{PhMe})$ dropwise at $-78{ }^{\circ} \mathrm{C}$. After $2.5 \mathrm{~h}$ the reaction was quenched at $-78{ }^{\circ} \mathrm{C}$ with EtOAc $(2.0 \mathrm{~mL})$ and then diluted with $\mathrm{CH}_{2} \mathrm{Cl}_{2}(50 \mathrm{~mL})$ and quarter saturated Rochelle's salt $(100 \mathrm{~mL})$. The resulting mixture was stirred vigorously for $1 \mathrm{~h}$, the two layers were separated and the aqueous layer was extracted with $\mathrm{CH}_{2} \mathrm{Cl}_{2}(2 \times 50 \mathrm{~mL})$. The combined organic extracts were dried, filtered and concentrated. The product was purified by column chromatography (5\% EtOAc in hexanes) to provide (149 mg, 85\% yield) of 27 as a colorless liquid: $\mathrm{R}_{f} 0.43$ (10\% EtOAc in hexanes); IR (thin film): $\tilde{v}=3069,3032,2960,2870,1732,1642,1498,1455,1377 \mathrm{~cm}^{-1} ;{ }^{1} \mathrm{H}$ NMR $(500 \mathrm{MHz}): \delta=9.60(\mathrm{~d}$, $J=1.8 \mathrm{~Hz}, 1 \mathrm{H}), 7.37-7.29(\mathrm{~m}, 5 \mathrm{H}), 4.84$ (br s, $1 \mathrm{H}), 4.75$ (d, $J=12.6 \mathrm{~Hz}, 1 \mathrm{H}), 4.74$ (br s, $1 \mathrm{H}), 4.43$ (d, $J=$ $12.6 \mathrm{~Hz}, 1 \mathrm{H}), 4.00(\mathrm{dd}, J=3.7,1.8 \mathrm{~Hz}, 1 \mathrm{H}), 2.16-2.04(\mathrm{~m}, 2 \mathrm{H}), 1.78(\mathrm{~s}, 3 \mathrm{H}), 0.90$ (d, J = 6.4 Hz, $3 \mathrm{H}), 0.85$ $(\mathrm{d}, J=6.4 \mathrm{~Hz}, 3 \mathrm{H}) ;{ }^{13} \mathrm{C}$ NMR $(125 \mathrm{MHz}): \delta=205.2,144.0,137.8,128.6,128.0,127.9,115.6,84.3,73.3,56.3$, 26.3, 21.5, 20.9, 20.6; HRMS $\left(\mathrm{FAB}^{+}\right)$: calcd for $\mathrm{C}_{16} \mathrm{H}_{21} \mathrm{O}_{2}(\mathrm{M}-\mathrm{H})^{+}:$: 245.1542; found: 245.1536.

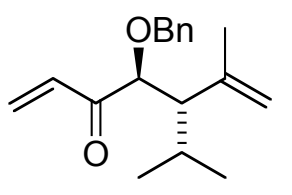

(4S,5S)-4-(benzyloxy)-5-isopropyl-6-methylhepta-1,6-dien-3-one (28): $\mathrm{CeCl}_{3} \cdot 7 \mathrm{H}_{2} \mathrm{O}$ (455 $\mathrm{mg}, 1.22 \mathrm{mmol}$ ) was powdered in a mortar and pestle and heated to $90{ }^{\circ} \mathrm{C}$ at $200 \mathrm{mTorr}$ for $2 \mathrm{~h}$. The temperature was then raised to $140{ }^{\circ} \mathrm{C}$ for $5 \mathrm{~h}$ and then cooled to $0{ }^{\circ} \mathrm{C}$ under argon. THF $(5.0 \mathrm{~mL})$ was added, the mixture was sonicated for $1 \mathrm{~min}$ and then stirred at $\mathrm{rt}$ for $16 \mathrm{~h}$. The suspension was then cooled to $-78{ }^{\circ} \mathrm{C}$ and a solution of vinylmagnesium bromide (1.10 mL, $1.10 \mathrm{mmol}, 1.0 \mathrm{M}$ in THF) was added. After $2 \mathrm{~h}$, a solution of 27 (100 mg, $0.41 \mathrm{mmol})$ in THF $(1.0 \mathrm{~mL}+$ two $0.5 \mathrm{~mL}$ rinses) was added. After $2 \mathrm{~h}$, the reaction was quenched with saturated aqueous $\mathrm{NH}_{4} \mathrm{Cl}(20 \mathrm{~mL})$ and diluted with EtOAc $(10 \mathrm{~mL})$. The mixture was filtered through celite and the two layers were separated. The aqueous layer was extracted with EtOAc $(2 \times 5 \mathrm{~mL})$ and the combined organic extracts were dried, filtered and concentrated. The products were purified by column chromatography $(5 \rightarrow 10 \rightarrow 15 \%$ EtOAc in hexanes) to give $91 \mathrm{mg}$ ( $82 \%$ yield) of the secondary alcohol as a mixture of diastereomers that were taken on without characterization. To a solution of the secondary alcohol $(91 \mathrm{mg}, 0.33$ $\mathrm{mmol}$ ) in $\mathrm{CH}_{2} \mathrm{Cl}_{2}$ (4 mL from a squirt bottle) was added Dess-Martin periodinane (172 $\left.\mathrm{mg}, 0.41 \mathrm{mmol}\right)$ at $\mathrm{rt}$ open to the air. After $2 \mathrm{~h}$, the reaction was quenched with 1:1:1 saturated aqueous $\mathrm{NaHCO}_{3}-$ saturated aqueous $\mathrm{Na}_{2} \mathrm{~S}_{2} \mathrm{O}_{3}-\mathrm{H}_{2} \mathrm{O}(10 \mathrm{~mL})$ and diluted with EtOAc $(10 \mathrm{~mL})$. The two layers were separated and the aqueous layer was extracted with EtOAc $(2 \times 2 \mathrm{~mL})$. The combined organic extracts were dried, filtered and concentrated. The product was purified by column chromatography (5\% EtOAc in hexanes) to give $78 \mathrm{mg}$ ( $86 \%$ yield, $70 \%$ over 2 steps) of 28 as a colorless oil which solidified at $-20{ }^{\circ} \mathrm{C}: \mathrm{R}_{f} 0.35$ (10\% EtOAc in hexanes); IR (thin film): $\tilde{v}=$ 2959, 2927, 2870, 1696, 1612, 1455, $1401 \mathrm{~cm}^{-1} ;{ }^{1} \mathrm{H}$ NMR (500 MHz): $\delta=7.36-7.28$ (m, $\left.5 \mathrm{H}\right), 6.73(\mathrm{dd}, J=17.4$, $10.5 \mathrm{~Hz}, 1 \mathrm{H}), 6.32$ (dd, $J=17.4,1.7 \mathrm{~Hz}, 1 \mathrm{H}), 5.67$ (dd, $J=10.5,1.7 \mathrm{~Hz}, 1 \mathrm{H}), 4.77$ (br s, $1 \mathrm{H}), 4.65-4.62$ (m, 2 
H), $4.29(\mathrm{~d}, J=11.6 \mathrm{~Hz}, 1 \mathrm{H}), 4.23(\mathrm{~d}, J=3.9 \mathrm{~Hz}, 1 \mathrm{H}), 2.11-2.01(\mathrm{~m}, 2 \mathrm{H}), 1.75(\mathrm{~s}, 3 \mathrm{H}), 0.90(\mathrm{~d}, J=6.3 \mathrm{~Hz}, 3$ $\mathrm{H}), 0.83(\mathrm{~d}, J=6.3 \mathrm{~Hz}, 3 \mathrm{H}) ;{ }^{13} \mathrm{C}$ NMR $(125 \mathrm{MHz}): \delta=202.4,144.0,138.1,132.2,128.6,128.5,127.9,127.7$, 115.6, 85.6, 73.1, 57.6, 26.7, 21.5, 21.1, 20.8; HRMS $\left(\mathrm{FAB}^{+}\right)$: calcd for $\mathrm{C}_{18} \mathrm{H}_{24} \mathrm{O}_{2}(\mathrm{M}+\mathrm{Li})^{+}:$279.1936; found: 279.1924 .

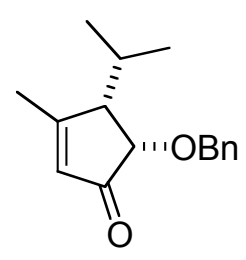

(4S,5S)-5-(benzyloxy)-4-isopropyl-3-methylcyclopent-2-enone (29): To a solution of enantiopure 28 (299 $\mathrm{mg}$, $1.10 \mathrm{mmol}$ of a 10:1 mixture of diastereomers) in PhMe (110 mL, degassed for 30 min with bubbling argon) was added the Grubbs $2^{\text {nd }}$ generation catalyst $(47.8 \mathrm{mg}, 0.056 \mathrm{mmol}$ ). The resulting brown solution was heated to reflux under argon for $2.5 \mathrm{~h}$. The reaction mixture was concentrated and purified by column chromatography (20\% EtOAc in hexanes) to give $206 \mathrm{mg}$ of $\mathbf{2 9}$ and $26 \mathrm{mg}$ of the undesired diastereomer (86\% overall yield), both as colorless oils that solidified at $-20{ }^{\circ} \mathrm{C}$. Data for 29: $\mathrm{R}_{f} 0.34\left(30 \%\right.$ EtOAc in hexanes); $[\alpha]_{\mathrm{D}}-23.7^{\circ}(c=$ 1.19, $\mathrm{CHCl}_{3}$ ); IR (thin film): $\tilde{v}=2962,2934,2874,1713,1612 \mathrm{~cm}^{-1} ;{ }^{1} \mathrm{H}$ NMR $(500 \mathrm{MHz}): \delta=7.41-7.26(\mathrm{~m}, 5$ H), 5.99 (br s, $1 \mathrm{H}), 5.02(\mathrm{~d}, J=12.3 \mathrm{~Hz}, 1 \mathrm{H}), 4.77(\mathrm{~d} J=12.3 \mathrm{~Hz}, 1 \mathrm{H}), 4.03(\mathrm{~d}, J=6.5 \mathrm{~Hz}, 1 \mathrm{H}), 2.87(\mathrm{~m}, 1$ H), 2.25 (dhept, $J=2.7,7.1 \mathrm{~Hz}, 1 \mathrm{H}), 2.07(\mathrm{~s}, 3 \mathrm{H}), 1.03(\mathrm{~d}, J=7.1 \mathrm{~Hz}, 3 \mathrm{H}), 0.85(\mathrm{~d}, J=7.1 \mathrm{~Hz}, 3 \mathrm{H}) ;{ }^{13} \mathrm{C}$ NMR (125 MHz): $\delta=207.6,175.6,138.6,129.8,128.8,128.0,128.0,81.5,73.5,53.9,28.2,21.2,20.6$ 19.4; HRMS $\left(\mathrm{FAB}^{+}\right)$: calcd for $\mathrm{C}_{16} \mathrm{H}_{21} \mathrm{O}_{2}(\mathrm{M}+\mathrm{H})^{+}: 245.1542$; found: 245.1540 .

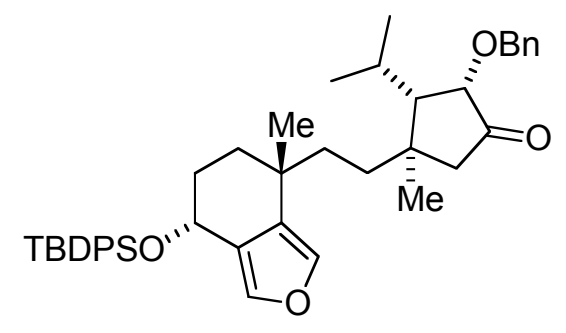

Ketone 31: Iodide 24 (528 mg, $0.97 \mathrm{mmol})$ was dissolved in benzene (15 mL), concentrated and placed under high vacuum (200 mTorr) for $30 \mathrm{~min}$. It was then dissolved in $\mathrm{Et}_{2} \mathrm{O}(10 \mathrm{~mL})$ and cooled to $-78{ }^{\circ} \mathrm{C}$ under argon. To this solution was added a solution of $t$ - BuLi (1.21 mL, $1.94 \mathrm{mmol}, 1.6 \mathrm{M}$ in pentane) dropwise. After $20 \mathrm{~min}$, a solution of 2-(thienyl) $\mathrm{Cu}(\mathrm{CN}) \mathrm{Li}(3.90 \mathrm{~mL}, 0.975 \mathrm{mmol}, 0.25 \mathrm{M}$ in THF) was added dropwise to make a cloudy suspension that eventually produced a sticky yellow precipitate. After $1 \mathrm{~h}$ of vigorous stirring, $\mathrm{BF}_{3} \cdot \mathrm{OEt}_{2}$ $(0.22 \mathrm{~mL}, 1.74 \mathrm{mmol})$ was added dropwise followed by a solution of $29(201 \mathrm{mg}, 0.82 \mathrm{mmol})$ in $\mathrm{Et}_{2} \mathrm{O}(3.0 \mathrm{~mL}+$ two $1.0 \mathrm{~mL}$ rinses). The reaction mixture was warmed to $-40{ }^{\circ} \mathrm{C}$ and, after $1 \mathrm{~h}$, quenched with $9: 1$ saturated aqueous $\mathrm{NH}_{4} \mathrm{Cl}$-concentrated aqueous $\mathrm{NH}_{4} \mathrm{OH}(20 \mathrm{~mL})$ and warmed to rt. The mixture was filtered through 
celite and the two layers were separated. The aqueous layer was extracted with $\mathrm{Et}_{2} \mathrm{O}(2 \times 10 \mathrm{~mL})$. The combined organic extracts were dried, filtered and concentrated. The product was purified by column chromatography $(4 \rightarrow 10 \rightarrow 20 \%$ EtOAc in hexanes) to give $296 \mathrm{mg}$ (54\% yield) of 31 as a sticky glass, $131 \mathrm{mg}$ (24\% yield) of the 1,2 adduct and $20 \mathrm{mg}$ of recovered 29. Data for 31: $\mathrm{R}_{f} 0.34\left(10 \%\right.$ EtOAc in hexanes); $[\alpha]_{\mathrm{D}}-38.8^{\circ}(c=2.50$, $\mathrm{CHCl}_{3}$ ); IR (thin film): $\tilde{v}=2958,2858,1742,1461,1428 \mathrm{~cm}^{-1} ;{ }^{1} \mathrm{H}$ NMR $(500 \mathrm{MHz}): \delta=7.73(\mathrm{~m}, 2 \mathrm{H}), 7.67$ (m, $2 \mathrm{H}), 7.48-7.28(\mathrm{~m}, 11 \mathrm{H}), 7.11(\mathrm{~s}, 1 \mathrm{H}), 6.76(\mathrm{~s}, 1 \mathrm{H}), 4.94(\mathrm{~d}, J=11.7 \mathrm{~Hz}, 1 \mathrm{H}), 4.84(\mathrm{~m}, 1 \mathrm{H}), 4.48(\mathrm{~d}, J=$ $11.7 \mathrm{~Hz}, 1 \mathrm{H}), 3.66$ (d, $J=6.3 \mathrm{~Hz}, 1 \mathrm{H}), 2.34-2.20$ (m, $3 \mathrm{H}), 2.08-2.02(\mathrm{~m}, 1 \mathrm{H}), 1.88-1.81(\mathrm{~m}, 1 \mathrm{H}), 1.74-1.47$ (m $6 \mathrm{H}), 1.30-1.25(\mathrm{~m}, 4 \mathrm{H}), 1.13(\mathrm{~s}, 3 \mathrm{H}), 1.08(\mathrm{~s}, 9 \mathrm{H}), 1.06(\mathrm{~d}, J=6.7 \mathrm{~Hz}, 3 \mathrm{H}), 0.97(\mathrm{~d}, J=6.5 \mathrm{~Hz}, 3 \mathrm{H}) ;{ }^{13} \mathrm{C}$ NMR (125 MHz): $\delta=216.4,139.3,138.3,137.2,136.2,136.1,134.6,134.3,130.3,129.9,129.9,128.4,127.8$, $127.8,127.7,127.7,124.9,81.5,72.4,63.6,56.2,51.7,41.3,38.3,37.8,32.7,31.4,30.1,30.1,27.2,25.2,23.4$, 23.2, 22.7, 19.5; HRMS $\left(\mathrm{FAB}^{+}\right)$: calcd for $\mathrm{C}_{43} \mathrm{H}_{53} \mathrm{O}_{4} \mathrm{Si}(\mathrm{M}-\mathrm{H})^{+}$: 661.3713; found: 661.3714.

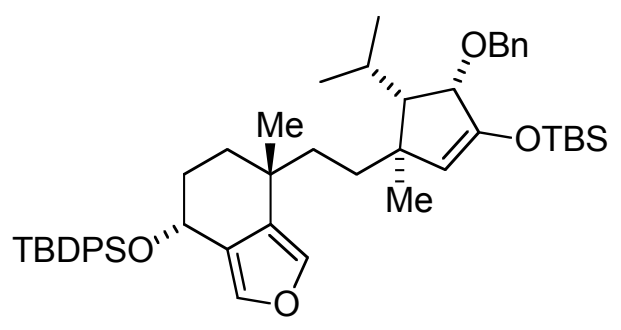

Silyl enol ether 32: A mixture of $31(99.4 \mathrm{mg}, 0.15 \mathrm{mmol})$ and 18-Crown-6 (63.4 $\mathrm{mg}, 0.24 \mathrm{mmol})$ was dissolved in benzene $(10 \mathrm{~mL})$, concentrated and then placed under high vacuum (200 mTorr) for $20 \mathrm{~min}$. It was then dissolved in THF $(3.0 \mathrm{~mL})$ and cooled to $-78{ }^{\circ} \mathrm{C}$ under argon. To this solution was added a solution of KHMDS (38.3 mg, $0.19 \mathrm{mmol})$ in THF $(1.0 \mathrm{~mL}+$ two $1.0 \mathrm{~mL}$ rinses $)$ via cannula dropwise. After $20 \mathrm{~min}$, TBSOTf ( $40 \mu \mathrm{l}, 0.17 \mathrm{mmol})$ was added. After $2 \mathrm{~h}$, the reaction was quenched with half saturated brine $(60 \mathrm{~mL})$ and diluted with $\mathrm{Et}_{2} \mathrm{O}(30 \mathrm{~mL})$. The two layers were separated and the aqueous layer was extracted with $\mathrm{Et}_{2} \mathrm{O}(2$ x $30 \mathrm{~mL}$ ). The combined organic extracts were dried, filtered and concentrated. The product was purified by column chromatography $\left(1 \rightarrow 2 \% \mathrm{Et}_{2} \mathrm{O}\right.$ in hexanes) to give $110 \mathrm{mg}$ ( $94 \%$ yield) of 32 as a white foam that collapsed to a sticky glass: $\mathrm{R}_{f} 0.25$ (3\% EtOAc in hexanes); $[\alpha]_{\mathrm{D}}-38.4^{\circ}\left(c=1.00, \mathrm{CHCl}_{3}\right)$; IR (thin film): $\tilde{v}=$ 2955, 2930, 2858, 1648, 1462, $1347 \mathrm{~cm}^{-1}$; ${ }^{1} \mathrm{H}$ NMR (500 MHz): $\delta=7.77$ (m, $\left.2 \mathrm{H}\right), 7.72$ (m, $\left.2 \mathrm{H}\right), 7.51-7.27$ (m, $11 \mathrm{H}), 7.14(\mathrm{~d}, J=1.5 \mathrm{~Hz}, 1 \mathrm{H}), 6.75(\mathrm{~s}, 1 \mathrm{H}), 5.02(\mathrm{~d}, J=11.9 \mathrm{~Hz}, 1 \mathrm{H}), 4.88(\mathrm{~m}, 1 \mathrm{H}), 4.68(\mathrm{~s}, 1 \mathrm{H}), 4.55(\mathrm{~d}, J$ $=11.9 \mathrm{~Hz}, 1 \mathrm{H}), 4.03(\mathrm{~d}, J=6.1 \mathrm{~Hz}, 1 \mathrm{H}), 2.20-2.10(\mathrm{~m}, 2 \mathrm{H}), 1.98-1.88(\mathrm{~m}, 1 \mathrm{H}), 1.82-1.25(\mathrm{~m}, 7 \mathrm{H}), 1.22(\mathrm{~s}, 3$ $\mathrm{H}), 1.16(\mathrm{~s}, 3 \mathrm{H}), 1.12(\mathrm{~s}, 9 \mathrm{H}), 1.05(\mathrm{~d}, J=6.5 \mathrm{~Hz}, 3 \mathrm{H}), 1.02-1.01(\mathrm{~m}, 12 \mathrm{H}), 0.28(\mathrm{~s}, 3 \mathrm{H}), 0.27(\mathrm{~s}, 3 \mathrm{H}) ;{ }^{13} \mathrm{C}$ NMR (100 MHz): $\delta=154.3,140.1,139.2,137.1,136.2,136.1,134.7,134.4,130.8,129.8,129.8,128.3,127.8$, 127.7, 127.1, 127.1, 125.0, 117.7, 85.0, 72.7, 63.6, 54.4, 46.7, 38.8, 35.6, 32.8, 31.1, 30.3, 30.2 27.2, 26.0, 25.5, 25.2, 22.9, 22.4, 19.5, 18.3, -4.4, -4.4; $\mathrm{HRMS}\left(\mathrm{FAB}^{+}\right)$: calcd for $\mathrm{C}_{49} \mathrm{H}_{67} \mathrm{O}_{4} \mathrm{Si}_{2}(\mathrm{M}-\mathrm{H})^{+}$: 775.4578; found: 775.4580 . 


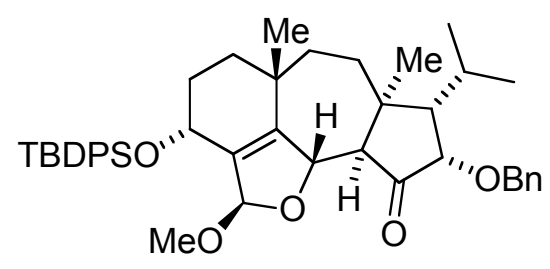

Electrochemistry product 36: To a $100 \mathrm{~mL}$ 3-neck round-bottomed flask was added silyl enol ether 32 (165 $\mathrm{mg}, 0.21 \mathrm{mmol})$, lithium perchlorate (425 mg, $4.0 \mathrm{mmol}), 2$,6-lutidine $(0.15 \mathrm{~mL}, 1.29 \mathrm{mmol}), \mathrm{MeOH}(8 \mathrm{~mL})$, and $\mathrm{CH}_{2} \mathrm{Cl}_{2}(32 \mathrm{~mL})$. The reaction flask was then fitted with a $\mathrm{RVC}$ anode $\left(\mathrm{ca} .1 \mathrm{~cm}^{3}\right)$ and a platinum cathode and sonicated under argon for $5 \mathrm{~min}$. A constant current of $0.9 \mathrm{~mA}$ was applied to the solution until a total of $53.5 \mathrm{C}(2.61 \mathrm{~F} / \mathrm{mol})$ of charge had been passed $(16.5 \mathrm{~h})$. The reaction was deemed complete by thin layer chromatography, and the mixture was diluted with $\mathrm{Et}_{2} \mathrm{O}(200 \mathrm{~mL})$ and half saturated brine $(200 \mathrm{~mL})$. The two layers were separated and the aqueous layer was extracted with $\mathrm{Et}_{2} \mathrm{O}(2 \times 50 \mathrm{~mL})$. The combined organic extracts were dried and concentrated. The product was purified by column chromatography $(12 \%$ EtOAc in hexanes) to afford $119 \mathrm{mg}(81 \%)$ of $\mathbf{3 6}$ as a foamy solid: $\mathrm{R}_{f} 0.39(20 \%$ EtOAc in hexanes $) ;[\alpha]_{\mathrm{D}}-63.9^{\circ}(c=$ 1.00, $\mathrm{CHCl}_{3}$ ); $\mathrm{mp} \mathrm{50-52}{ }^{\circ} \mathrm{C}$; IR (thin film): $\tilde{v}=2955,2931,2857,1745 \mathrm{~cm}^{-1} ;{ }^{1} \mathrm{H}$ NMR $\left(500 \mathrm{MHz}, 53{ }^{\circ} \mathrm{C}\right): \delta=$ $7.73(\mathrm{~m}, 4 \mathrm{H}), 7.47-7.28(\mathrm{~m}, 11 \mathrm{H}), 5.60(\mathrm{~s}, 1 \mathrm{H}), 5.15(\mathrm{~d}, J=11.2 \mathrm{~Hz}, 1 \mathrm{H}), 5.10$ (br s, $1 \mathrm{H}), 4.55$ (d, $J=11.4$ Hz, 1 H), 4.46 (s, 1 H), 3.89 (d, J=7.2 Hz, 1 H), 2.36-2.25 (m, 2 H), 1.92-1.67 (m, 6 H), 1.56 (app t, 1 H), $1.43-$ $1.32(\mathrm{~m}, 2 \mathrm{H}), 1.27(\mathrm{~s}, 3 \mathrm{H}), 1.13(\mathrm{~s}, 9 \mathrm{H}), 1.10(\mathrm{~d}, J=6.6 \mathrm{~Hz}, 3 \mathrm{H}), 1.06-1.03(\mathrm{~m}, 6 \mathrm{H}),{ }^{13} \mathrm{C} \mathrm{NMR}(125 \mathrm{MHz}, 53$ $\left.{ }^{\circ} \mathrm{C}\right): \delta=214.6,149.1,138.5,136.0,135.9,134.6,134.4,132.6,129.8,129.8,128.4,127.9,127.8,127.7,127.6$, $108.1,80.1,79.8,73.3,65.4,62.3,57.2,54.4,43.6,35.5,34.7,34.6,34.0,28.8,27.2,25.2,24.9,23.9,23.5,23.2$, 19.6; HRMS $\left(\mathrm{FAB}^{+}\right)$: calcd for $\mathrm{C}_{44} \mathrm{H}_{56} \mathrm{O}_{5} \mathrm{SiLi}(\mathrm{M}+\mathrm{Li})^{+}$: 699.4057; found: 699.4039 .

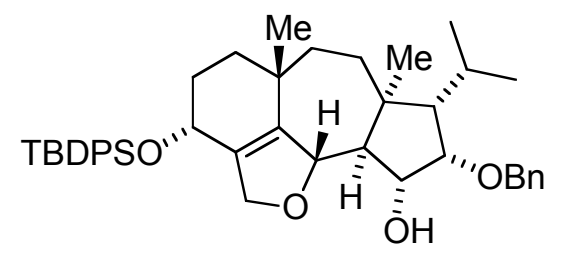

Secondary alcohol 37: To a solution of acetal $36(50.0 \mathrm{mg}, 0.072 \mathrm{mmol})$ in PhMe $(5.0 \mathrm{~mL})$ was added a solution of DIBAL $(0.72 \mathrm{~mL}, 0.72 \mathrm{mmol}, 1.0 \mathrm{M}$ in $\mathrm{PhMe})$ at $-78{ }^{\circ} \mathrm{C}$ under argon. After $5 \mathrm{~min}$, the reaction was allowed to warm to rt. After $2 \mathrm{~h}$, the reaction was added into half saturated aqueous Rochelle's salt (200 mL) and diluted with EtOAc $(100 \mathrm{~mL})$. The two layers were separated and the aqueous layer was extracted with EtOAc $(2 \times 50 \mathrm{~mL})$. The combined organic extracts were dried, filtered, and concentrated. The product was purified by column chromatography (10\% EtOAc in hexanes) to provide $29.5 \mathrm{mg}$ (61\% yield) of $\mathbf{3 7}$ as a white foam: $\mathrm{R}_{f}$ $0.43\left(20 \%\right.$ EtOAc in hexanes); $[\alpha]_{\mathrm{D}}+50.4^{\circ}\left(c=1.35, \mathrm{CHCl}_{3}\right) ; \mathrm{mp} 48-49^{\circ} \mathrm{C}$; IR (thin film): $\tilde{v}=3516(\mathrm{br}), 2956$, 2900, $2856 \mathrm{~cm}^{-1} ;{ }^{1} \mathrm{H}$ NMR (500 MHz): $\delta=7.65(\mathrm{~m}, 4 \mathrm{H}), 7.45-7.27(\mathrm{~m}, 11 \mathrm{H}), 5.28(\mathrm{~d} J=12.0 \mathrm{~Hz}, 1 \mathrm{H}), 4.78$ $(\mathrm{dd}, J=9.1,4.3 \mathrm{~Hz}, 1 \mathrm{H}), 4.52(\mathrm{~d}, J=12.0 \mathrm{~Hz}, 1 \mathrm{H}), 4.39(\mathrm{dd}, J=11.9,4.5 \mathrm{~Hz}, 1 \mathrm{H}), 4.18(\mathrm{br} \mathrm{s}, 1 \mathrm{H}), 4.01(\mathrm{dd}$, 
$J=9.6,2.9 \mathrm{~Hz}, 1 \mathrm{H}), 3.95$ (app t, $1 \mathrm{H}), 3.84$ (d, $J=11.9 \mathrm{~Hz}, 1 \mathrm{H}), 2.22-2.14$ (m, $1 \mathrm{H}), 1.92-1.52$ (m, $8 \mathrm{H}), 1.42-$ $1.27(\mathrm{~m}, 6 \mathrm{H}), 1.18(\mathrm{dd}, J=10.2,3.3 \mathrm{~Hz}, 1 \mathrm{H}), 1.05(\mathrm{~s}, 9 \mathrm{H}), 1.00(\mathrm{~s}, 3 \mathrm{H}), 0.98(\mathrm{~d}, J=6.6 \mathrm{~Hz}, 3 \mathrm{H}), 0.94(\mathrm{~d}, J=$ $6.5 \mathrm{~Hz}, 3 \mathrm{H}) ;{ }^{13} \mathrm{C}$ NMR (125 MHz): $\delta=144.2,140.6,136.1,135.9,134.2,134.0,133.4,130.0,129.9,128.3$, $127.9,127.8,127.0,126.9,89.4,82.7,82.5,74.0,74.0,64.2,59.2,58.2,42.4,36.4,35.0,34.1,33.7,29.7,27.1$, 26.1, 25.4, 25.1, 23.8, 23.5, 19.6; HRMS $\left(\mathrm{FAB}^{+}\right)$: calcd for $\mathrm{C}_{43} \mathrm{H}_{55} \mathrm{O}_{4} \mathrm{Si}(\mathrm{M}-\mathrm{H})^{+}$: 663.3870; found: 663.3857 .

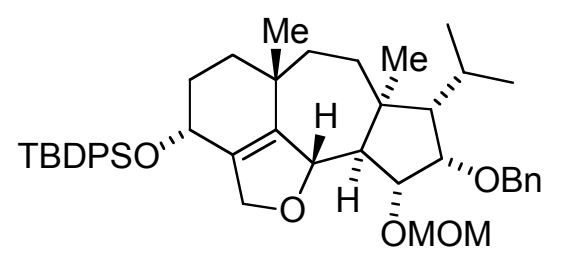

Protected triol S2: A solution of alcohol 37 (66.9 mg, $0.101 \mathrm{mmol})$, NaI (45.0 mg, $0.30 \mathrm{mmol}), i$-Pr ${ }_{2} \mathrm{NEt}(0.13$ $\mathrm{mL}, 0.75 \mathrm{mmol})$ and $\mathrm{MOMCl}(40 \mu \mathrm{L}, 0.53 \mathrm{mmol})$ in THF $(1.5 \mathrm{~mL})$ was heated to reflux under argon for $20 \mathrm{~h}$. The reaction mixture was diluted with $\mathrm{H}_{2} \mathrm{O}(4 \mathrm{~mL})$ and EtOAc $(2 \mathrm{~mL})$. The two layers were separated and the aqueous layer was extracted with EtOAc $(2 \times 2 \mathrm{~mL})$. The combined organic extracts were dried and concentrated. The product was purified by column chromatography (10\% EtOAc in hexanes) to give $67.5 \mathrm{mg}$ (95\% yield) of $\mathbf{S} 2$ as a white foam: $\mathrm{R}_{f} 0.38\left(20 \%\right.$ EtOAc in hexanes); $[\alpha]_{\mathrm{D}}+28.0^{\circ}\left(c=3.33, \mathrm{CHCl}_{3}\right) ; \mathrm{mp} 48-49$ ${ }^{\circ} \mathrm{C}$; IR (thin film): $\tilde{v}=2970,2930,1462 \mathrm{~cm}^{-1} ;{ }^{1} \mathrm{H}$ NMR (500 MHz): $\delta=7.67-7.64(\mathrm{~m}, 4 \mathrm{H}), 7.43-7.26(\mathrm{~m}, 11$ H), $5.20(\mathrm{~d}, J=11.5 \mathrm{~Hz}, 1 \mathrm{H}), 4.86(\mathrm{~d}, J=6.8 \mathrm{~Hz}, 1 \mathrm{H}), 4.80(\mathrm{~d}, J=6.8 \mathrm{~Hz}, 1 \mathrm{H}), 4.75(\mathrm{~m}, 1 \mathrm{H}), 4.62(\mathrm{dd}, J=$ 12.3, $4.5 \mathrm{~Hz}, 1 \mathrm{H}), 4.39$ (d, $J=11.5 \mathrm{~Hz}, 1 \mathrm{H}), 4.23(\mathrm{~m}, 1 \mathrm{H}), 3.99-3.91(\mathrm{~m}, 3 \mathrm{H}), 3.48(\mathrm{~s}, 3 \mathrm{H}), 2.24(\mathrm{dd}, J=10.4$, $7.6 \mathrm{~Hz}, 1 \mathrm{H}), 2.13$ (m, 1 H), 1.86-1.79 (m, $1 \mathrm{H}), 1.76-1.66$ (m, 3 H), 1.61-1.58 (m, $2 \mathrm{H}), 1.35-1.28(\mathrm{~m}, 1 \mathrm{H}), 1.27$ (s, 3 H), 1.24-1.18 (m, 1 H), 1.08 (dd, $J=10.4,3.4 \mathrm{~Hz}, 1 \mathrm{H}), 1.04$ (s, $9 \mathrm{H}), 1.00$ (s, $3 \mathrm{H}), 0.97$ (d, $J=6.5 \mathrm{~Hz}, 3$ $\mathrm{H}), 0.94(\mathrm{~d}, J=6.5 \mathrm{~Hz}, 3 \mathrm{H}) ;{ }^{13} \mathrm{C}$ NMR $(125 \mathrm{MHz}): \delta=143.1,140.3,136.2,136.0,135.1$, 134.3, 134.1, 129.8, 129.8, 128.3, 127.8, 127.7, 127.1, 127.0, 95.9, 87.1, 84.6, 80.6, 73.7, 73.7, 65.7, 58.0, 56.7, 55.9, 41.5, 35.9,

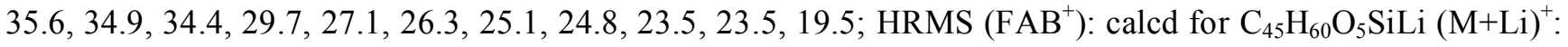
715.4363; found: 715.4357 .

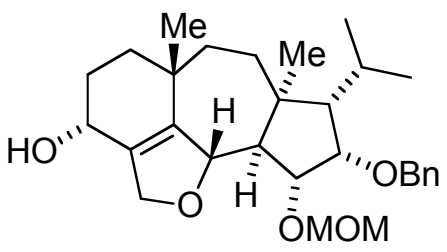

Secondary alcohol S3: To a solution of S2 $(64.0 \mathrm{mg}, 0.090 \mathrm{mmol})$ in THF $(0.2 \mathrm{~mL})$ was added a solution of TBAF (0.5 mL, $0.5 \mathrm{mmol}, 1.0 \mathrm{M}$ in THF) at $\mathrm{rt}$ under $\mathrm{N}_{2}$. The vial was capped and stirred for $5 \mathrm{~h}$ at which time the reaction mixture was diluted with half saturated brine $(4 \mathrm{~mL})$ and EtOAc $(2 \mathrm{~mL})$. The two layers were separated and the aqueous layer was extracted with EtOAc $(3 \times 2 \mathrm{~mL})$. The combined organic extracts were dried, filtered and concentrated. The product was purified by column chromatography $(40 \rightarrow 50 \%$ EtOAc in 
hexanes) to give (42.5 mg, 100\% yield) of $\mathbf{S 3}$ as a white foam: $\mathrm{R}_{f} 0.24\left(50 \%\right.$ EtOAc in hexanes); $[\alpha]_{\mathrm{D}}+11.4^{\circ}(c$ $\left.=2.15, \mathrm{CHCl}_{3}\right) ; \mathrm{mp} 43-44{ }^{\circ} \mathrm{C}$; IR (thin film): $\tilde{v}=3416$ (br), 2930, $1452 \mathrm{~cm}^{-1} ;{ }^{1} \mathrm{H}$ NMR $(500 \mathrm{MHz}): \delta=7.35-$ $7.28(\mathrm{~m}, 4 \mathrm{H}), 7.25-7.21(\mathrm{~m}, 1 \mathrm{H}), 5.15(\mathrm{~d}, J=11.5 \mathrm{~Hz}, 1 \mathrm{H}), 4.85(\mathrm{~d}, J=6.8 \mathrm{~Hz}, 1 \mathrm{H}), 4.83-4.77$ (m, 3 H), 4.40 $(\mathrm{d}, J=11.3 \mathrm{~Hz}, 1 \mathrm{H}), 4.36(\mathrm{~d}, J=11.5 \mathrm{~Hz}, 1 \mathrm{H}), 4.22(\operatorname{app~t}, 1 \mathrm{H}), 3.99(\mathrm{dd}, J=10.2,3.1 \mathrm{~Hz}, 1 \mathrm{H}), 3.92$ (app t, 1 H) $3.49(\mathrm{~s}, 3 \mathrm{H}), 2.18(\mathrm{dd}, J=9.8,7.7 \mathrm{~Hz}, 1 \mathrm{H}), 2.08(\mathrm{~m}, 1 \mathrm{H}), 1.94-1.87(\mathrm{~m}, 1 \mathrm{H}), 1.78-1.55(\mathrm{~m}, 5 \mathrm{H}), 1.46-1.40$ $(\mathrm{m} 1 \mathrm{H}), 1.32-1.19(\mathrm{~m}, 2 \mathrm{H}), 1.15(\mathrm{~s}, 3 \mathrm{H}), 1.07$ (dd, $J=10.3,3.7 \mathrm{~Hz}, 1 \mathrm{H}), 1.05(\mathrm{~s}, 3 \mathrm{H}), 0.94(\mathrm{~d}, J=6.5 \mathrm{~Hz}, 3$ H), $0.90(\mathrm{~d}, J=6.6 \mathrm{~Hz}, 3 \mathrm{H}) ;{ }^{13} \mathrm{C}$ NMR $(125 \mathrm{MHz}): \delta=144.1,140.1,134.4,128.2,127.0,126.9$, 95.9, 87.1, 84.3, 80.6, 73.7, 73.3, 64.4, 58.0, 57.3, 56.0, 41.8, 35.7, 35.4, 34.7, 34.5, 29.7, 26.2, 25.0, 25.0, 23.5, 23.5; HRMS $\left(\mathrm{FAB}^{+}\right)$: calcd for $\mathrm{C}_{29} \mathrm{H}_{42} \mathrm{O}_{5} \mathrm{Li}(\mathrm{M}+\mathrm{Li})^{+}$: 477.3192; found: 477.3206.

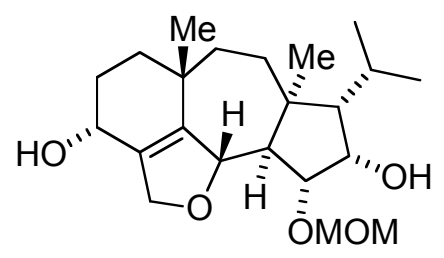

Diol 38: To a solution of secondary alcohol S3 $(41.0 \mathrm{mg}, 0.087 \mathrm{mmol})$ in THF $(2.0 \mathrm{~mL})$ was condensed liquid $\mathrm{NH}_{3}(\sim 3 \mathrm{~mL})$ at $-78^{\circ} \mathrm{C}$ under $\mathrm{N}_{2}$. To this mixture was added sodium metal (16 mg, 2 chunks). After 2-3 min, the blue color persisted and the reaction mixture was stirred at $-78^{\circ} \mathrm{C}$ for $1.5 \mathrm{~h}$ at which time it was quenched with solid $\mathrm{NH}_{4} \mathrm{Cl}$ (excess) and was allowed to warm to rt. The mixture was diluted with EtOAc $(5 \mathrm{~mL})$ and $\mathrm{H}_{2} \mathrm{O}$ $(10 \mathrm{~mL})$. The two layers were separated and the aqueous layer was extracted with EtOAc $(3 \times 3 \mathrm{~mL})$. The combined organic extracts were dried, filtered and concentrated. The product was purified by column chromatography $\left(7.5 \% \mathrm{MeOH}\right.$ in $\left.\mathrm{CH}_{2} \mathrm{Cl}_{2}\right)$ to give $31 \mathrm{mg}\left(93.5 \%\right.$ yield) of $\mathbf{3 8}$ as a white foam: $\mathrm{R}_{f} 0.31(8 \%$ $\mathrm{MeOH}$ in $\left.\mathrm{CH}_{2} \mathrm{Cl}_{2}\right) ;[\alpha]_{\mathrm{D}}-26.4^{\circ}\left(c=1.50, \mathrm{CHCl}_{3}\right)$; mp $40-41^{\circ} \mathrm{C}$; IR (thin film): $\tilde{v}=3434$ (br), $2931,1460 \mathrm{~cm}^{-1}$; ${ }^{1} \mathrm{H}$ NMR $(500 \mathrm{MHz}): \delta=4.81(\mathrm{~d}, J=6.6 \mathrm{~Hz}, 1 \mathrm{H}), 4.74(\mathrm{dd}, J=11.9,5.0 \mathrm{~Hz}, 1 \mathrm{H}), 4.71(\mathrm{~d}, J=6.6 \mathrm{~Hz}, 1 \mathrm{H})$, $4.69(\mathrm{~m}, 1 \mathrm{H}), 4.37(\mathrm{~d}, J=11.9 \mathrm{~Hz}, 1 \mathrm{H}), 4.19(\mathrm{~m}, 1 \mathrm{H}), 4.15($ app t, $1 \mathrm{H}), 3.99$ (dd, J = 7.6, $4.7 \mathrm{~Hz}, 1 \mathrm{H}), 3.42$ (s, $3 \mathrm{H}), 2.47$ (s, $1 \mathrm{H}), 2.08$ (m, $1 \mathrm{H}), 1.96-1.87$ (m, $2 \mathrm{H}), 1.74-1.49(\mathrm{~m}, 6 \mathrm{H}), 1.42(\mathrm{ddd}, J=13.4,6.7,2.9 \mathrm{~Hz}, 1 \mathrm{H})$, 1.03-1.23 (m, $1 \mathrm{H}), 1.16(\mathrm{~s}, 3 \mathrm{H}), 1.07(\mathrm{dd}, J=10.2,3.9 \mathrm{~Hz}, 1 \mathrm{H}), 1.05(\mathrm{~d}, J=6.5 \mathrm{~Hz}, 3 \mathrm{H}), 1.02(\mathrm{~s}, 3 \mathrm{H}), 0.96$ $(\mathrm{d}, J=6.6 \mathrm{~Hz}, 3 \mathrm{H}) ;{ }^{13} \mathrm{C}$ NMR $(125 \mathrm{MHz}): \delta=144.7,133.4,96.4,86.7,83.6,73.2,73.2,63.6,59.4,59.0,56.0$, 43.2, 35.6, 35.1, 34.3, 34.3, 29.4, 25.3, 25.2, 25.1, 23.7, 23.6; HRMS $\left(\mathrm{FAB}^{+}\right)$: calcd for $\mathrm{C}_{22} \mathrm{H}_{36} \mathrm{O}_{5} \mathrm{Li}(\mathrm{M}+\mathrm{Li})^{+}$: 387.2728; found: 387.2730 .

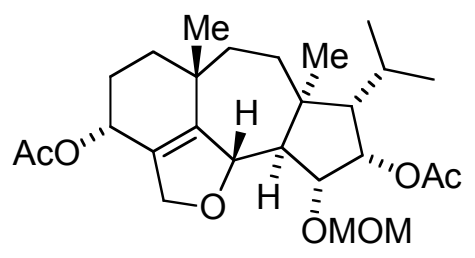


Diacetate S4: To a solution of diol $38(23 \mathrm{mg}, 0.060 \mathrm{mmol})$ and DMAP (2.2 mg, $0.018 \mathrm{mmol})$ in $p$-xylene $(0.5$ $\mathrm{mL})$ was added pyridine $(30 \mu \mathrm{L}, 0.37 \mathrm{mmol})$ and $\mathrm{Ac}_{2} \mathrm{O}(60 \mu \mathrm{L}, 0.64 \mathrm{mmol})$. The mixture was placed under argon, sealed in a vial and heated to $135^{\circ} \mathrm{C}$. After $12 \mathrm{~h}$, the reaction mixture was concentrated (200 mTorr) and purified by column chromatography (35\% EtOAc in hexanes) to give $26.0 \mathrm{mg}$ (93\% yield) of $\mathbf{S 4}$ as a foam that collapsed to a glass: $\mathrm{R}_{f} 0.38\left(50 \%\right.$ EtOAc in hexanes); $[\alpha]_{\mathrm{D}}+57.2^{\circ}\left(c=1.00, \mathrm{CHCl}_{3}\right)$; IR (thin film): $\tilde{v}=2957$, 1736, 1450, 1372, $1244 \mathrm{~cm}^{-1} ;{ }^{1} \mathrm{H}$ NMR (500 MHz): $\delta=5.39(\operatorname{app~t,} 1 \mathrm{H}), 5.25(\mathrm{~m}, 1 \mathrm{H}), 4.78-4.72(\mathrm{~m}, 2 \mathrm{H})$, $4.59(\mathrm{dd}, J=12.4,4.9 \mathrm{~Hz}, 1 \mathrm{H}), 4.55(\mathrm{~d}, J=7.0 \mathrm{~Hz}, 1 \mathrm{H}), 4.34$ (d, $J=12.4 \mathrm{~Hz}, 1 \mathrm{H}), 4.00$ (dd, $J=9.6,4.9 \mathrm{~Hz}, 1$ H), $3.40(\mathrm{~s}, 3 \mathrm{H}), 2.07(\mathrm{~s}, 3 \mathrm{H}), 2.02(\mathrm{~s}, 3 \mathrm{H}), 2.00-1.57(\mathrm{~m}, 8 \mathrm{H}), 1.45$ (ddd, J = 13.2, 7.0, $2.9 \mathrm{~Hz}, 1 \mathrm{H}), 1.32-$ $1.20(\mathrm{~m}, 2 \mathrm{H}), 1.15(\mathrm{~s}, 3 \mathrm{H}), 1.05(\mathrm{~s}, 3 \mathrm{H}), 0.95(\mathrm{~d}, J=6.5 \mathrm{~Hz}, 3 \mathrm{H}), 0.84(\mathrm{~d}, J=6.5 \mathrm{~Hz}, 3 \mathrm{H}) ;{ }^{13} \mathrm{C}$ NMR $(125$ MHz): $\delta=171.1,170.8,146.4,131.0,95.6,86.7,80.2,74.2,73.5,66.0,58.3,56.7,56.0,42.5,35.6,35.5,34.6$, 34.4, 25.9, 25.6, 25.1, 24.7, 23.5, 23.1, 21.4, 21.4; HRMS $\left(\mathrm{FAB}^{+}\right)$: calcd for $\mathrm{C}_{26} \mathrm{H}_{40} \mathrm{O}_{7} \mathrm{Li}(\mathrm{M}+\mathrm{Li})^{+}$: 471.2934; found: 471.2937 .

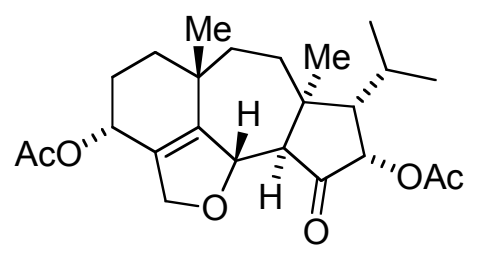

(-)-heptemerone B: To a solution of diacetate $\mathbf{S 4}(22.0 \mathrm{mg}, 0.047 \mathrm{mmol})$ in $\mathrm{CH}_{2} \mathrm{Cl}_{2}(2.0 \mathrm{~mL})$ and DMS $(1.0 \mathrm{~mL})$ was added $\mathrm{BF}_{3} \cdot \mathrm{OEt}_{2}(90 \mu \mathrm{L})$ at $-20{ }^{\circ} \mathrm{C}$ under argon. After $4 \mathrm{~h}$, the reaction mixture was quenched with half saturated aqueous $\mathrm{NaHCO}_{3}(5 \mathrm{~mL})$ and diluted with EtOAc $(3 \mathrm{~mL})$. The two layers were separated and the aqueous layer was extracted with EtOAc $(3 \times 2 \mathrm{~mL})$. The combined organic extracts were dried, filtered and concentrated. This material was taken on directly to the next step without further purification or characterization because of its propensity to undergo 1,2-acyl migration. To a solution of the crude secondary alcohol in $\mathrm{CH}_{2} \mathrm{Cl}_{2}$ out of the squirt bottle $(1.0 \mathrm{~mL})$ was added Dess-Martin periodinane $(30.0 \mathrm{mg}, 0.071 \mathrm{mmol})$ at $\mathrm{rt}$ open to the air. After $2 \mathrm{~h}$, the reaction mixture was quenched with a 1:1:1 mixture of saturated aqueous $\mathrm{NaHCO}_{3}-$ saturated aqueous $\mathrm{Na}_{2} \mathrm{~S}_{2} \mathrm{O}_{3}-\mathrm{H}_{2} \mathrm{O}(3 \mathrm{~mL})$ and diluted with EtOAc $(2 \mathrm{~mL})$. The two layers were separated and the aqueous layer was extracted with EtOAc $(3 \times 2 \mathrm{~mL})$. The combined organic extracts were dried, filtered and concentrated. The product was purified by column chromatography (28\% EtOAc in hexanes) to give $13.6 \mathrm{mg}$ ( $69 \%$ for 2 steps) of (-)-heptemerone B as a white solid: $\mathrm{R}_{f} 0.37$ (40\% EtOAc in hexanes); $[\alpha]_{\mathrm{D}}-116^{\circ}\left(c=0.36, \mathrm{CHCl}_{3}\right)$; IR (thin film): $\tilde{v}=2959,2935,1758,1736,1371,1229,1055,1019 \mathrm{~cm}^{-1}$; HRMS $\left(\mathrm{FAB}^{+}\right)$: calcd for $\mathrm{C}_{24} \mathrm{H}_{35} \mathrm{O}_{6}(\mathrm{M}+\mathrm{H})^{+}$: 419.2434; found: 419.2437 . 
Table S1: Comparison of NMR data for heptemerone $B^{4}$

\begin{tabular}{|c|c|c|c|}
\hline${ }^{1} \mathrm{H}$ (lit.) & ${ }^{1} \mathrm{H}$ (current $\left.500 \mathrm{MHz}\right)$ & ${ }^{13} \mathrm{C}$ (lit.) & ${ }^{13} \mathrm{C}$ (current $\left.125 \mathrm{MHz}\right)$ \\
\hline 0.90 & 0.89 & 20.9 & 21.0 \\
\hline 1.03 & 1.01 & 21.4 & 22.3 \\
\hline 1.06 & 1.05 & 22.3 & 22.9 \\
\hline 1.07 & 1.06 & 22.9 & 23.0 \\
\hline 1.43 & 1.42 & 23.0 & 23.5 \\
\hline 1.43 & 1.42 & 23.5 & 25.7 \\
\hline 1.43 & 1.42 & 25.7 & 25.8 \\
\hline 1.57 & 1.56 & 25.8 & 33.9 \\
\hline 1.81 & 1.81 & 33.7 & 34.0 \\
\hline 1.86 & 1.85 & 34.0 & 34.5 \\
\hline 1.92 & 1.91 & 34.5 & 35.9 \\
\hline 1.98 & 1.98 & 35.9 & 44.0 \\
\hline 2.00 & 2.00 & 44.0 & 56.1 \\
\hline 2.04 & 2.04 & 56.1 & 63.8 \\
\hline 2.14 & 2.13 & 63.8 & 66.6 \\
\hline 2.14 & 2.14 & 66.6 & 73.5 \\
\hline 2.20 & 2.19 & 73.4 & 74.9 \\
\hline 4.43 & 4.42 & 74.9 & 80.8 \\
\hline 4.59 & 4.58 & 80.8 & 128.4 \\
\hline 4.99 & 4.98 & 128.4 & 147.3 \\
\hline 5.27 & 5.26 & 147.2 & 169.9 \\
\hline 5.68 & 5.67 & 169.9 & 171.0 \\
\hline & & 170.9 & 210.8 \\
\hline & & 210.6 & \\
\hline & & & \\
\hline & & & \\
\hline
\end{tabular}

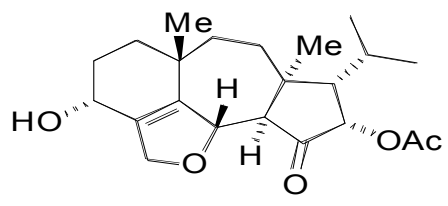

(-)-guanacastepene $\mathbf{E}$ : To a solution of (-)-heptemerone B $(6.8 \mathrm{mg}, 0.016 \mathrm{mmol})$ in $\mathrm{MeOH}(0.70 \mathrm{~mL})$ was added anhydrous $\mathrm{K}_{2} \mathrm{CO}_{3}(2.4 \mathrm{mg}, 0.017 \mathrm{mmol})$ at $0{ }^{\circ} \mathrm{C}$ under argon. After the addition, the reaction mixture was allowed to warm to rt. After $45 \mathrm{~min}$, the reaction mixture was quenched with saturated aqueous $\mathrm{NH}_{4} \mathrm{Cl}(4 \mathrm{~mL})$ and diluted with EtOAc $(2 \mathrm{~mL})$. The two layers were separated and the aqueous layer was extracted with EtOAc $(3 \times 2 \mathrm{~mL})$. The combined organic extracts were dried, filtered and concentrated. The product was purified by column chromatography (45\% EtOAc in hexanes) to give $1.7 \mathrm{mg}(28 \%)$ of guanacastepene $\mathrm{E}$ as a white solid along with $2.0 \mathrm{mg}$ of recovered heptemerone B. Data for (-)-guanacastepene $\mathrm{E}: \mathrm{R}_{f} 0.17$ (50\% EtOAc in hexanes); $[\alpha]_{\mathrm{D}}-134.6^{\circ}\left(c=0.227, \mathrm{CHCl}_{3}\right)$; IR (thin film): $\tilde{v}=3412,2931,2873,1756,1455,1371,1226,1055$, $1028 \mathrm{~cm}^{-1} ;{ }^{1} \mathrm{H}$ NMR (500 MHz in acetone-d6 calibrated to $\left.2.05 \mathrm{ppm}\right): \delta=5.60(\mathrm{~d}, J=9.5 \mathrm{~Hz}, 1 \mathrm{H}), 5.10(\mathrm{~m}, 1$ H), $4.66(\mathrm{dd}, J=11.8,4.9 \mathrm{~Hz}, 1 \mathrm{H}), 4.33(\mathrm{~d}, J=11.8 \mathrm{~Hz}, 1 \mathrm{H}), 4.11(\mathrm{~m}, 1 \mathrm{H}), 3.86(\mathrm{~d}, J=7.0 \mathrm{~Hz}, 1 \mathrm{H}), 2.43(\mathrm{t}$, 
$J=10.0 \mathrm{~Hz}, 1 \mathrm{H}), 2.10-2.07$ (m, $4 \mathrm{H}), 2.00-1.88$ (m, $4 \mathrm{H}), 1.78-1.69$ (m, $2 \mathrm{H}), 1.45$ (app t, $2 \mathrm{H}), 1.35$ (m, $1 \mathrm{H})$, $1.09(\mathrm{~d}, J=6.7 \mathrm{~Hz}, 3 \mathrm{H}), 1.06(\mathrm{~s}, 3 \mathrm{H}), 0.98(\mathrm{~s}, 3 \mathrm{H}), 0.88(\mathrm{~d}, J=6.4 \mathrm{~Hz}, 3 \mathrm{H})$; HRMS $\left(\mathrm{FAB}^{+}\right)$: calcd for $\mathrm{C}_{22} \mathrm{H}_{33} \mathrm{O}_{5}(\mathrm{M}+\mathrm{H})^{+}:$377.2328; found: $377.2331 .{ }^{1} \mathrm{H}$ NMR data for guanacastepene $\mathrm{E}$ was not tabulated in the isolation paper. ${ }^{5}$ Copies of the original ${ }^{1} \mathrm{H}$ - and ${ }^{13} \mathrm{C}$ spectra are included below for comparison.

Table S2: Comparison of NMR data for guanacastepene E (acetone-d6) ${ }^{5}$

\begin{tabular}{|c|c|}
\hline${ }^{13} \mathrm{C}$ (lit.) & ${ }^{13} \mathrm{C}$ (current $125 \mathrm{MHz}$ ) \\
\hline 20.7 & 20.8 \\
\hline 22.4 & 22.4 \\
\hline 23.0 & 23.1 \\
\hline 23.3 & 23.3 \\
\hline 23.6 & 23.6 \\
\hline 26.4 & 26.5 \\
\hline 29.7 & $\sim 30$ (under solvent) \\
\hline 34.5 & 34.5 \\
\hline 34.6 & 34.5 \\
\hline 36.6 & 34.6 \\
\hline 44.5 & 36.6 \\
\hline 55.8 & 44.5 \\
\hline 61.3 & 55.8 \\
\hline 61.4 & 61.4 \\
\hline 67.6 & 67.6 \\
\hline 74.1 & 74.1 \\
\hline 75.2 & 75.2 \\
\hline 81.5 & 81.5 \\
\hline 133 & 132.9 \\
\hline 144.6 & 144.6 \\
\hline 170.2 & 170.2 \\
\hline 210.5 & 210.5 \\
\hline & \\
\hline
\end{tabular}

${ }_{1}^{1}$ Alaimo, P. J.; Peters, D. W.;Arnold, J.; Bergman, R. G. J. Chem. Ed. 2001, 78, 64.

${ }^{2}$ Heathcock, C. H.; Tice, C. M.; Germroth, T. C. J. Am. Chem. Soc. 1982, 104, 6081-6091.

${ }^{3}$ Still, W. C.; Kahn, M.; Mitra, A. J. Org. Chem. 1978, 43, 2923-2925.

${ }^{4}$ Valdivia, C.; Kettering, M.; Anke, H.; Thines, E.; Sterner, O. Tetrahedron 2005, 61, 9527-9532.

${ }^{5}$ Brady, S. F.; Bondi, S. M.; Clardy, J. J. Am. Chem. Soc. 2001, 123, 9900-9901. 

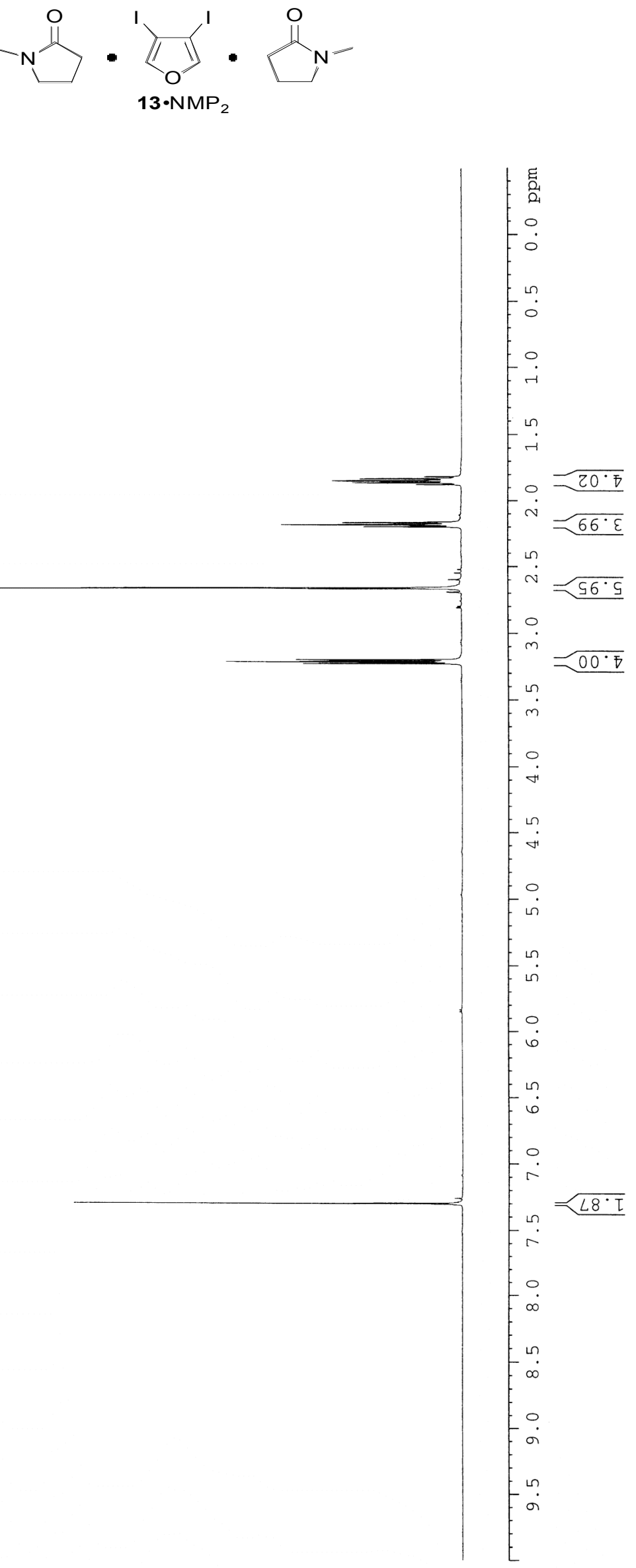


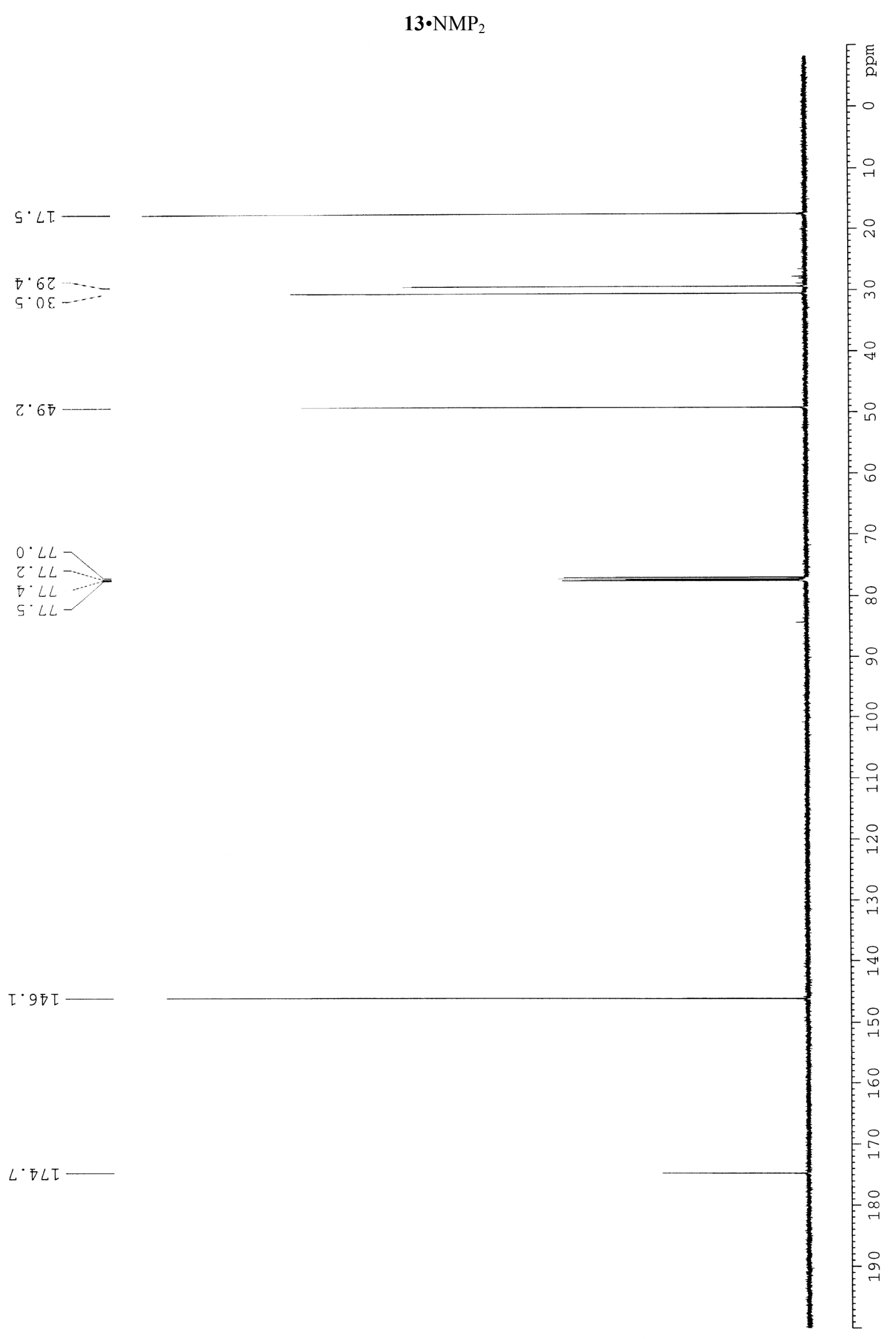




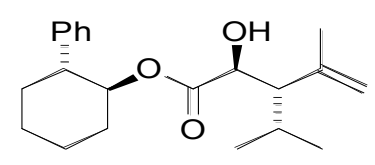

$( \pm)-26$

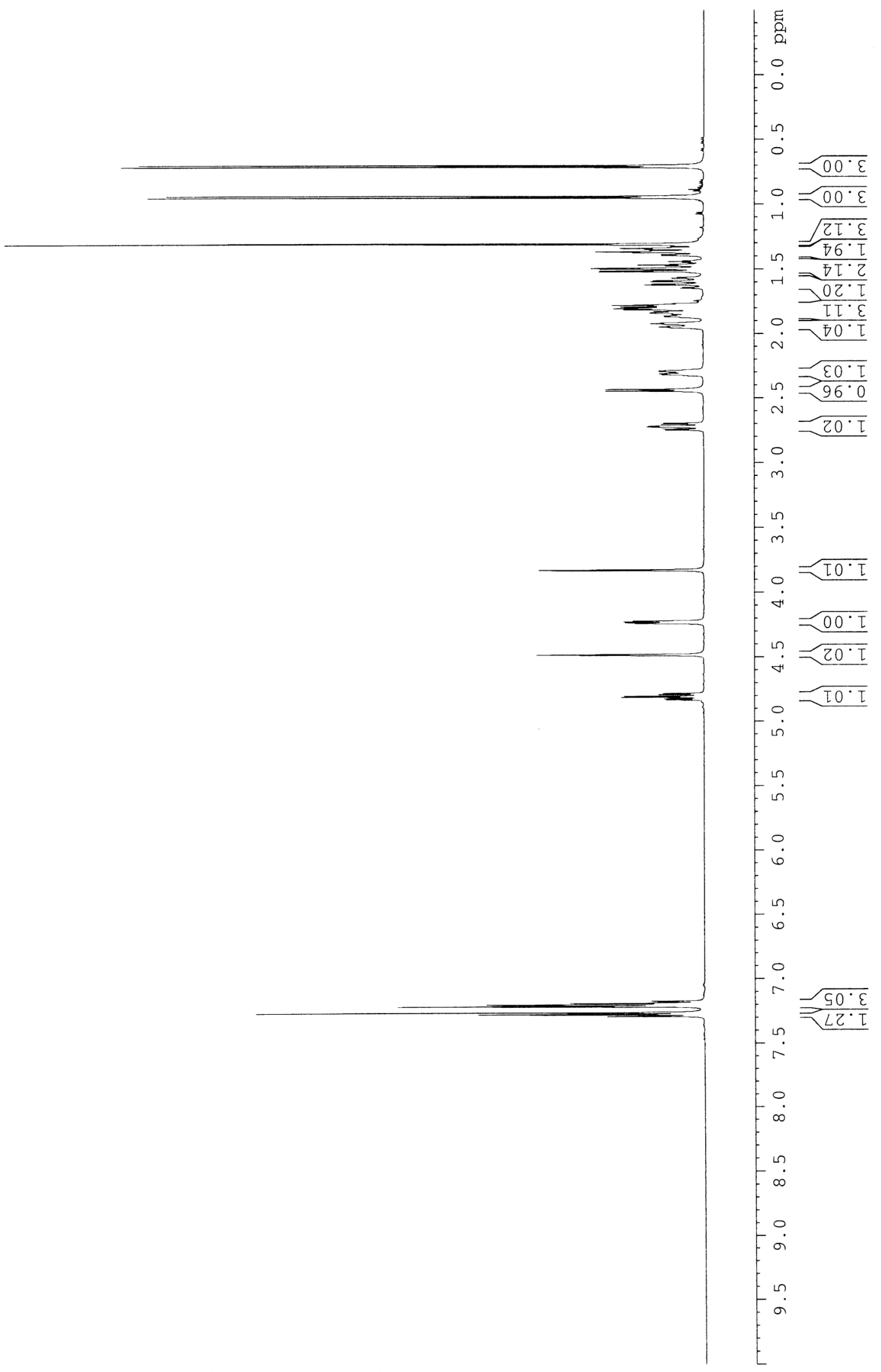


( \pm )-26

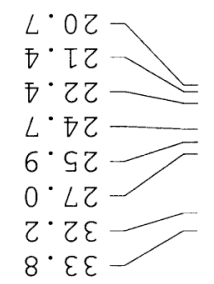

$s \cdot 67-$

$9 \cdot 99$

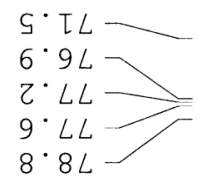

ఐ・帀てて

$6 \cdot 92 \tau$

$6 \cdot \mathrm{LZT}=$

L.8ZT -

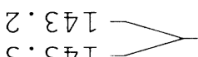

$\varepsilon \cdot \varepsilon \nabla T$

ワ・ワしてい

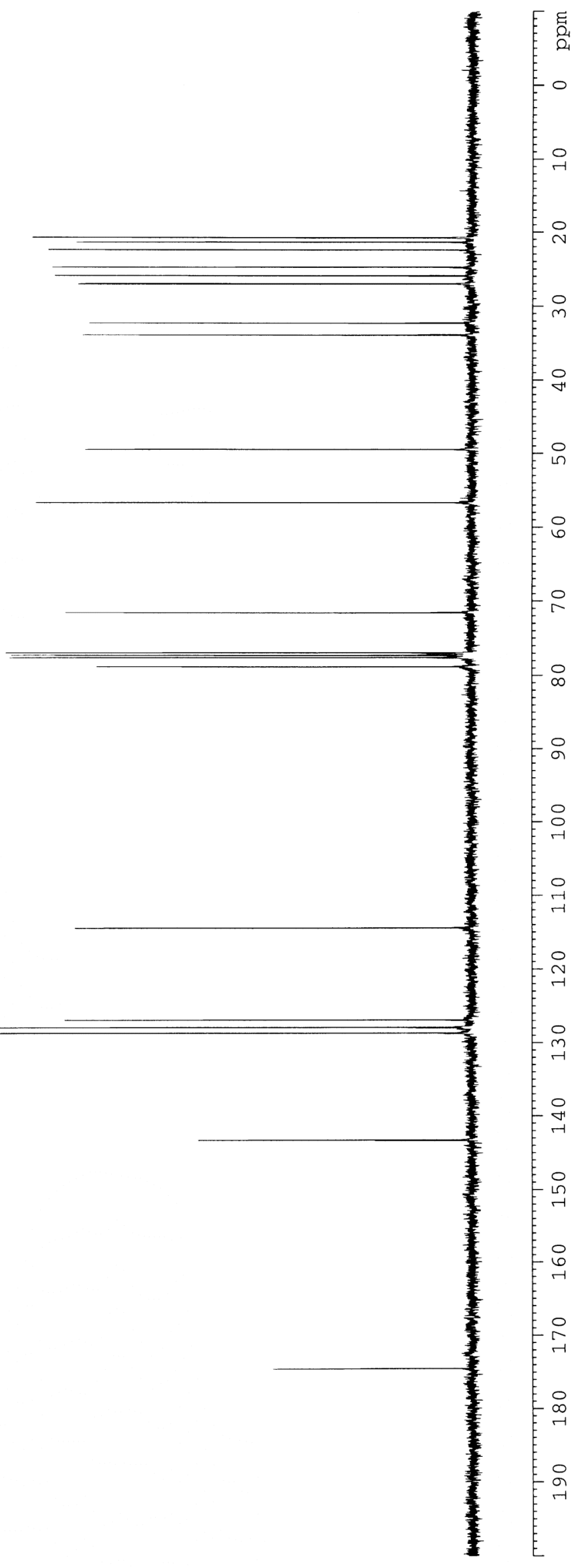


26 (enantiopure) 10:1 mixture of diastereomers

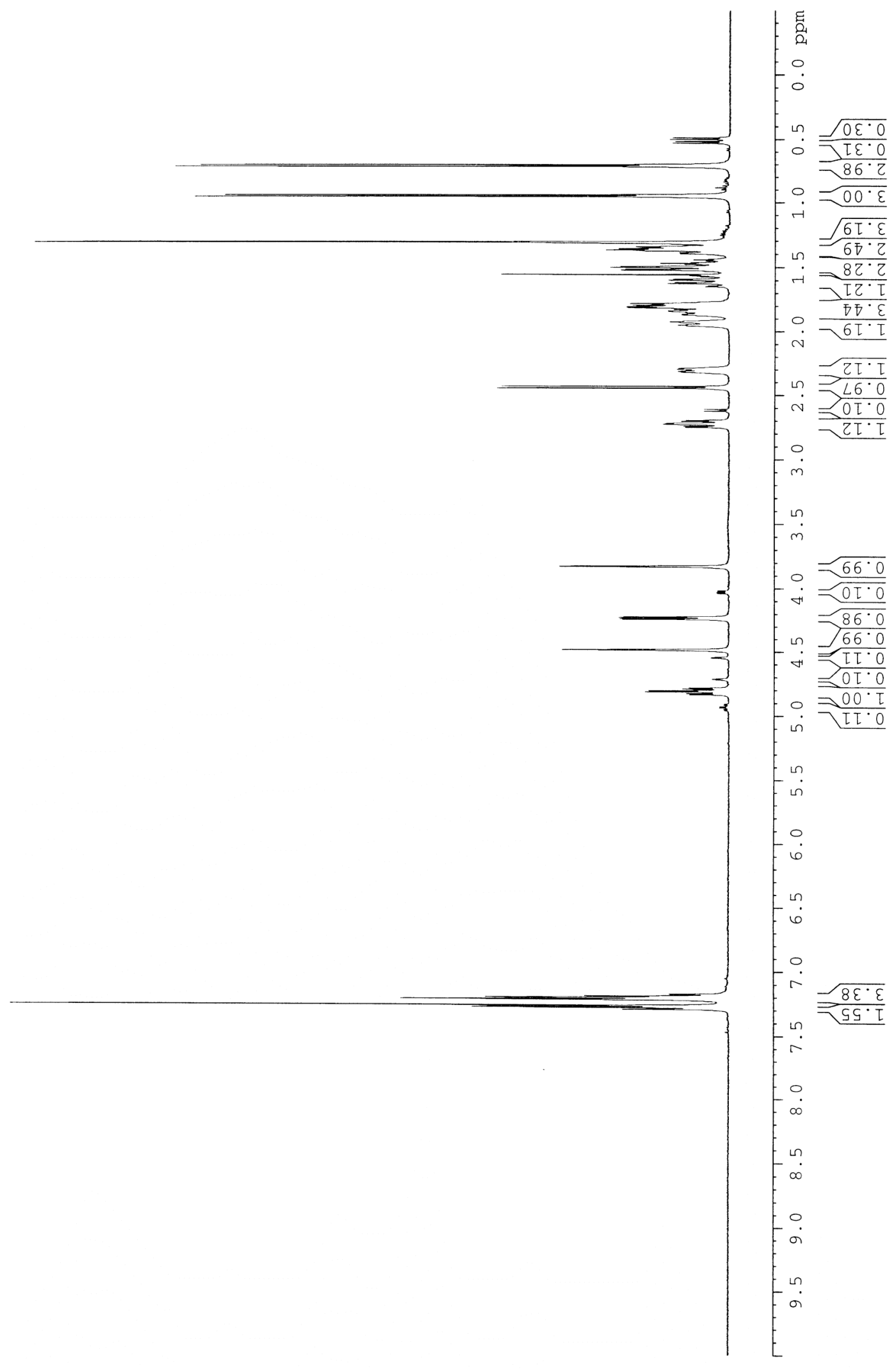




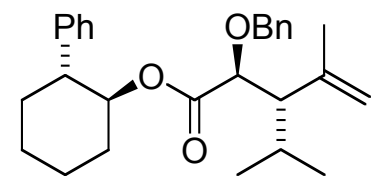

(士)-S1

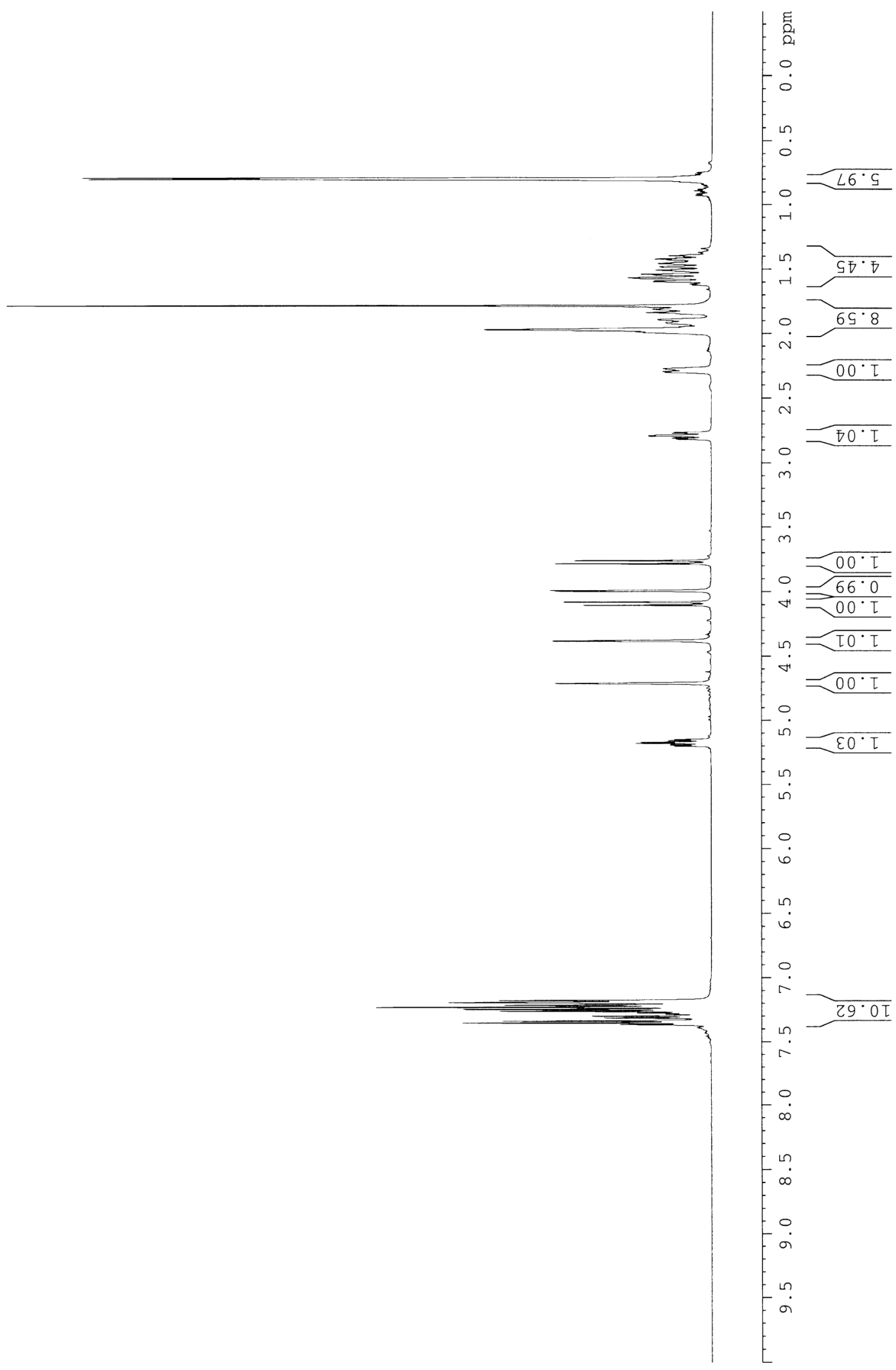


( \pm )-S1

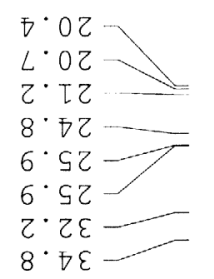

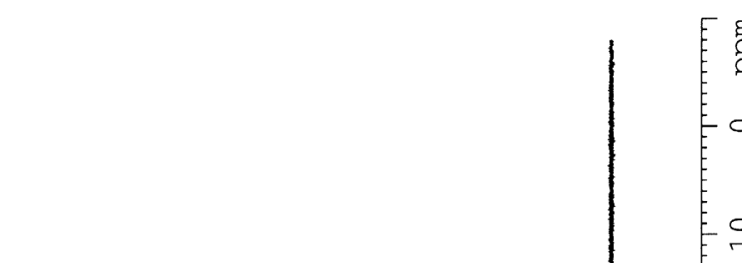

$8 \cdot 60$

$9^{\circ} \angle S$

$0.2 L$

$9^{\circ} \cdot 9 L$
$0 \cdot L L$

$\mathrm{r} \cdot \mathrm{LL} \longrightarrow$

G. $L L$

L. $6 L$
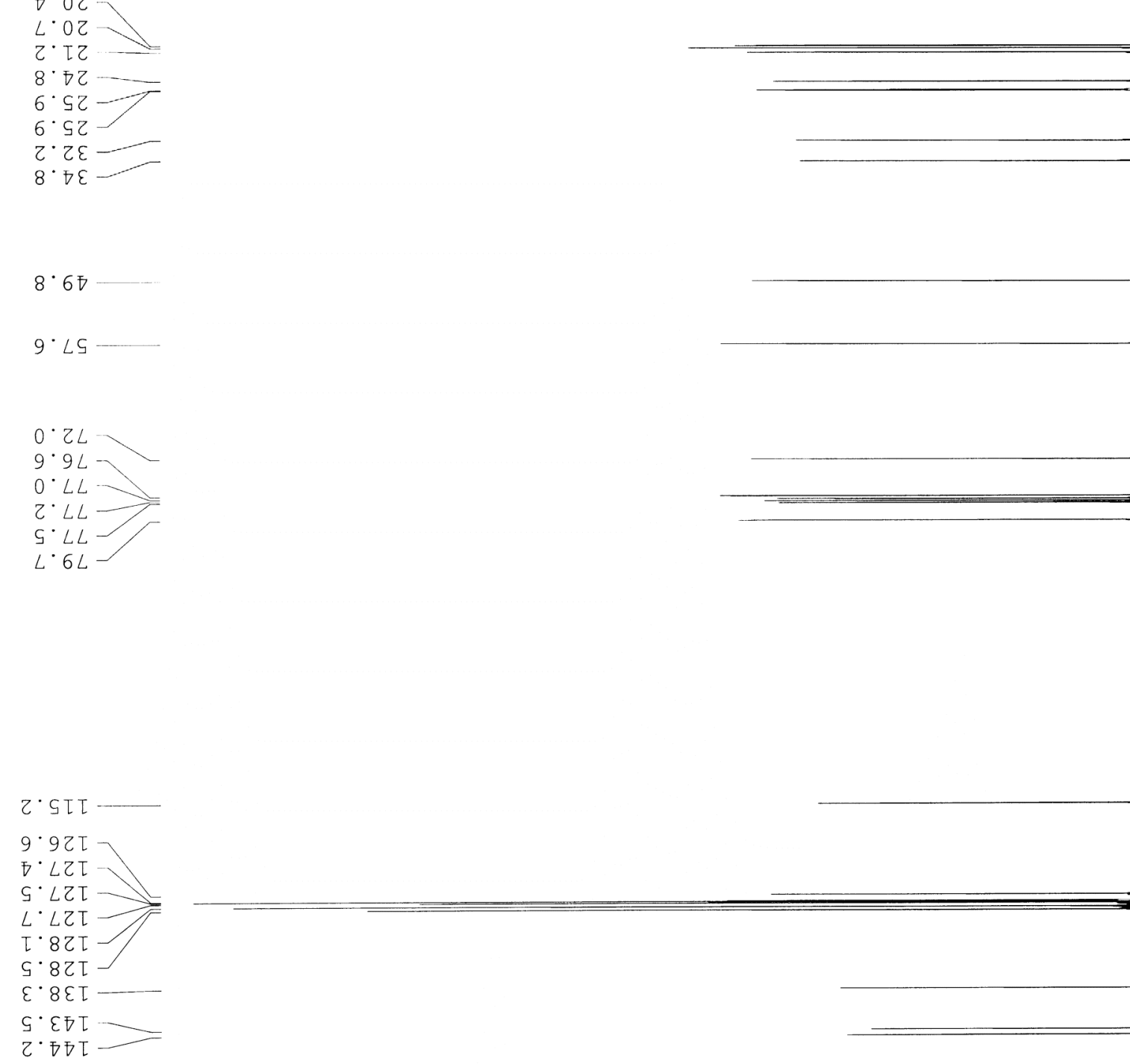

F

우

E음

웅

은

$=0$

E웅

$=$

- 웅

E응

아강

E으묵

각

$=$ 운

ᄀ

$\stackrel{ }{m}$

암

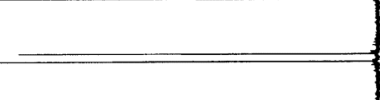

索

옥

=

8

옫

$\varepsilon \cdot 2 L \tau$

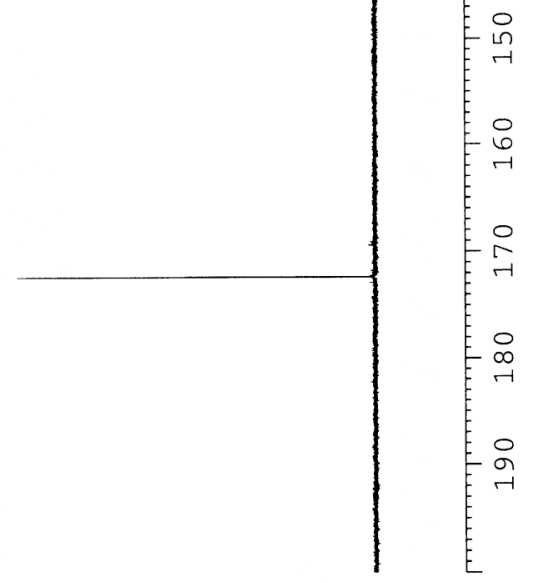




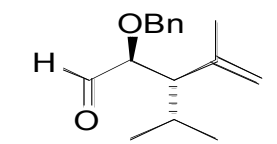

( \pm )-27

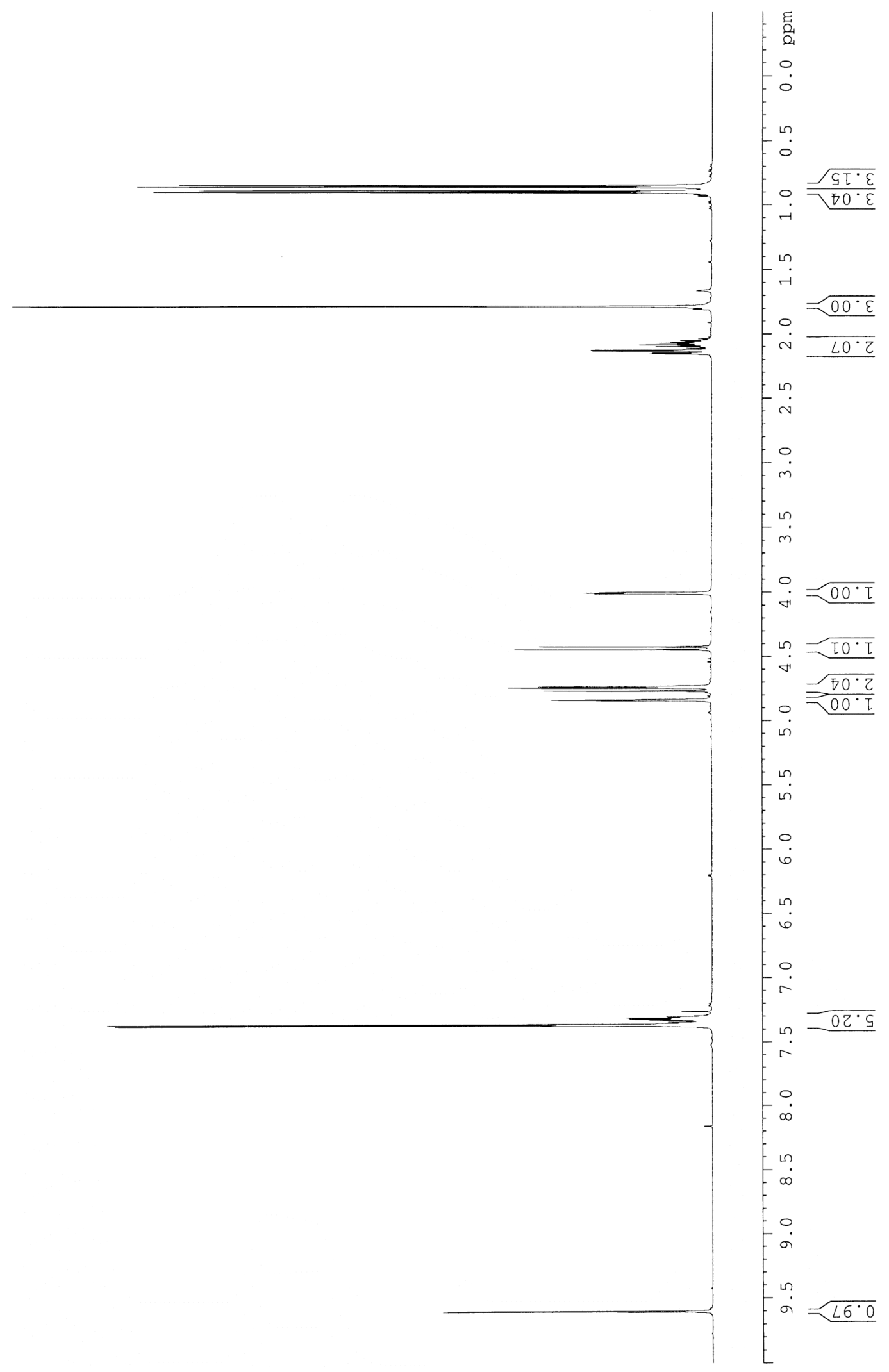


( \pm )-27

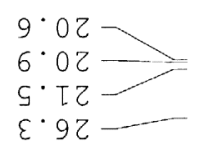

$\varepsilon \cdot 9 \varsigma$

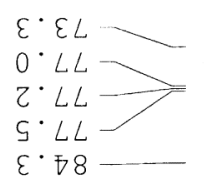

$9 \cdot \operatorname{stL}$

$6^{\circ}$ LZT

$0.8 \mathrm{ZT}$

$9 \cdot 82 \tau$

8. LET

O. 五

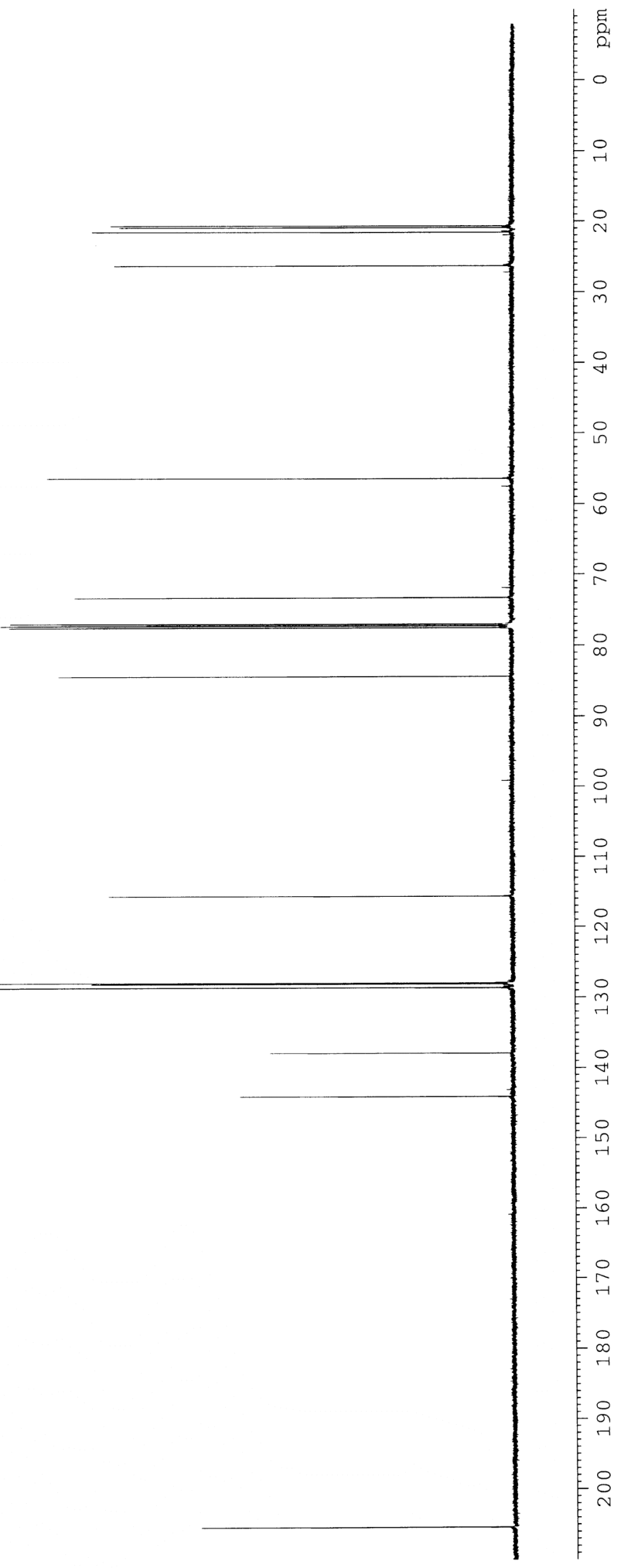




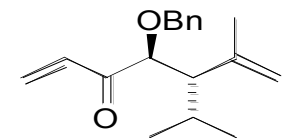

(士)-28

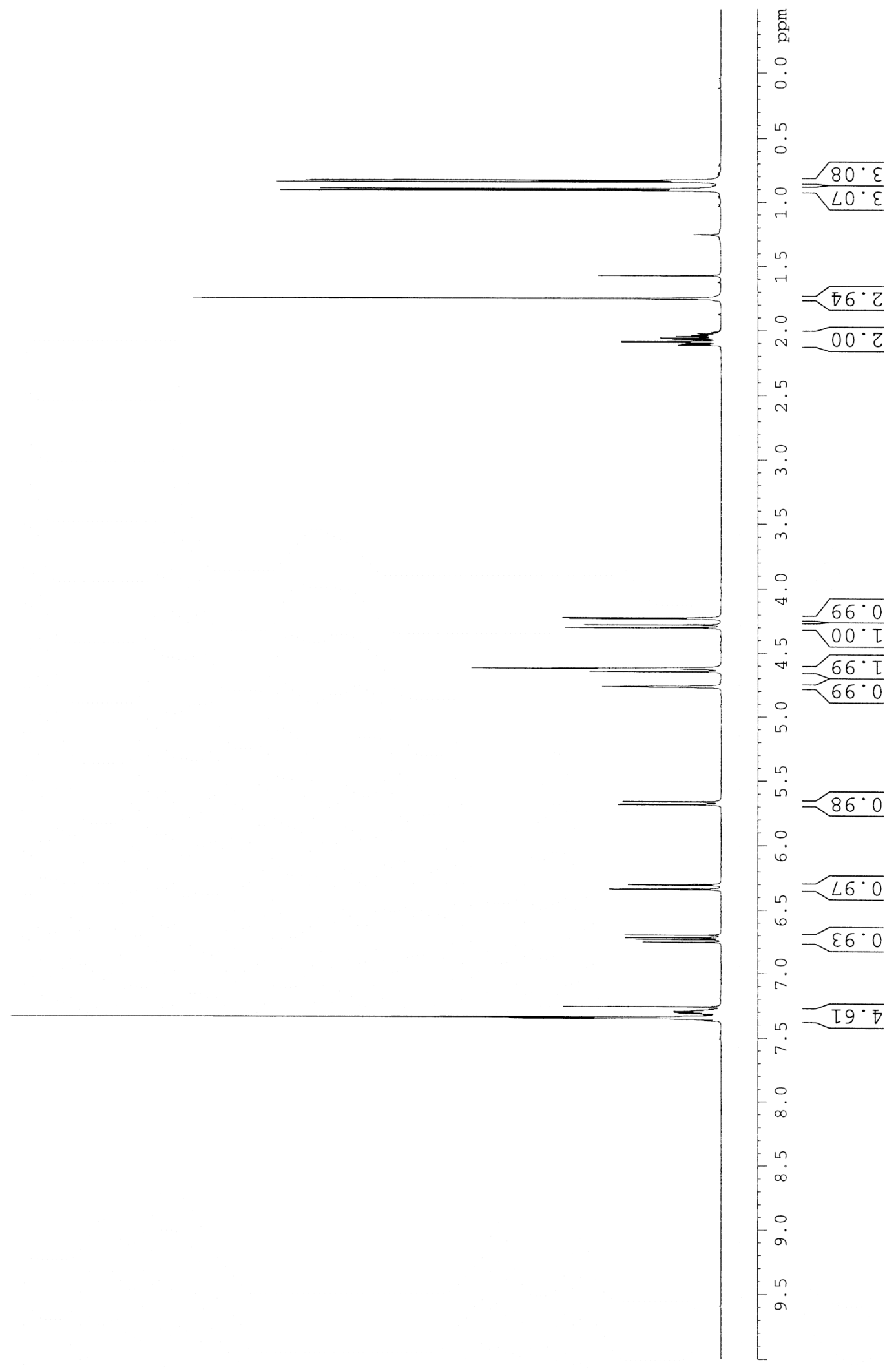


( \pm )-28
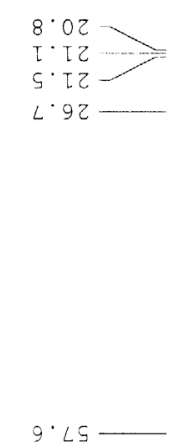

y $9 t$
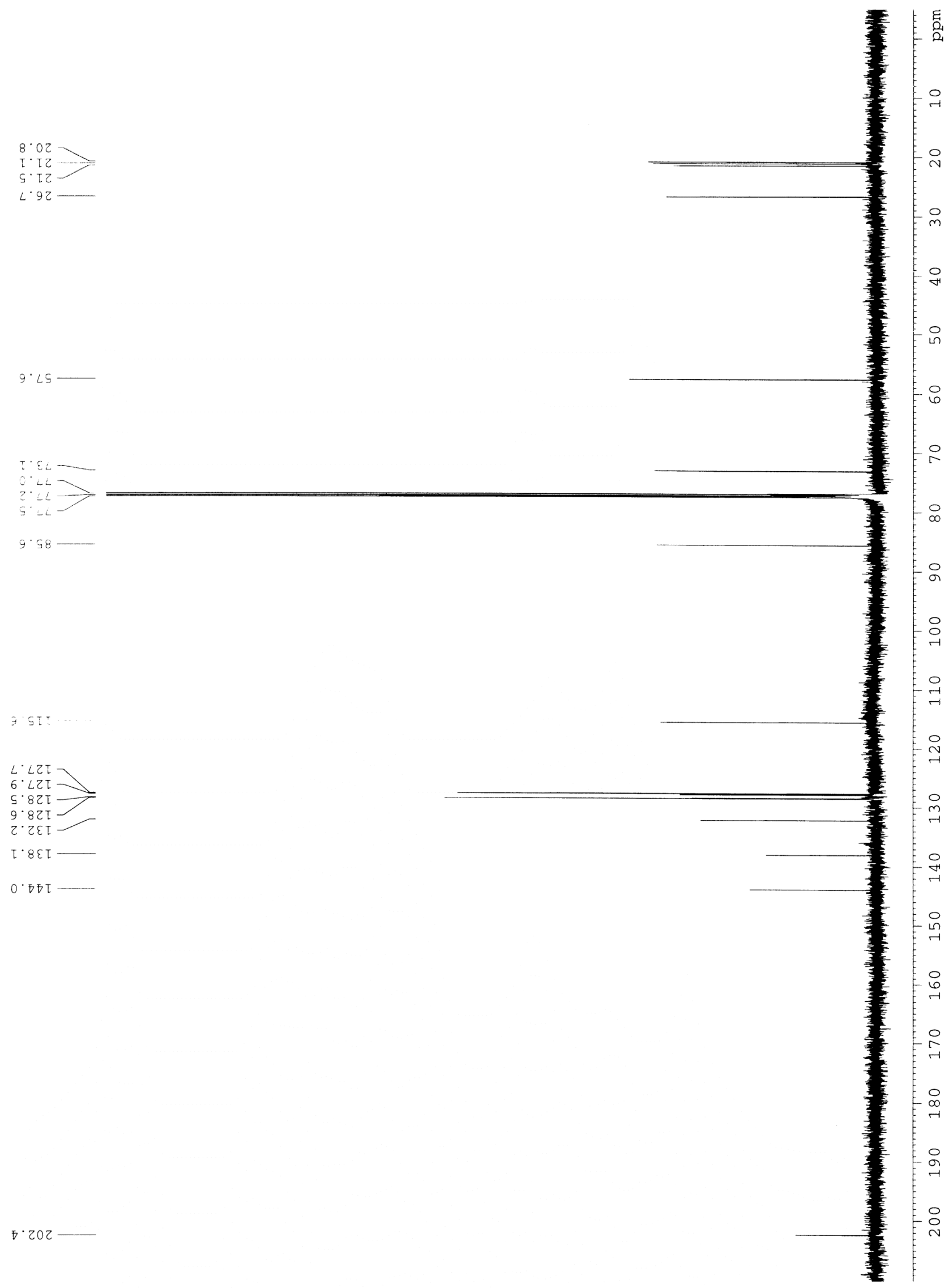


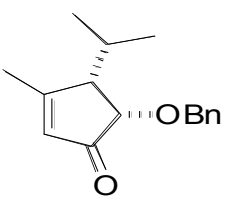

(-)-29

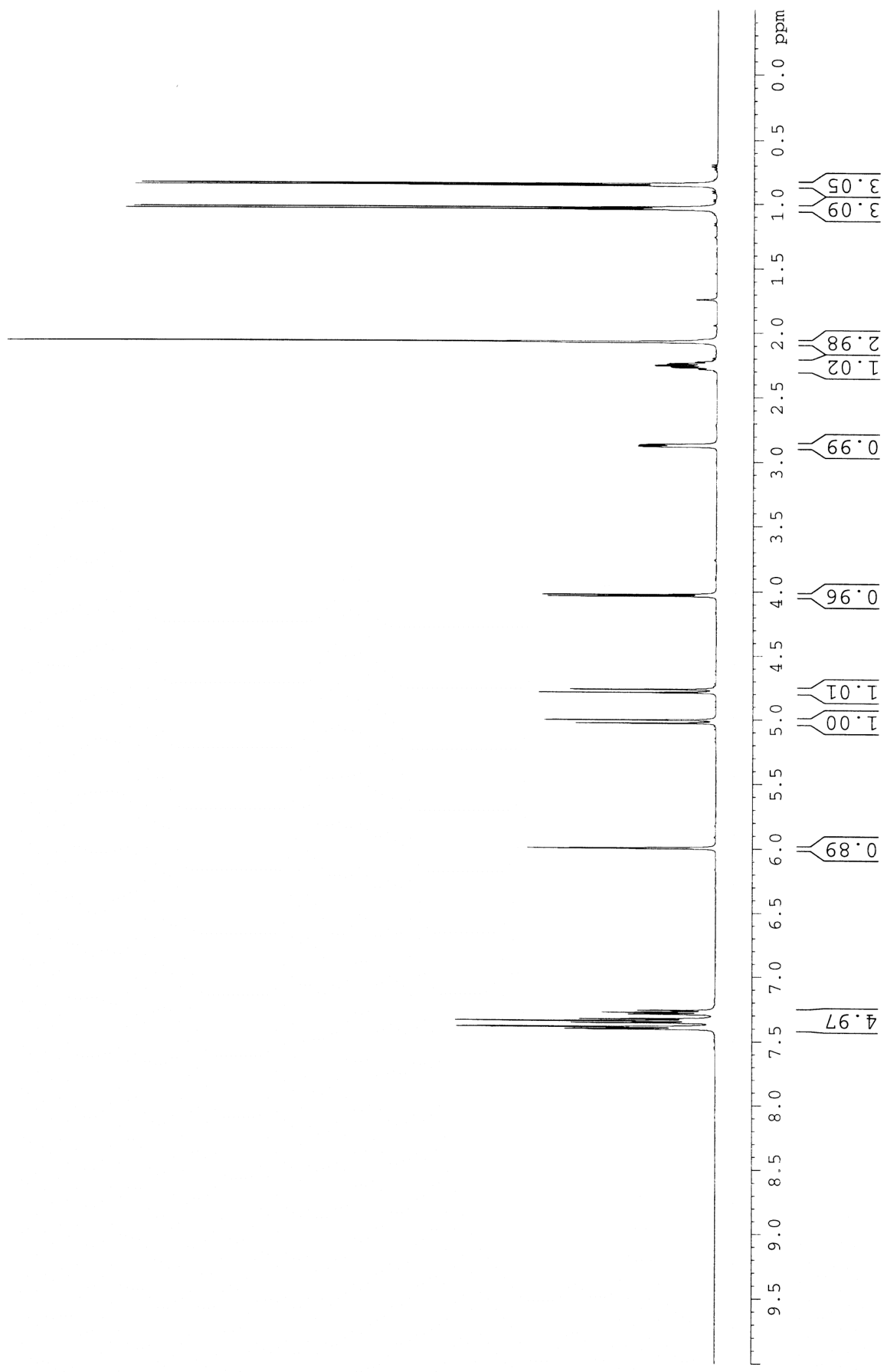


(-)-29

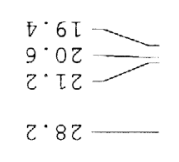

$6 \cdot \varepsilon 5$

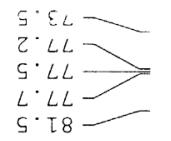

$0.82 \mathrm{O}$
$0.82 \mathrm{Z}$
$8.82 \mathrm{Z}$
$8.62 \mathrm{~T}$

$9 \cdot 8 \varepsilon I-$

$9 \cdot S L T-$

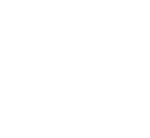

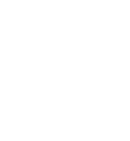
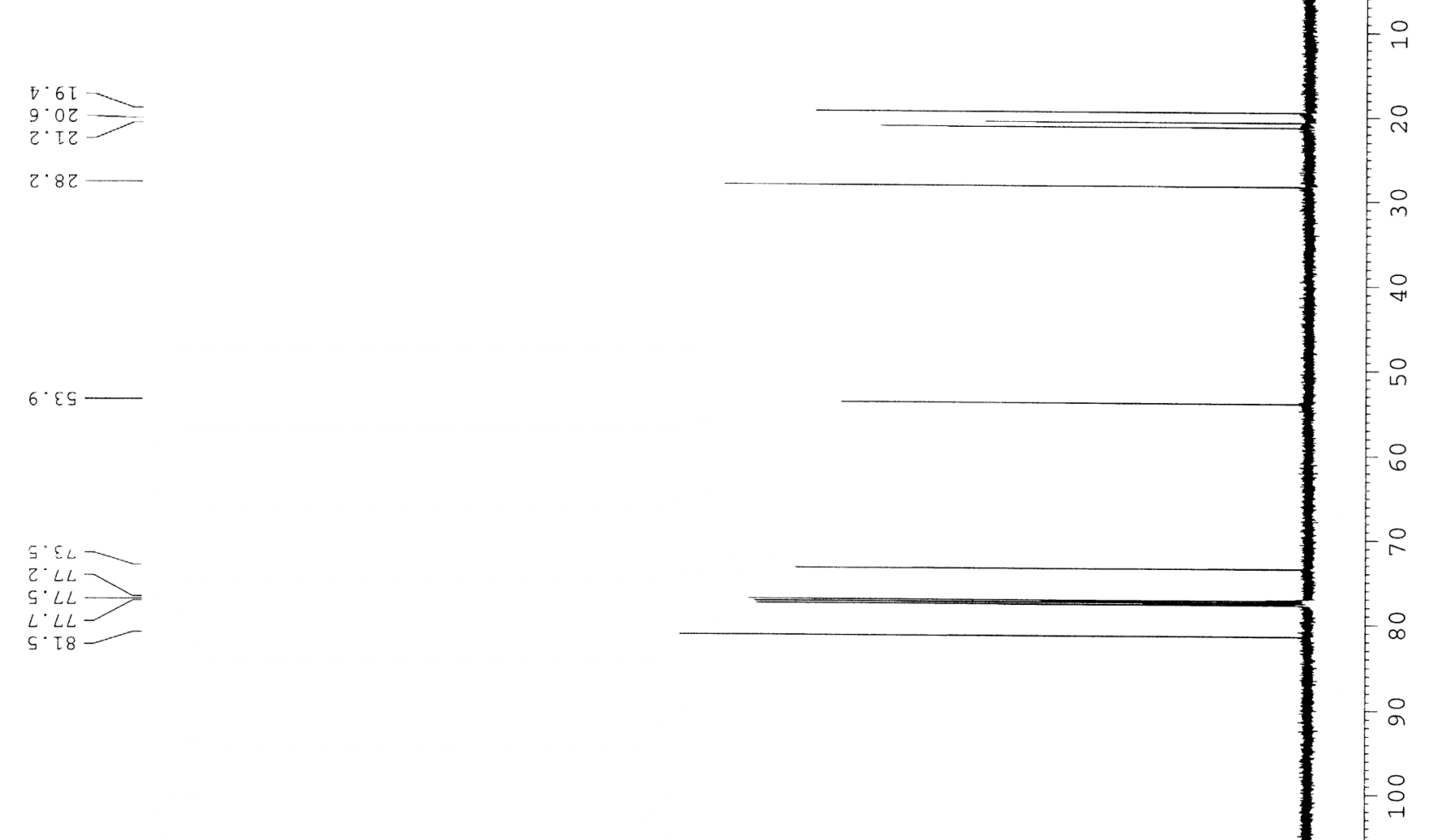

$9 \cdot \mathrm{LOZ}$

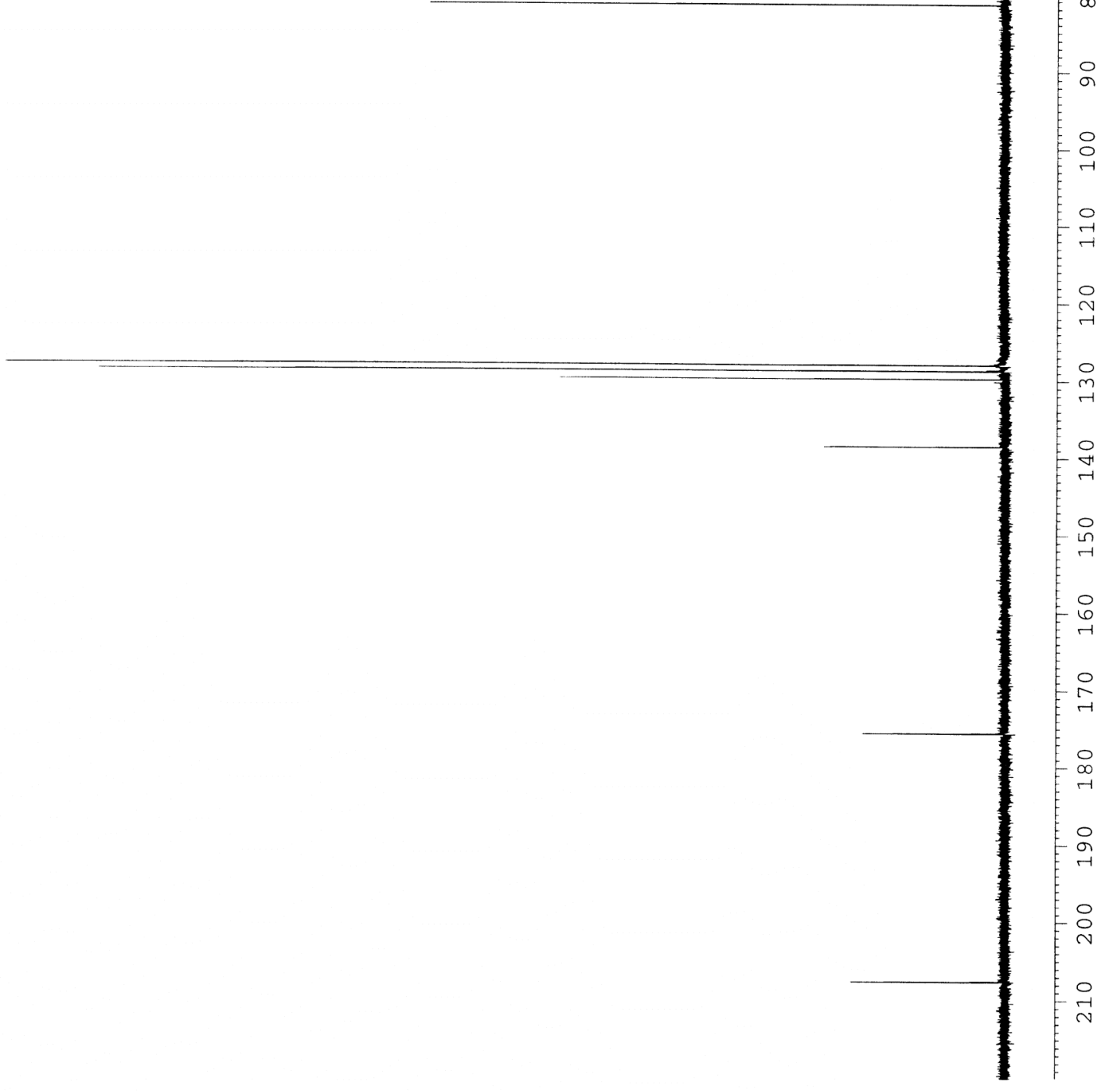



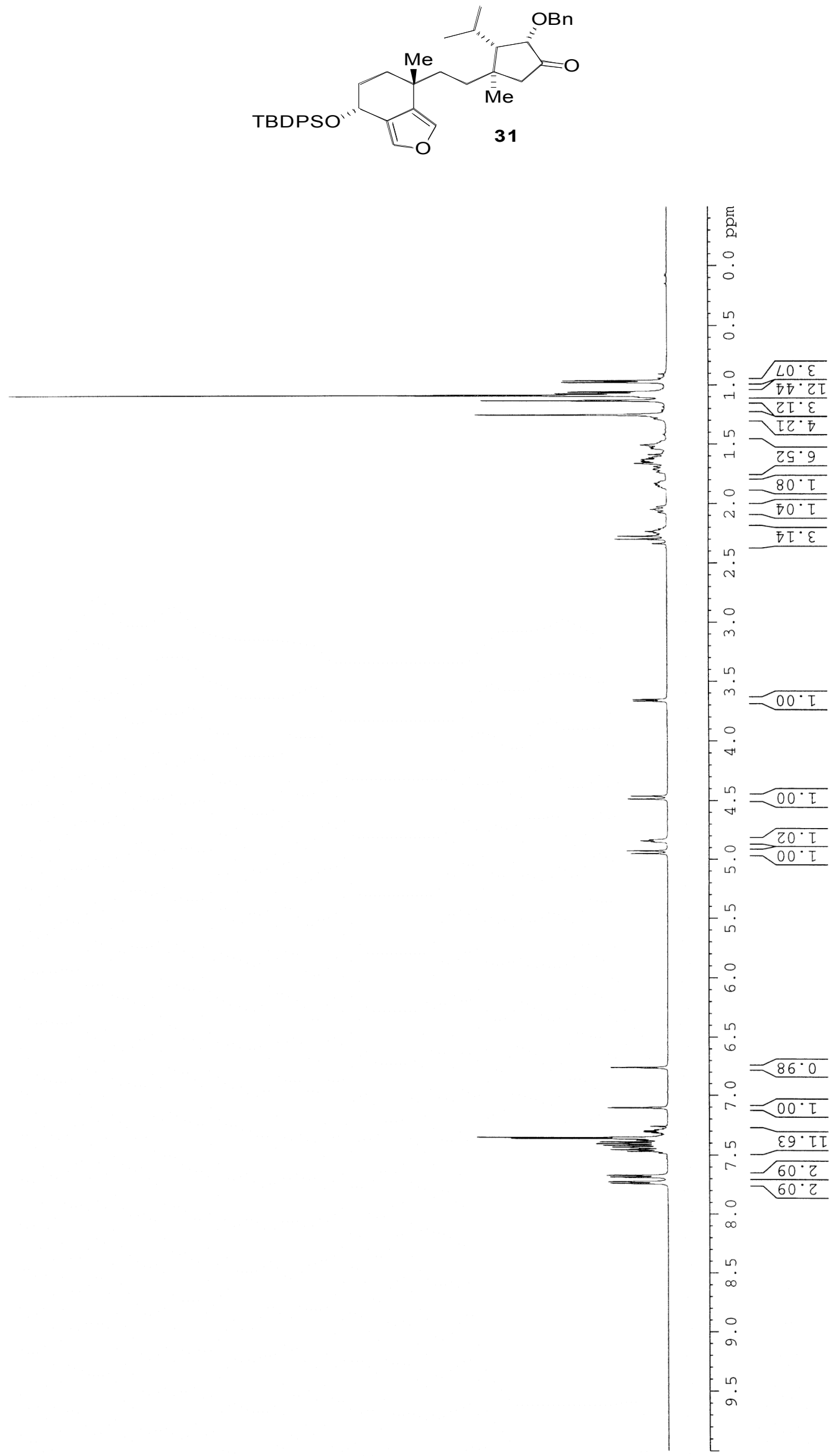


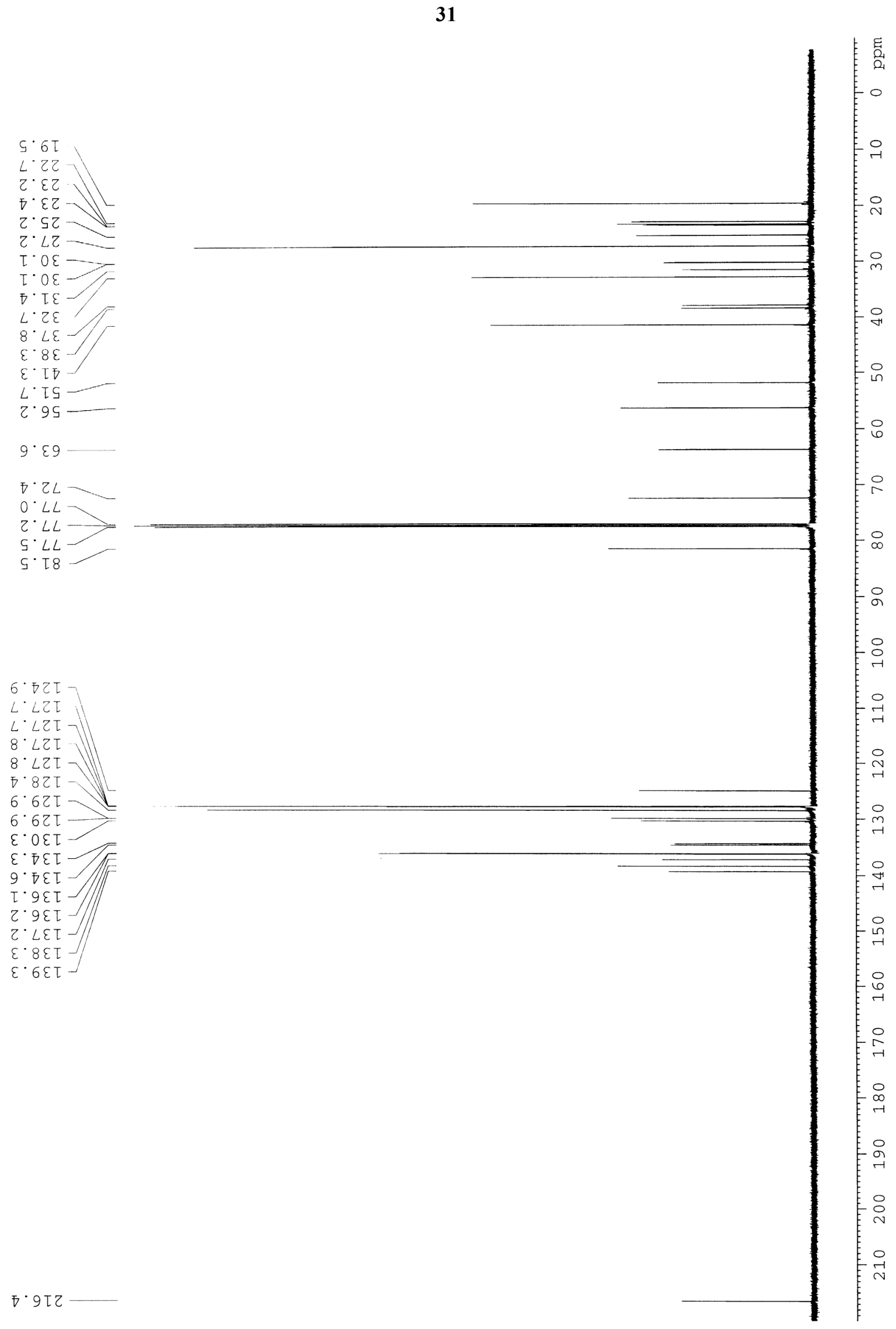



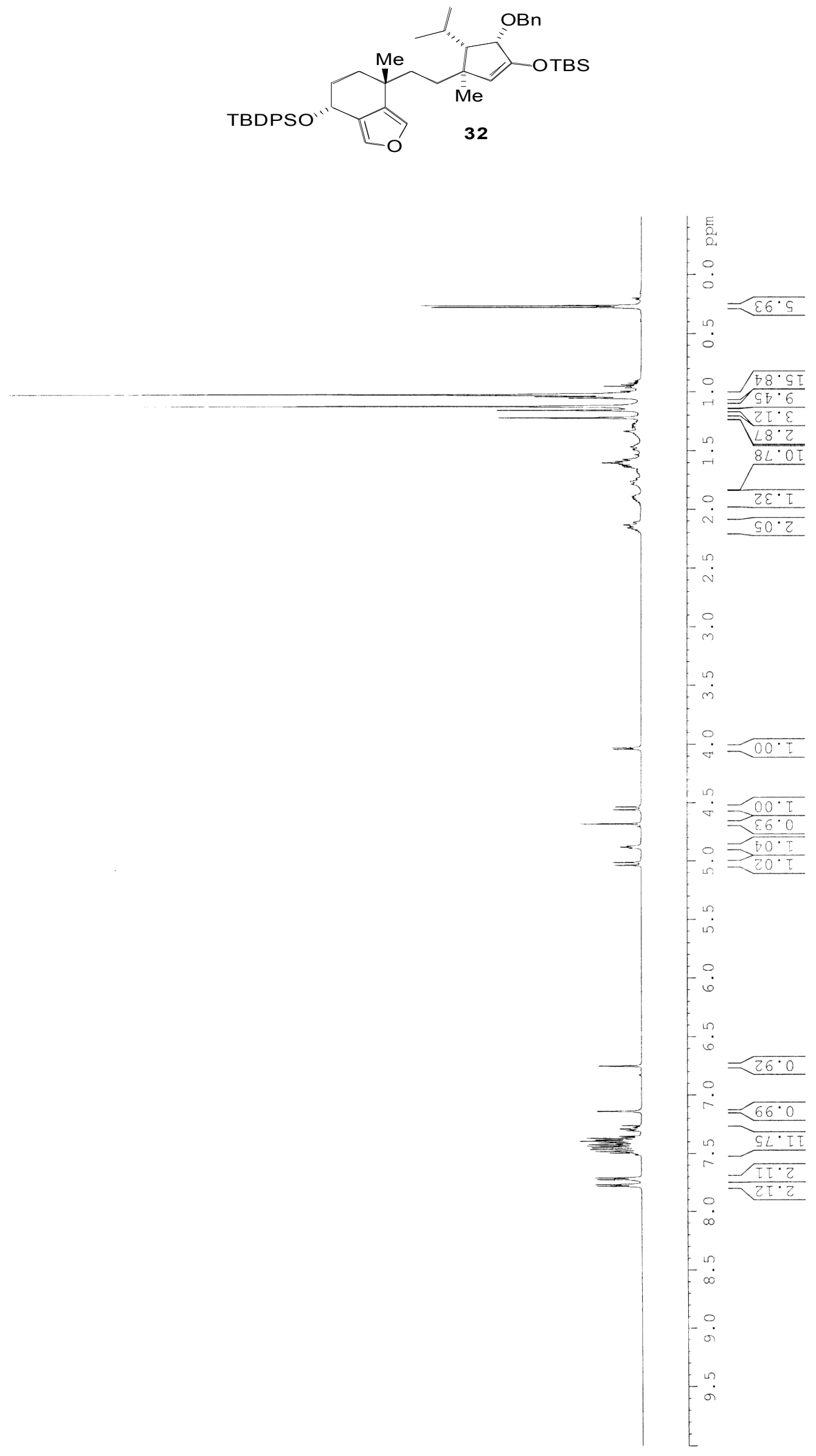

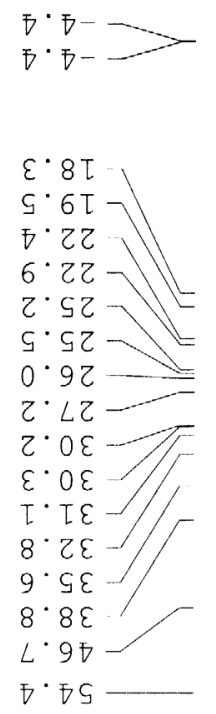

$9 \cdot \varepsilon 9$
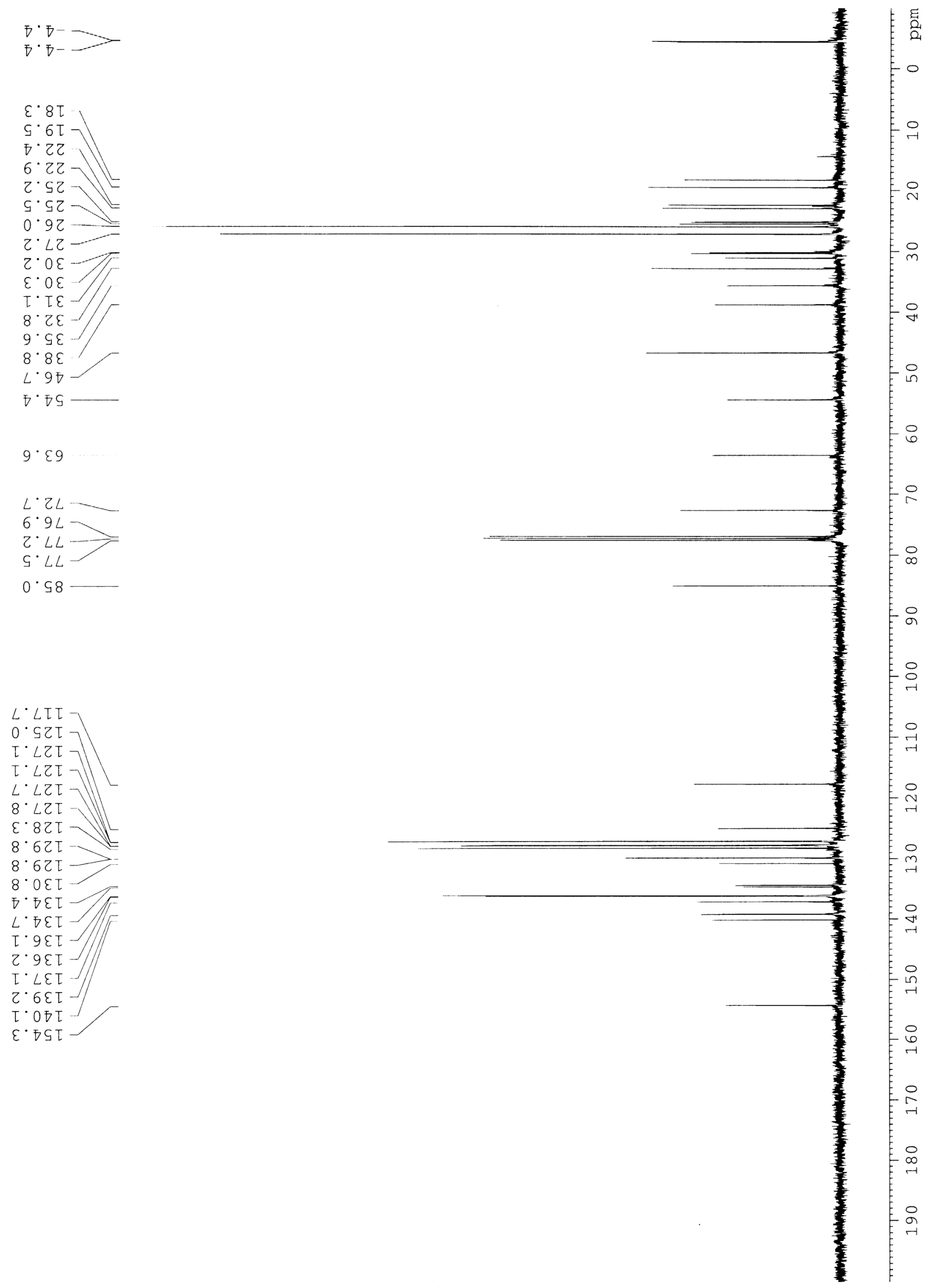


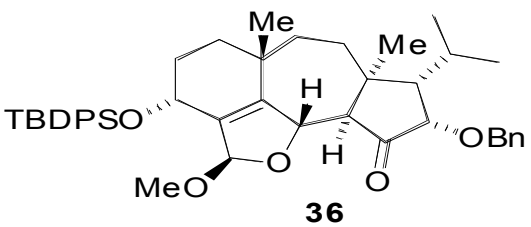

room temperature

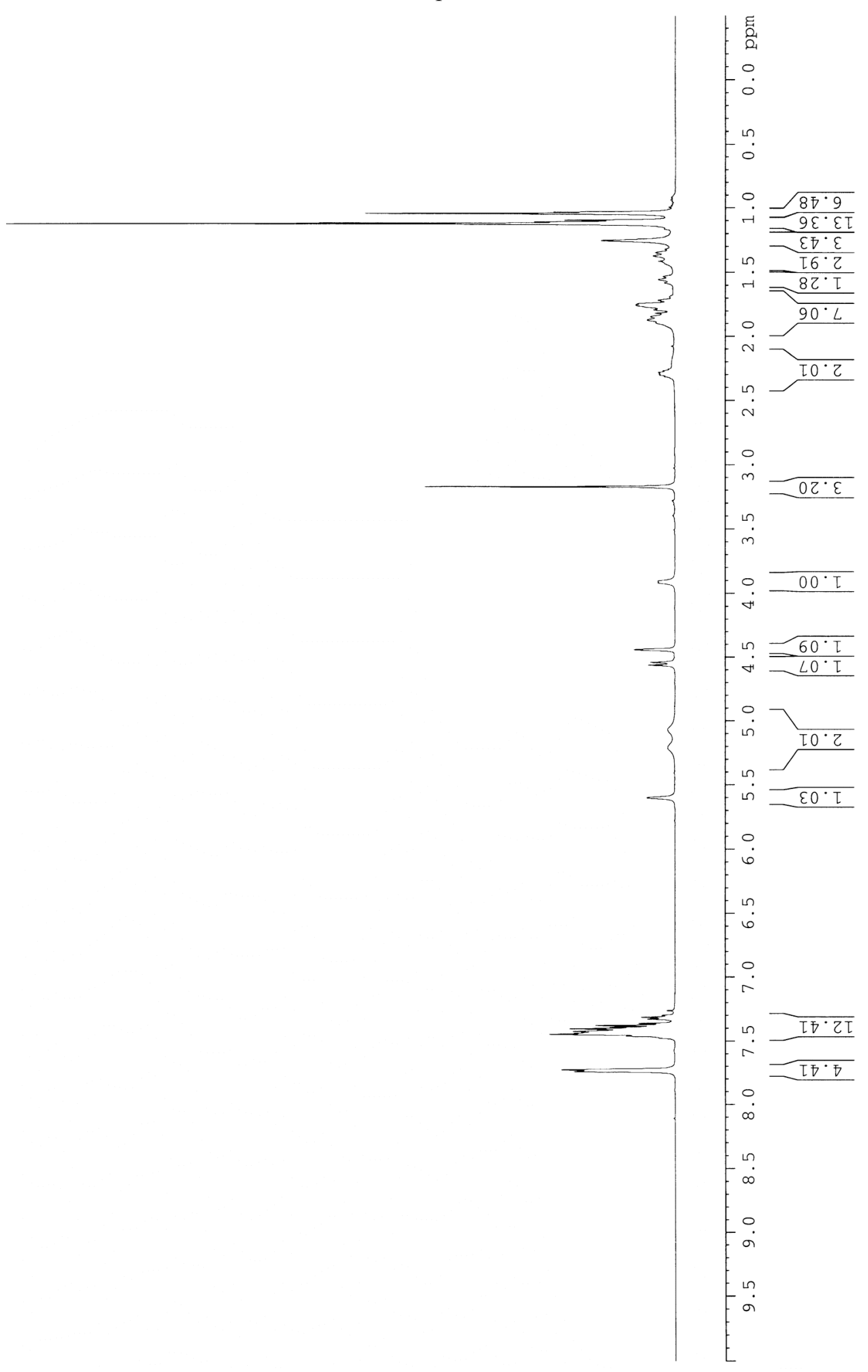


$36\left(53^{\circ} \mathrm{C}\right)$

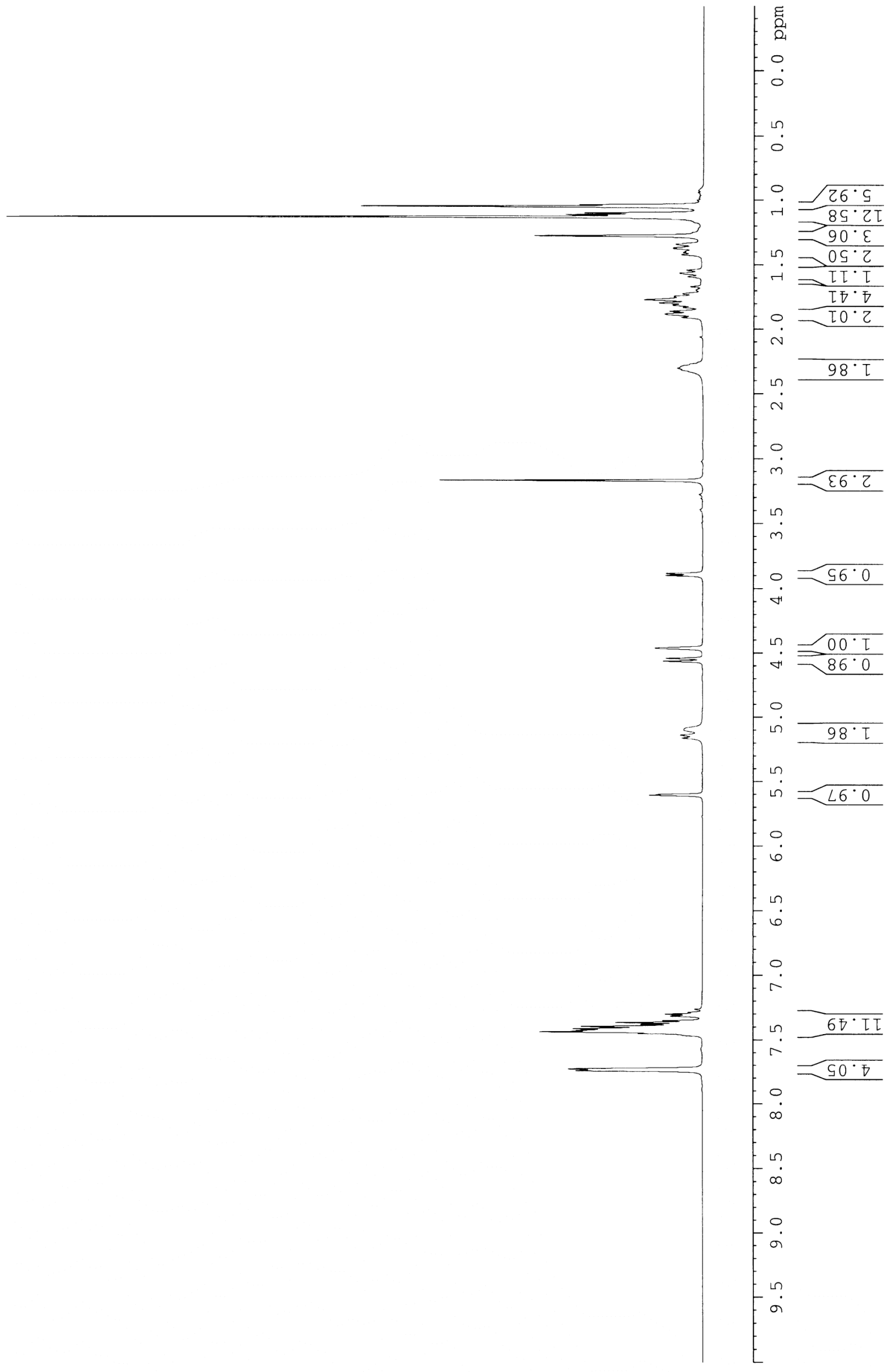




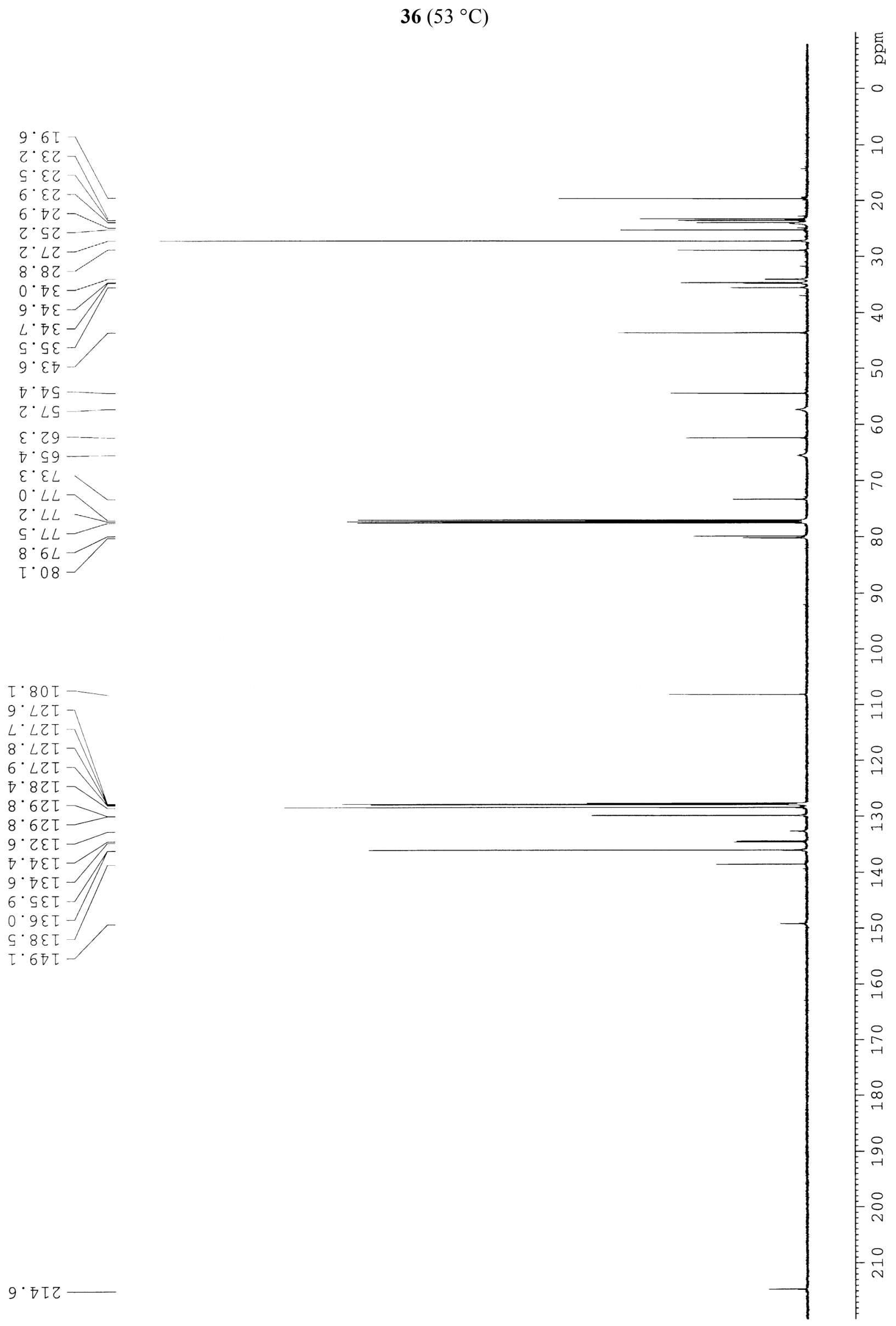



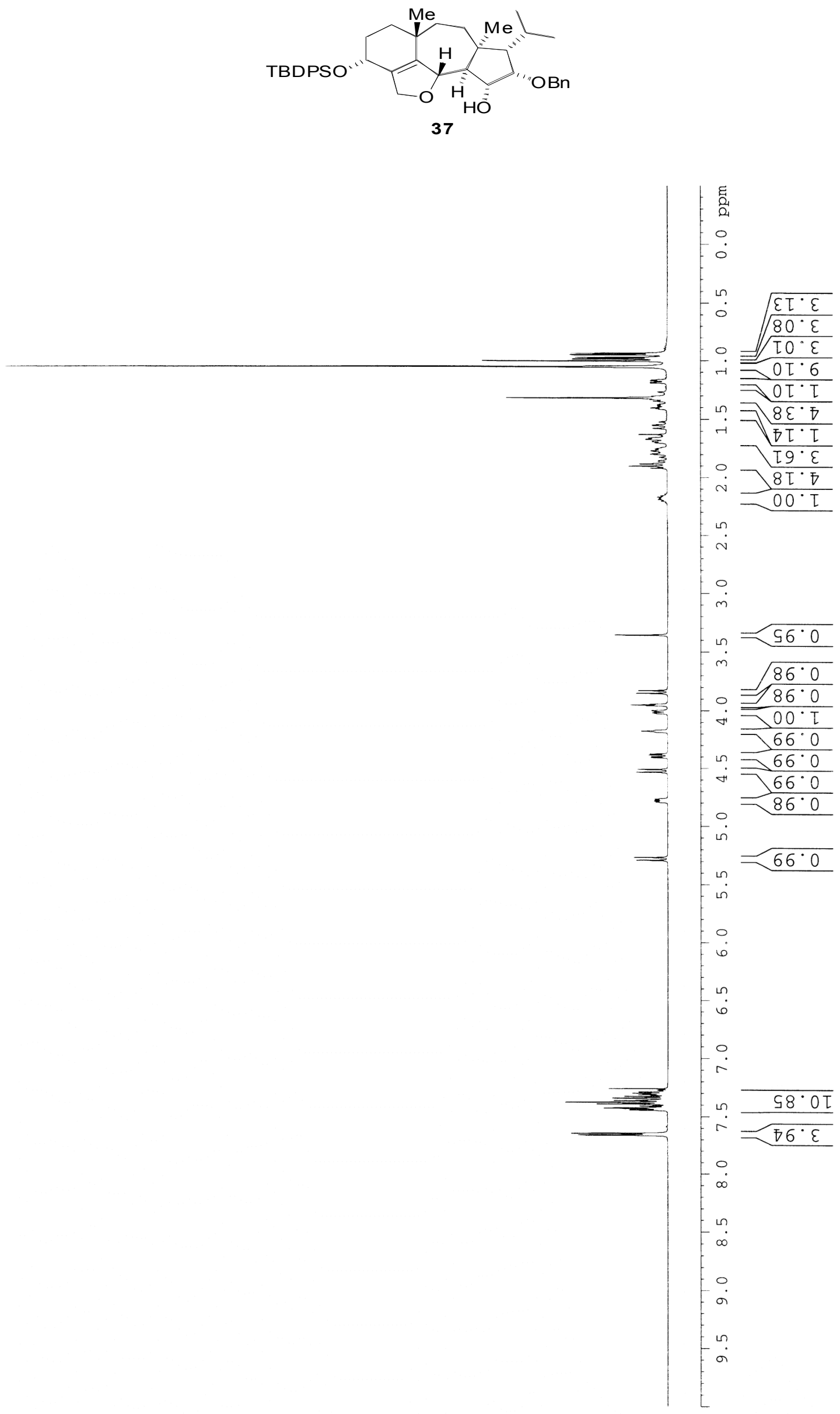


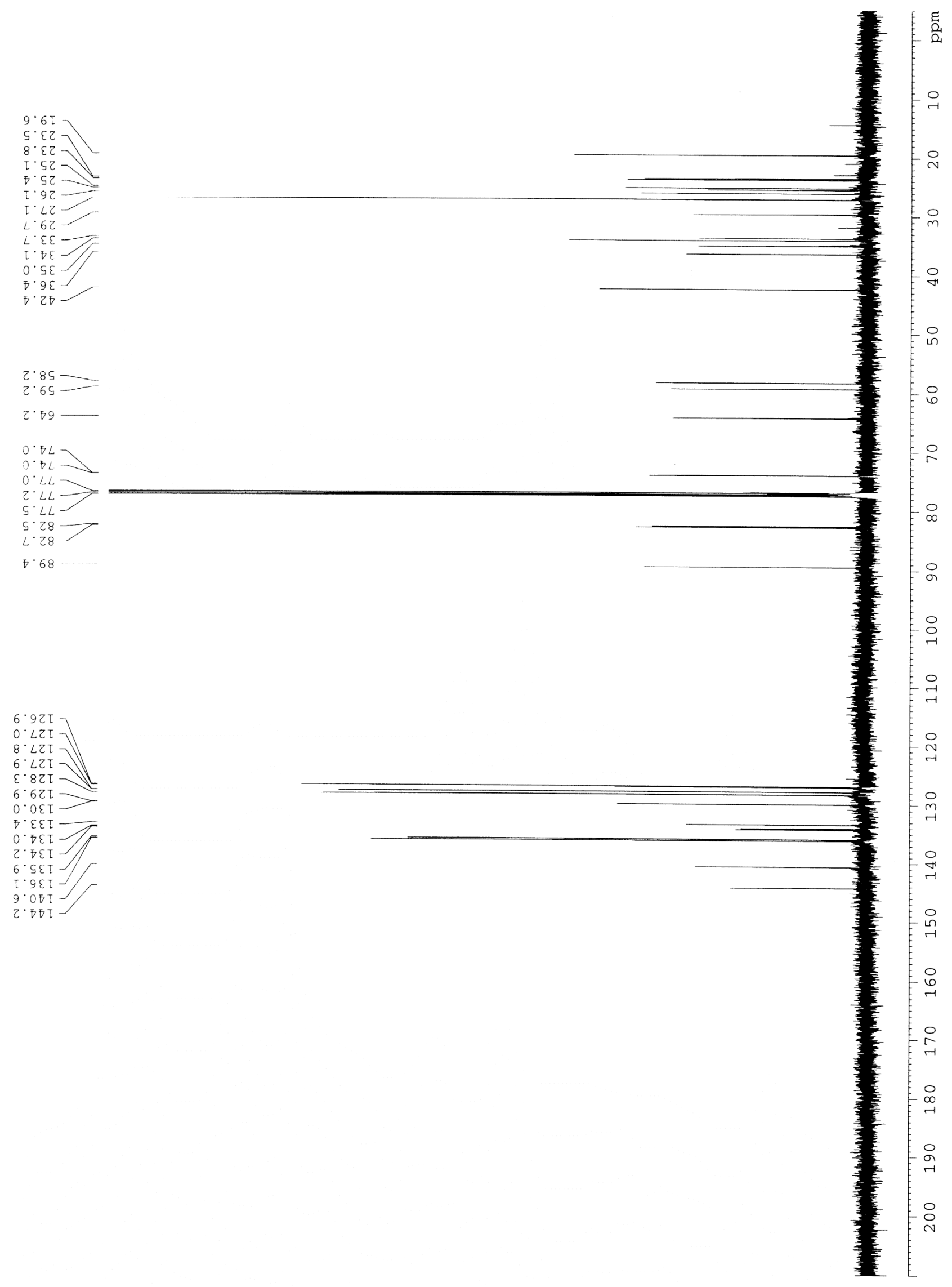


37 (gCOSY)

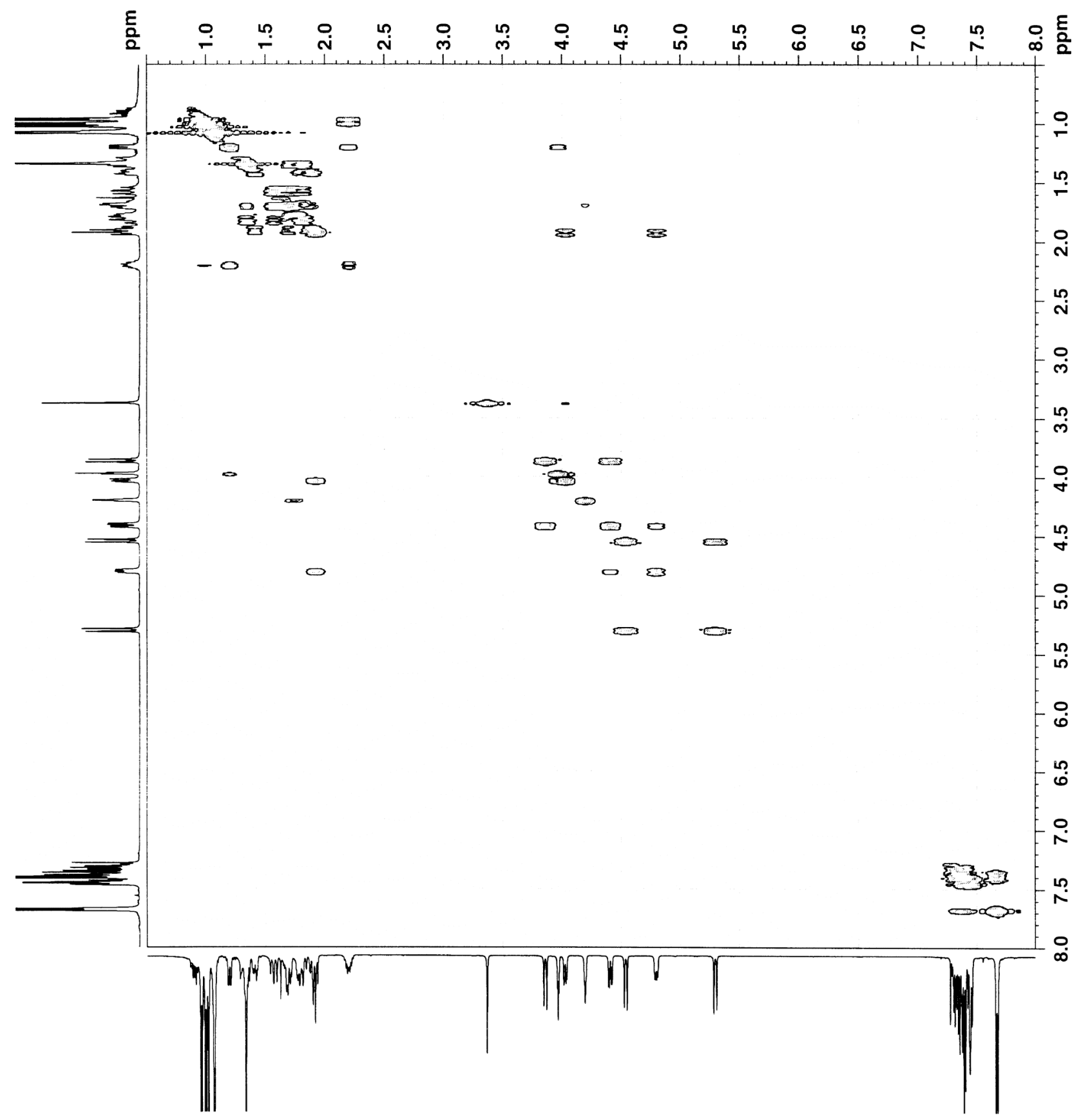


37 (gNOESY)
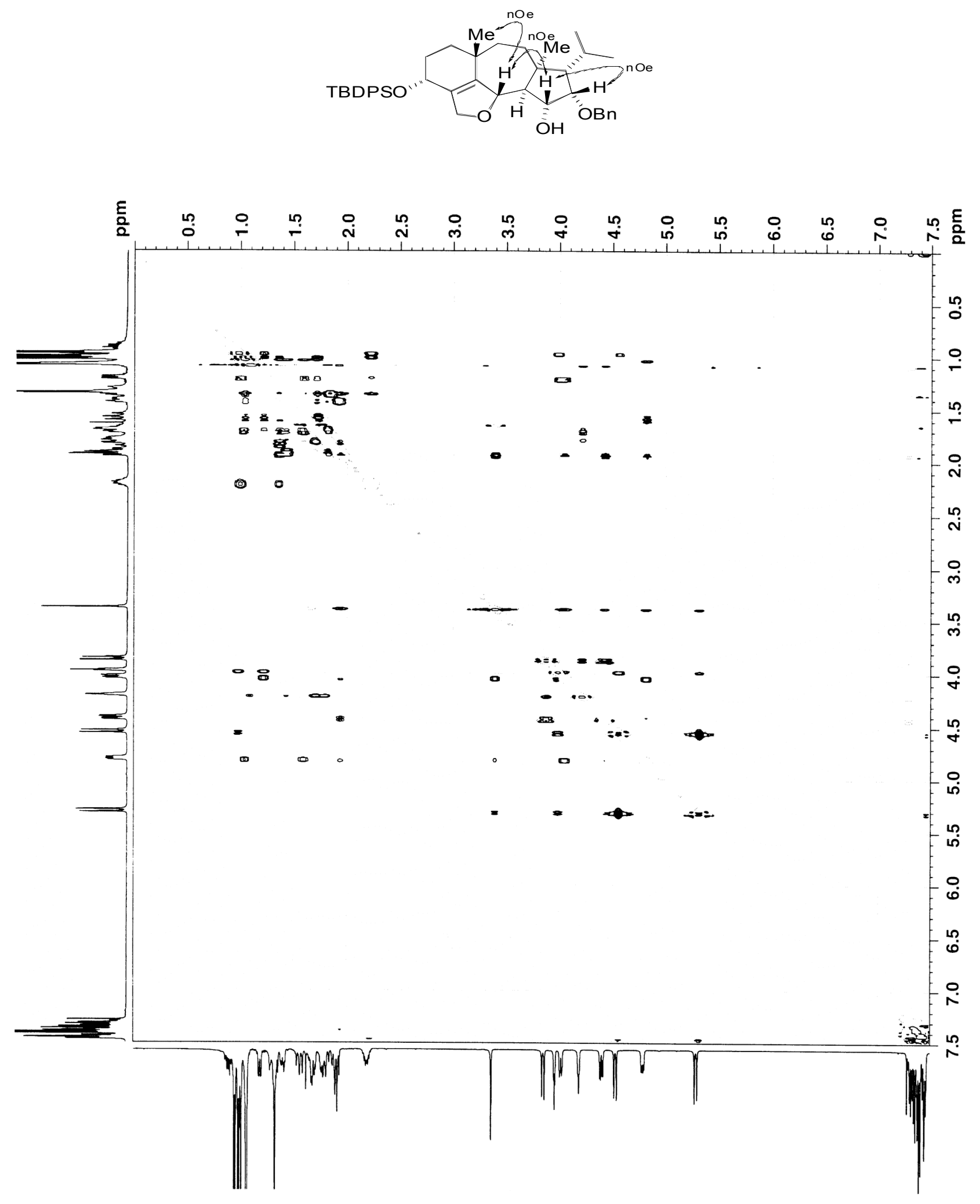


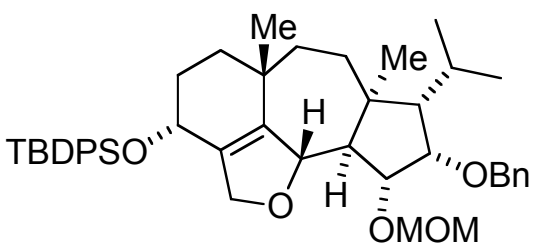

S2

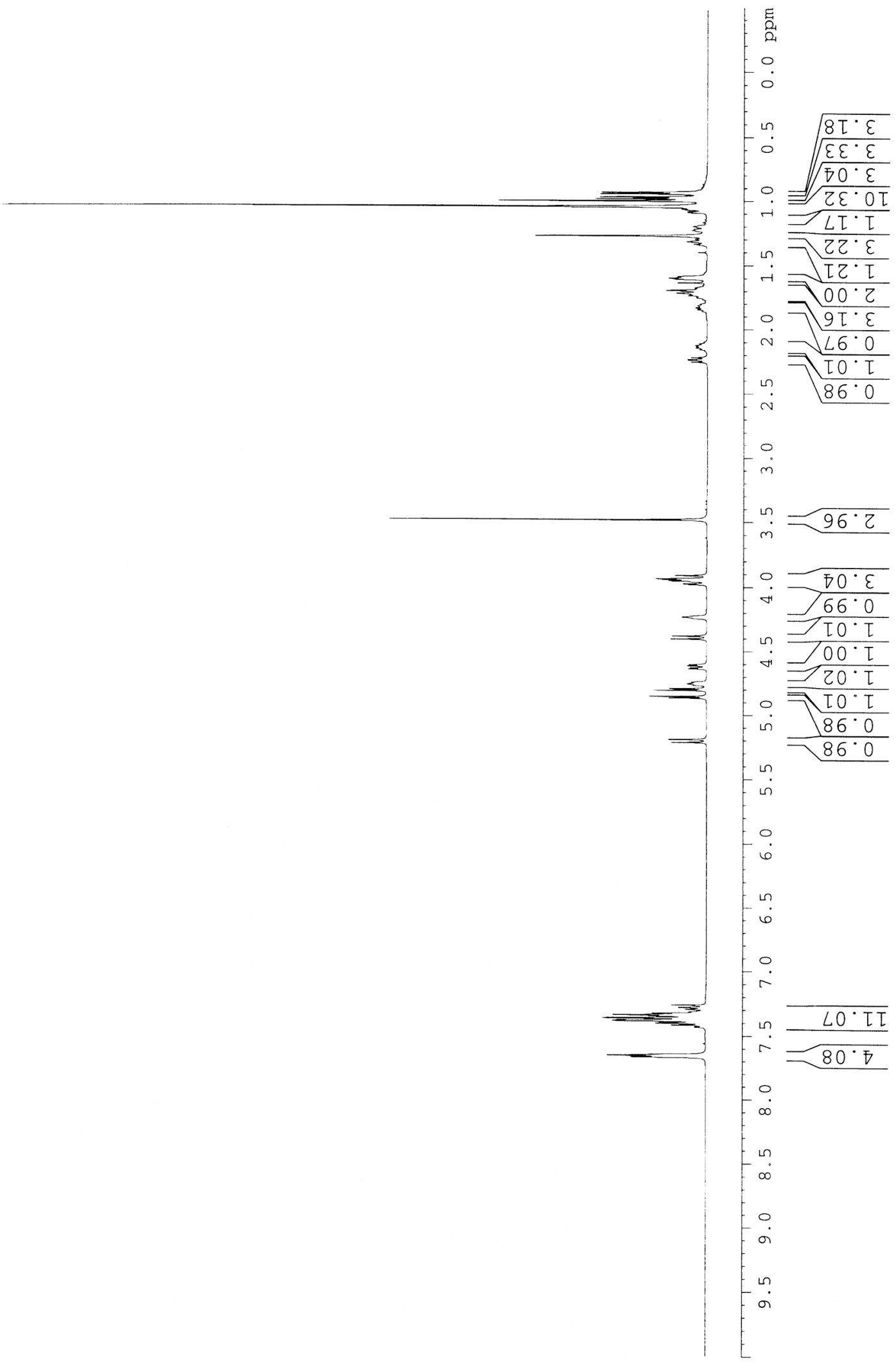


S2

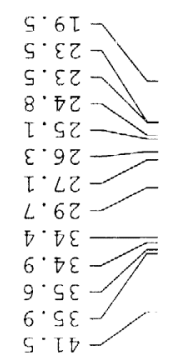

$6.55 \ldots$
4.95

$0.85=$

$L \cdot \subseteq 9$

$L \cdot \varepsilon L \Longrightarrow$
$L \cdot \varepsilon L$

O $L L \longrightarrow$

与.

9.78-

$6 \cdot 56$

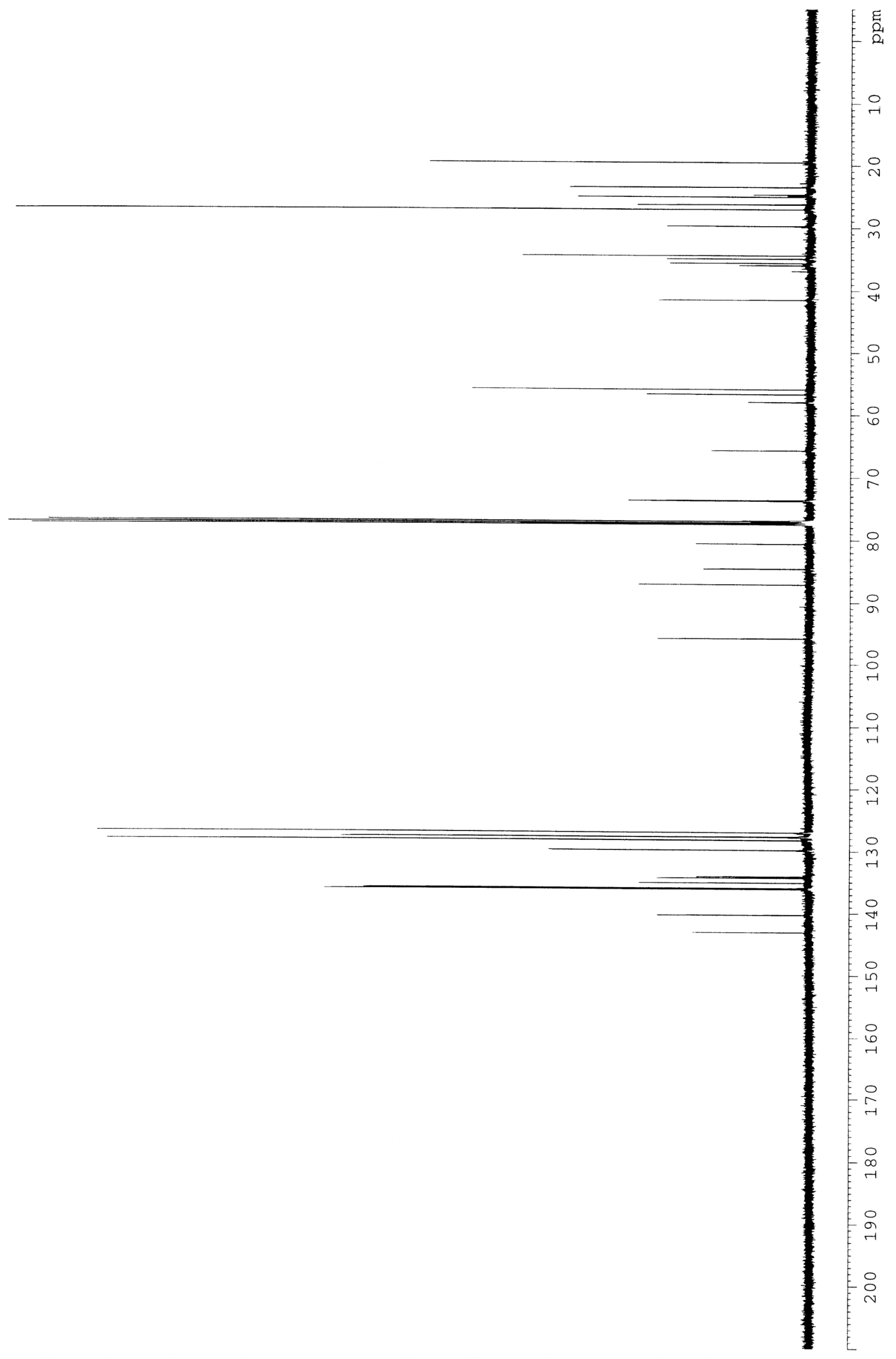




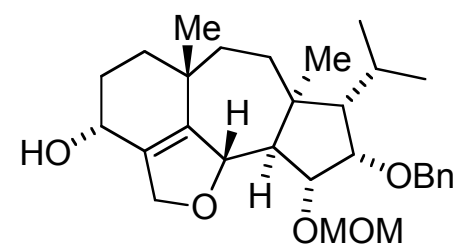

S3

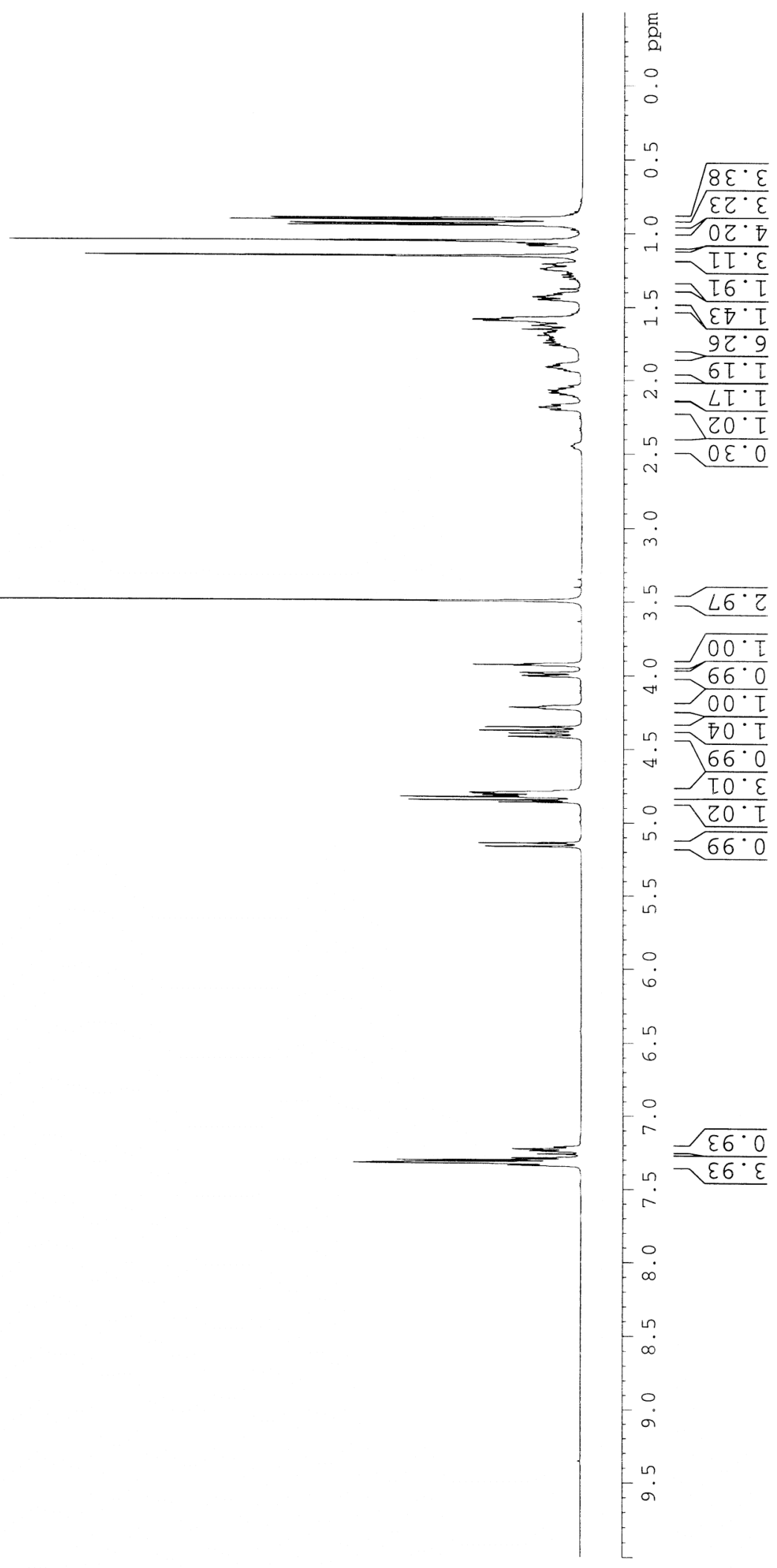



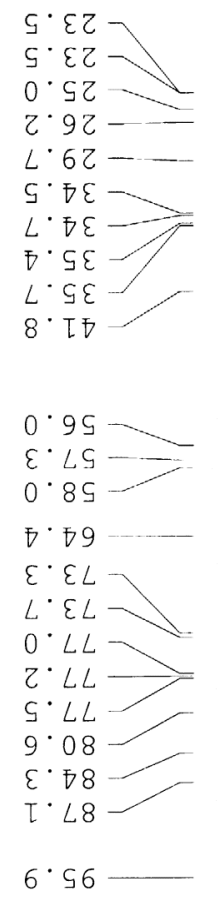

$6.9 Z I$

$0 \cdot \angle Z I$

乙. $82 \tau$

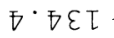

$\tau \cdot 0 \nabla \tau$

I. 五

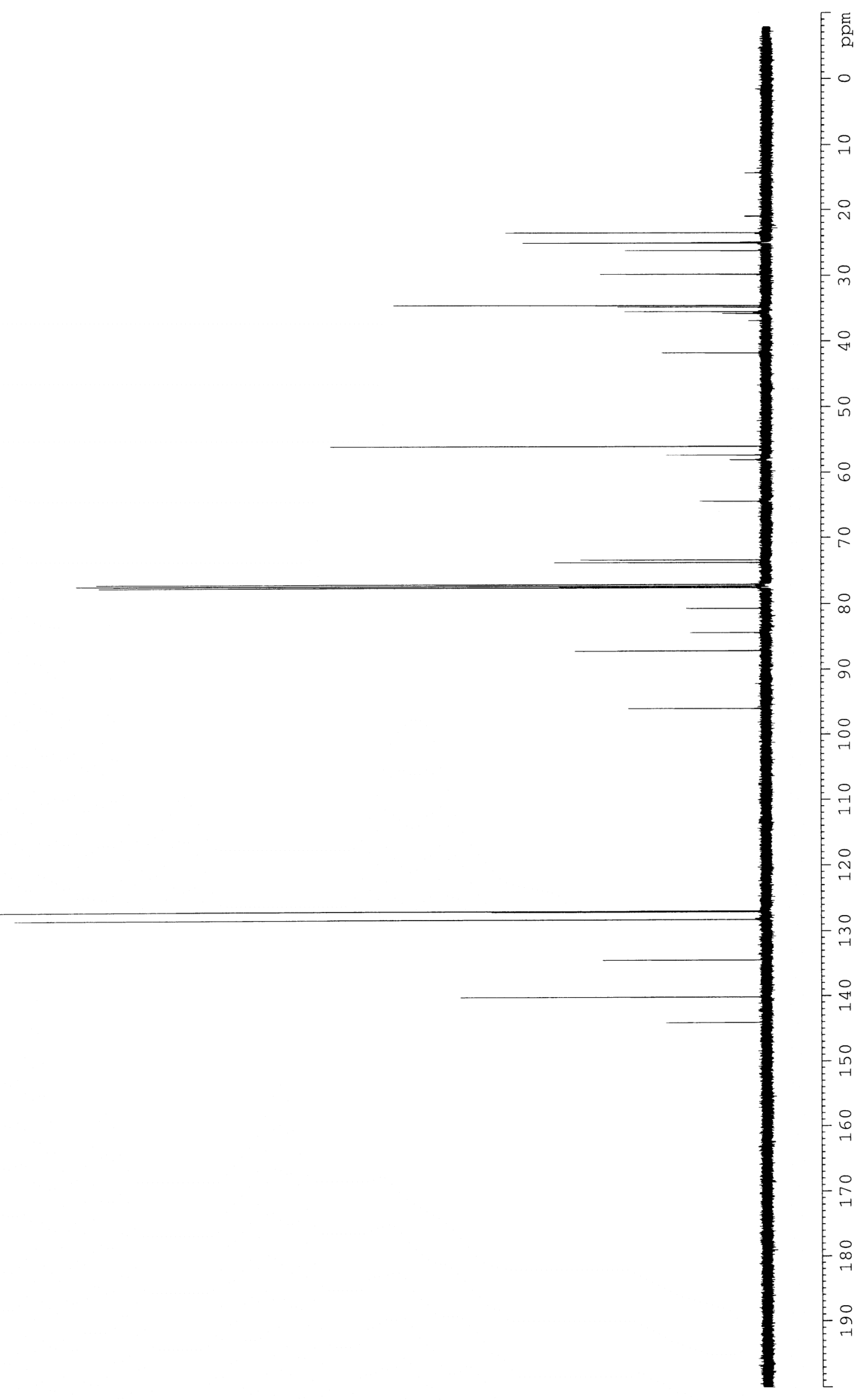




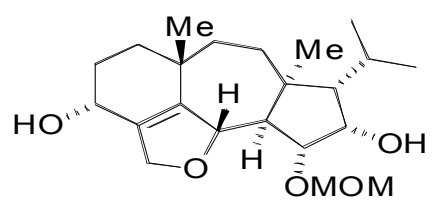

38

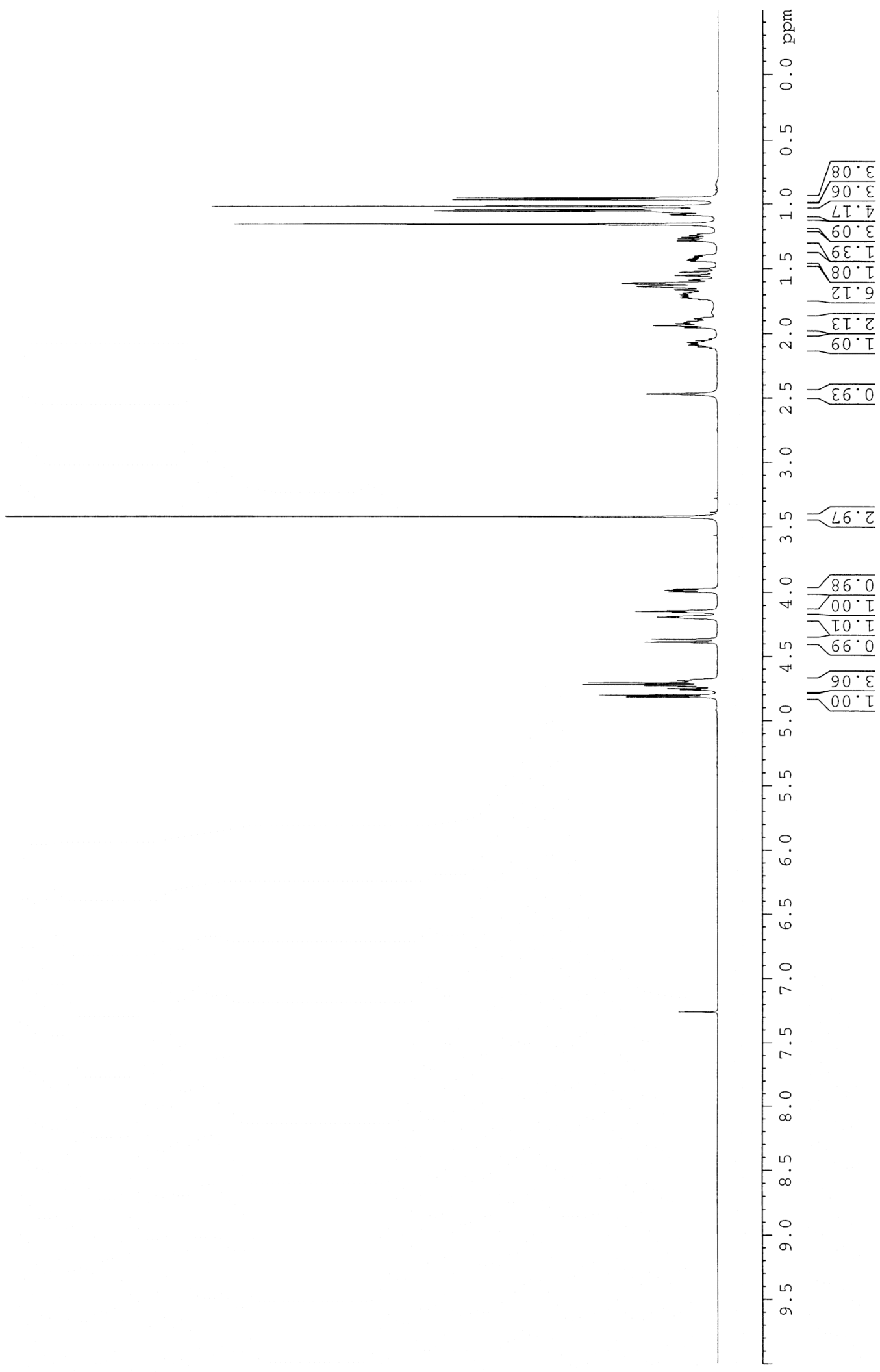




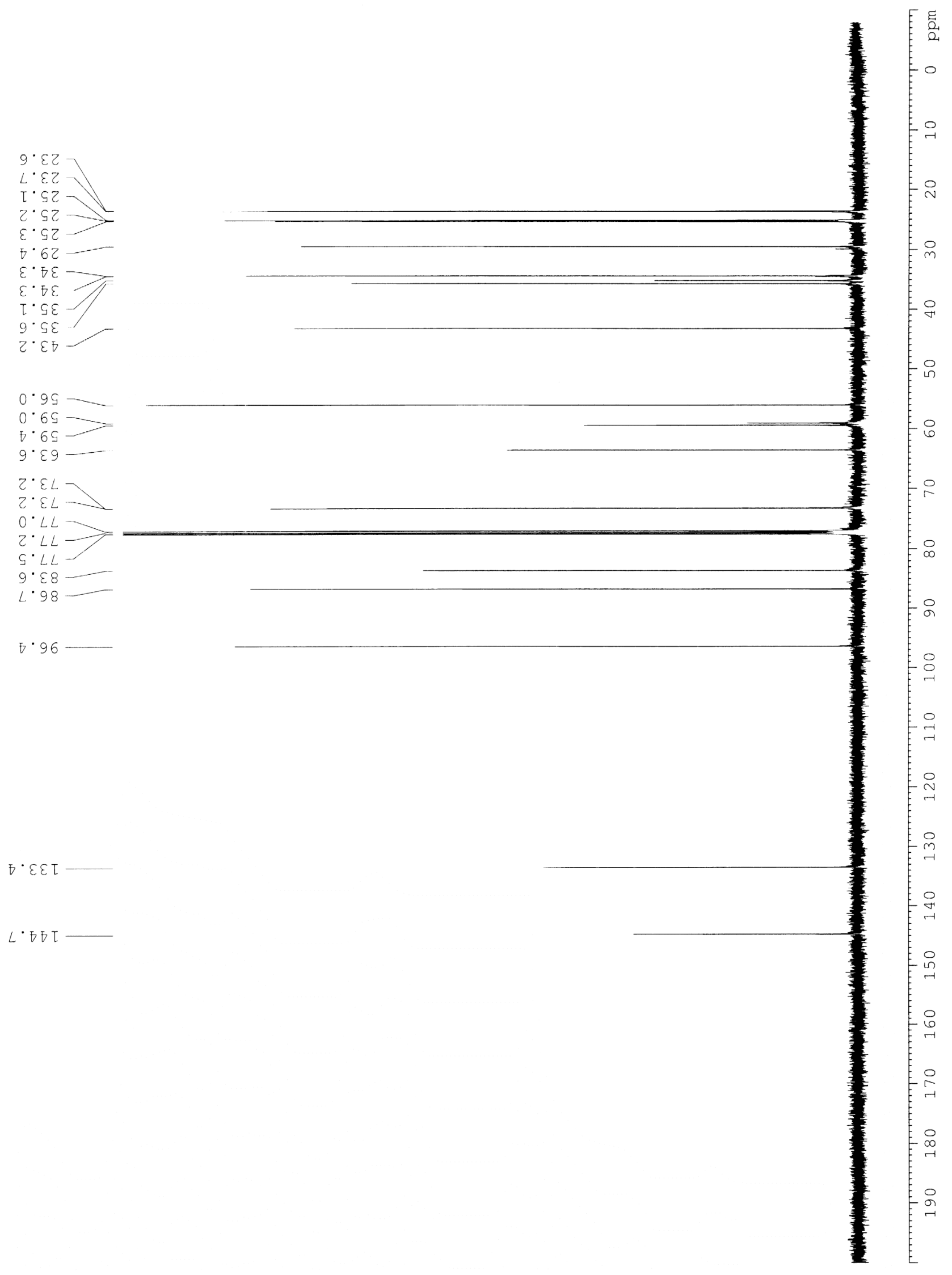




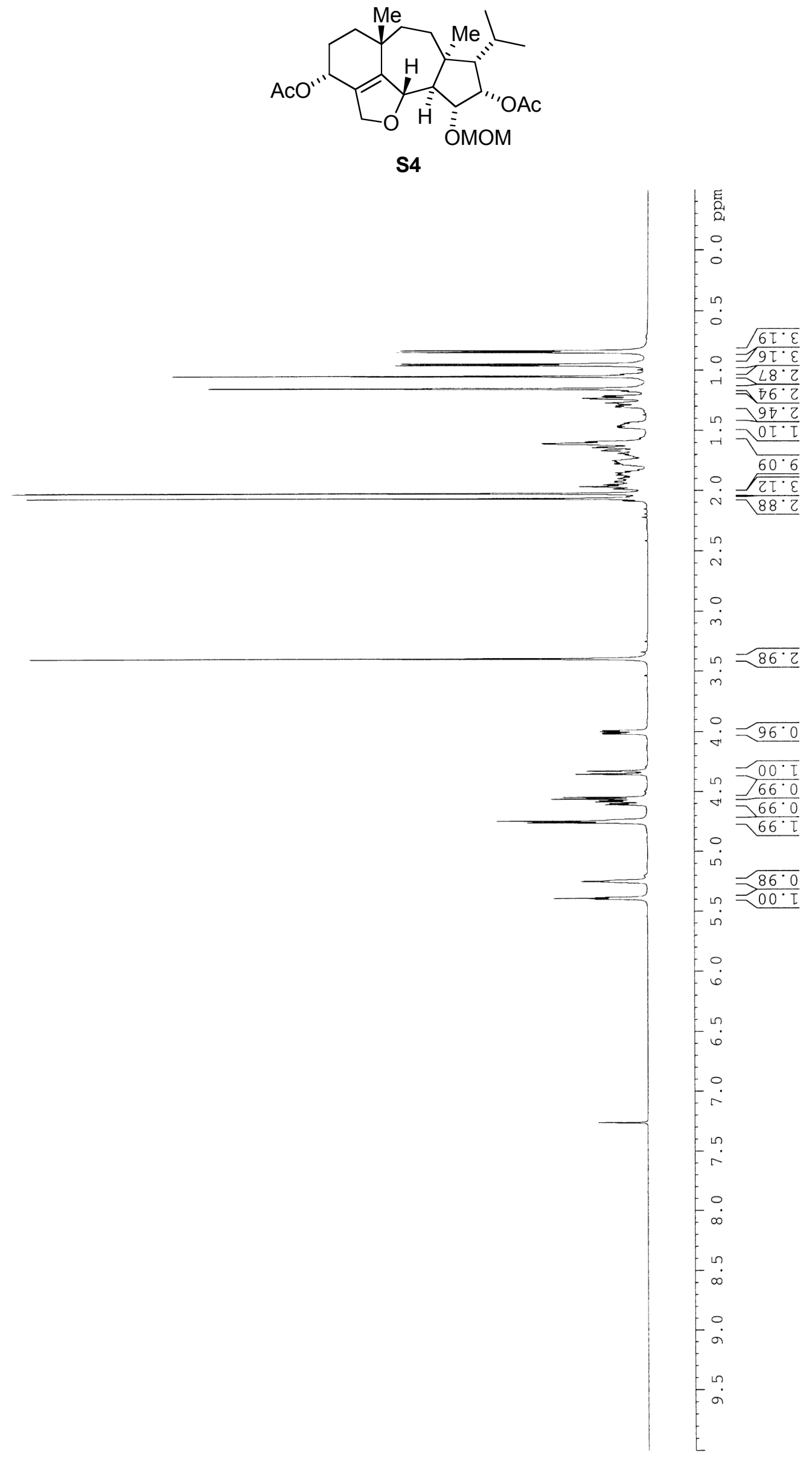




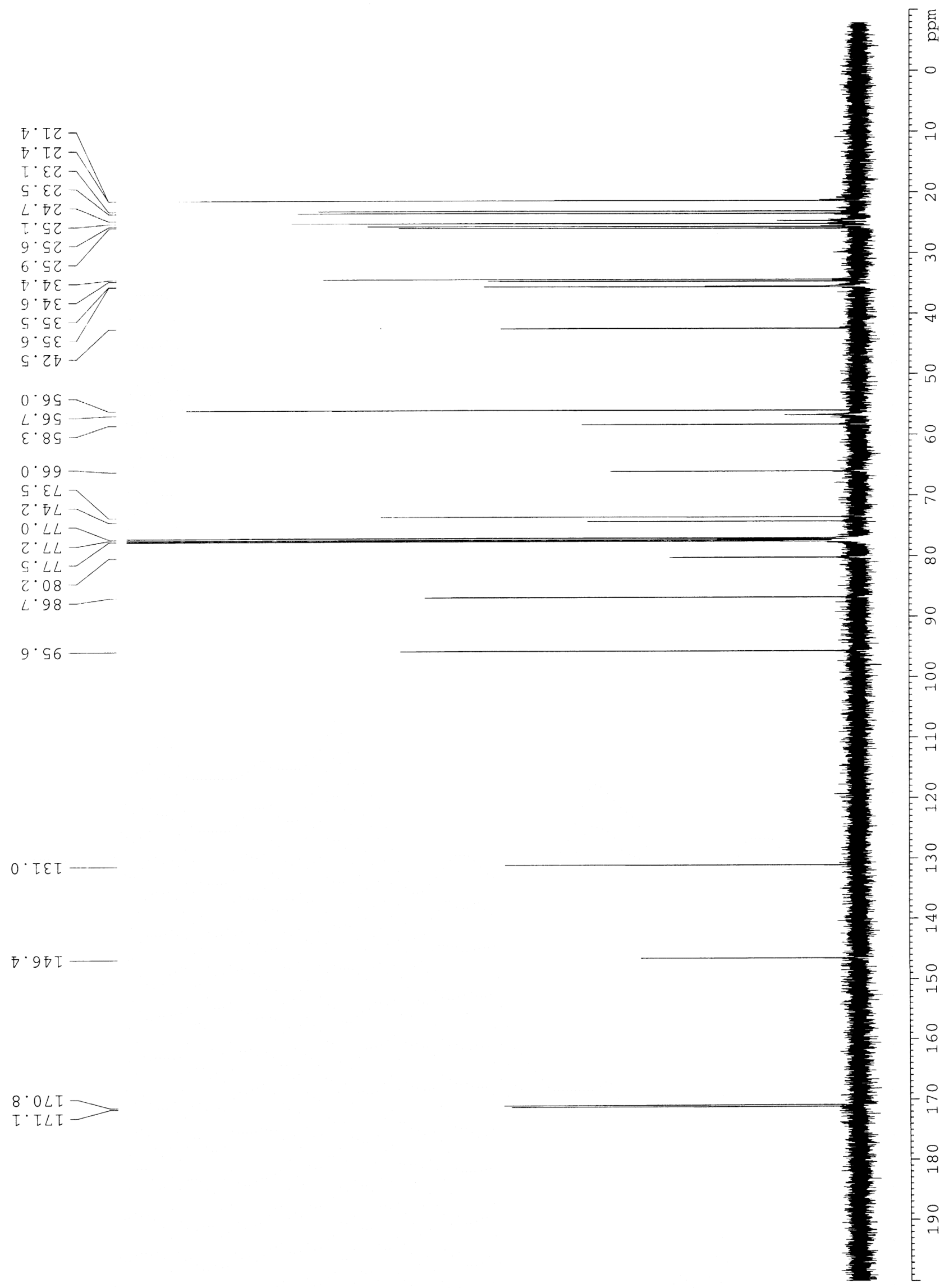




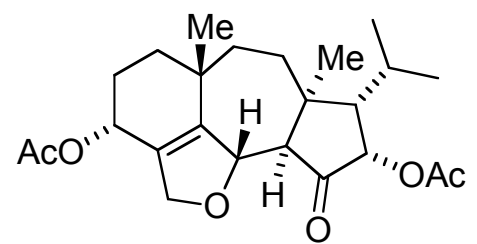

(-)-heptemerone B

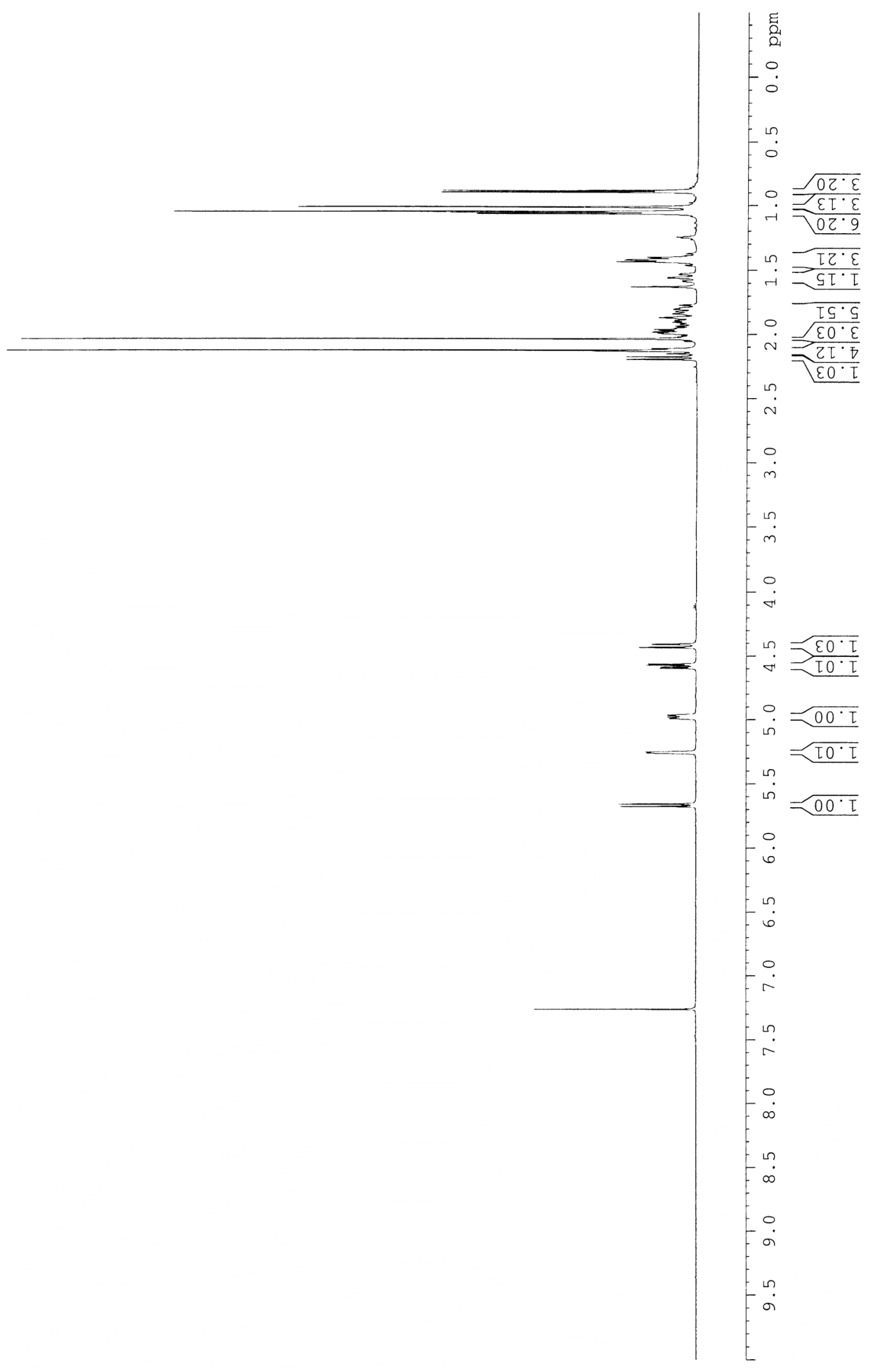


(-)-heptemerone B
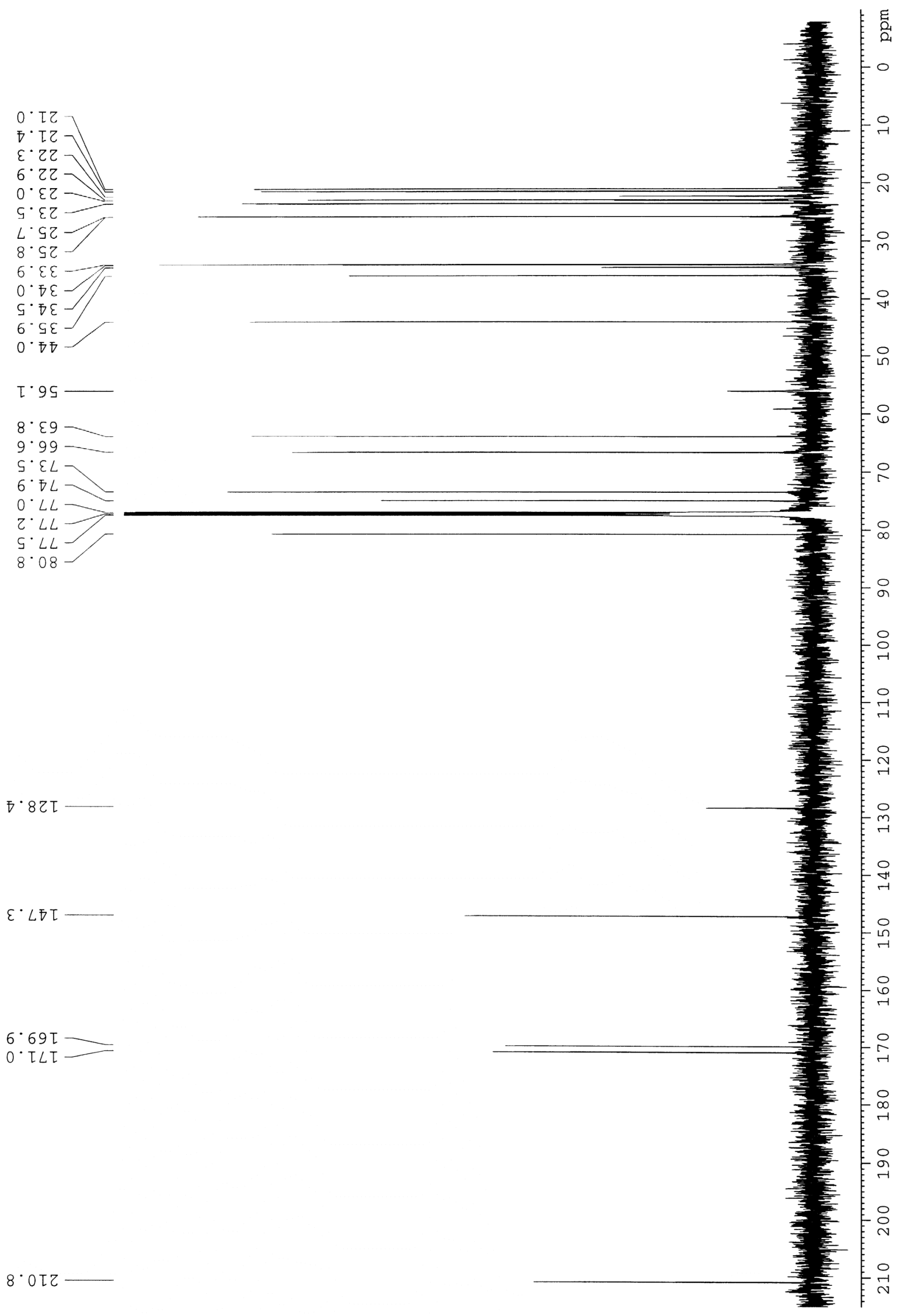


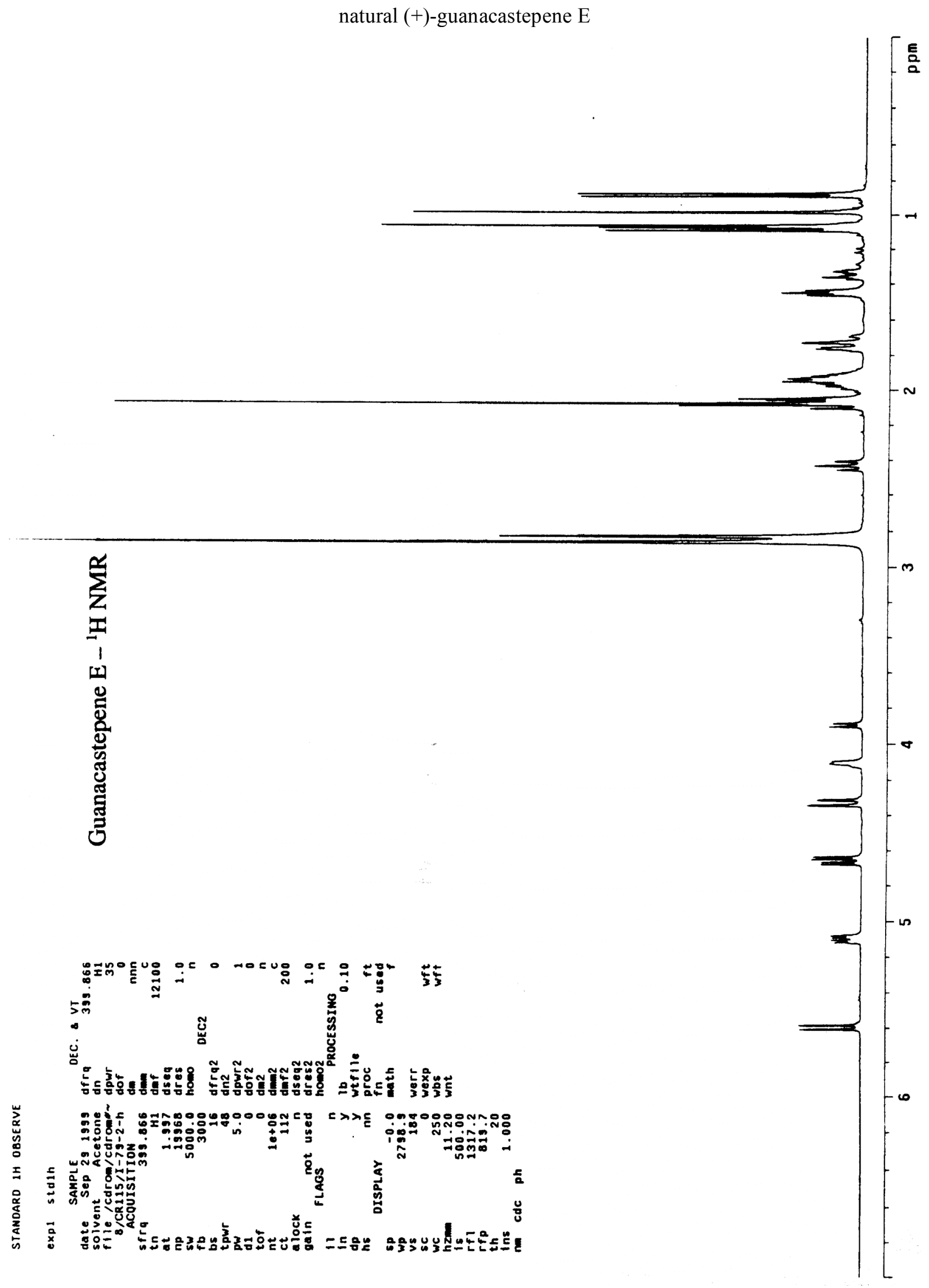


synthetic (-)-guanacastepene E

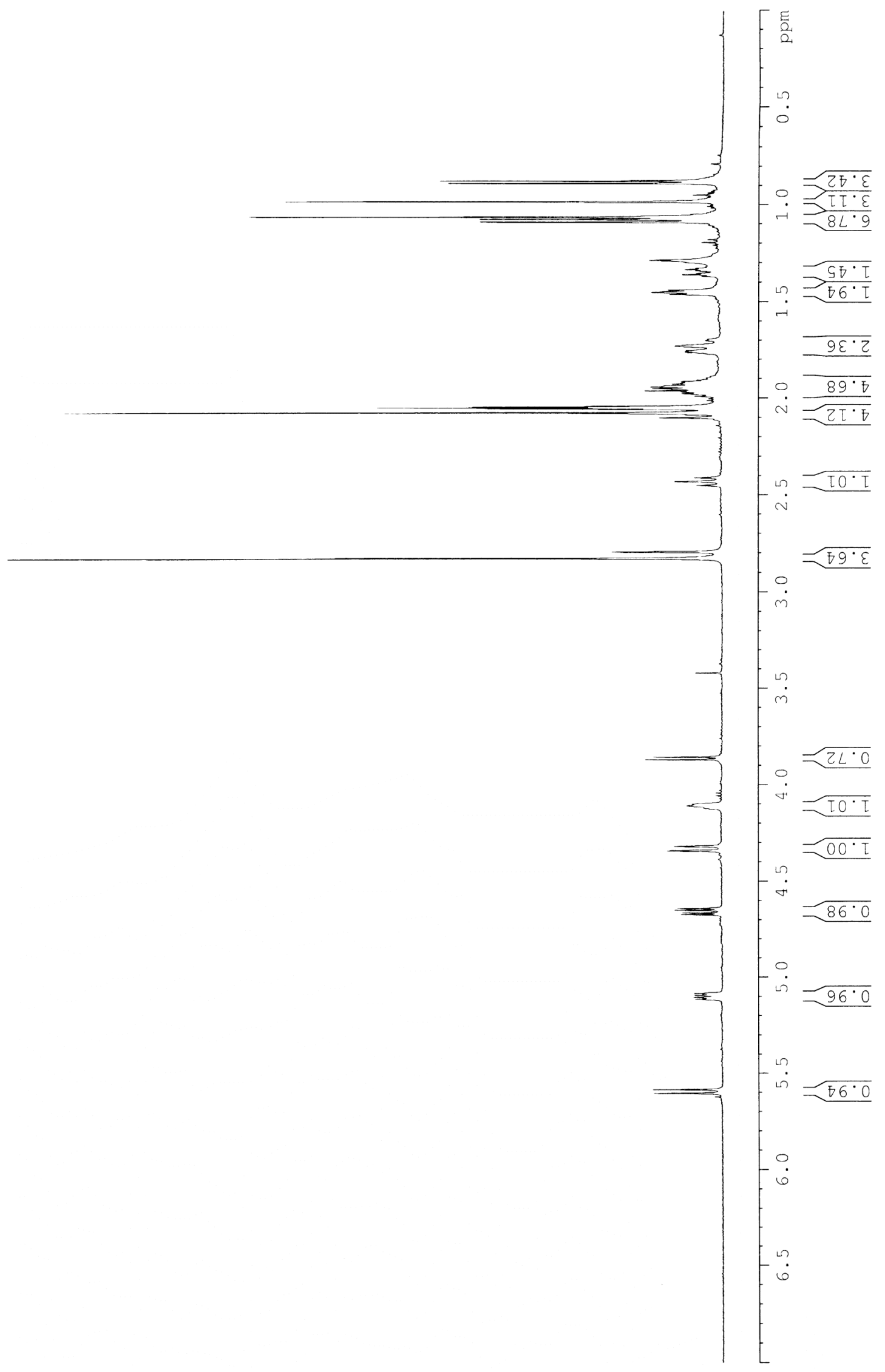


natural (+)-guanacastepene E

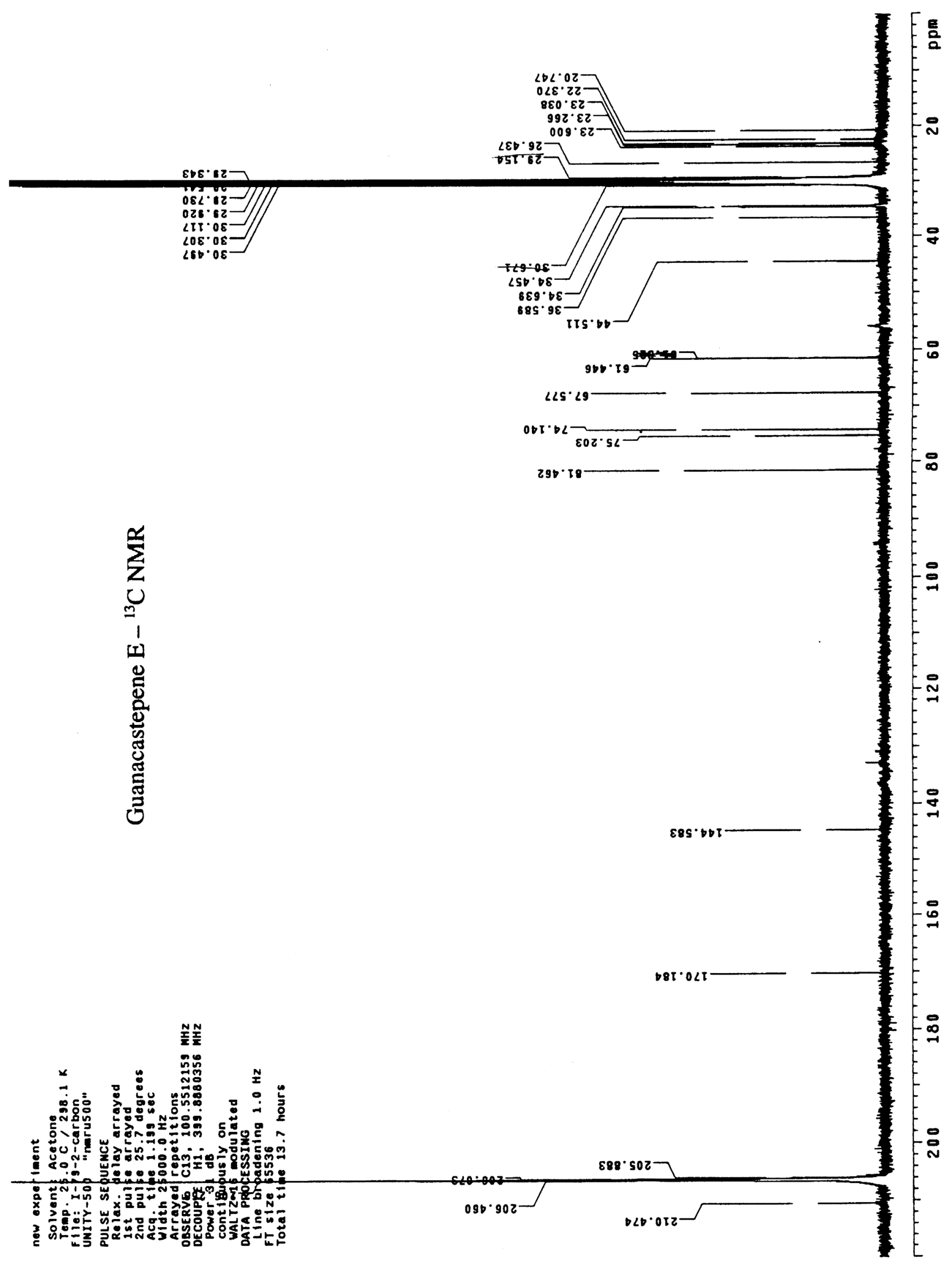


synthetic (-)-guanacastepene E

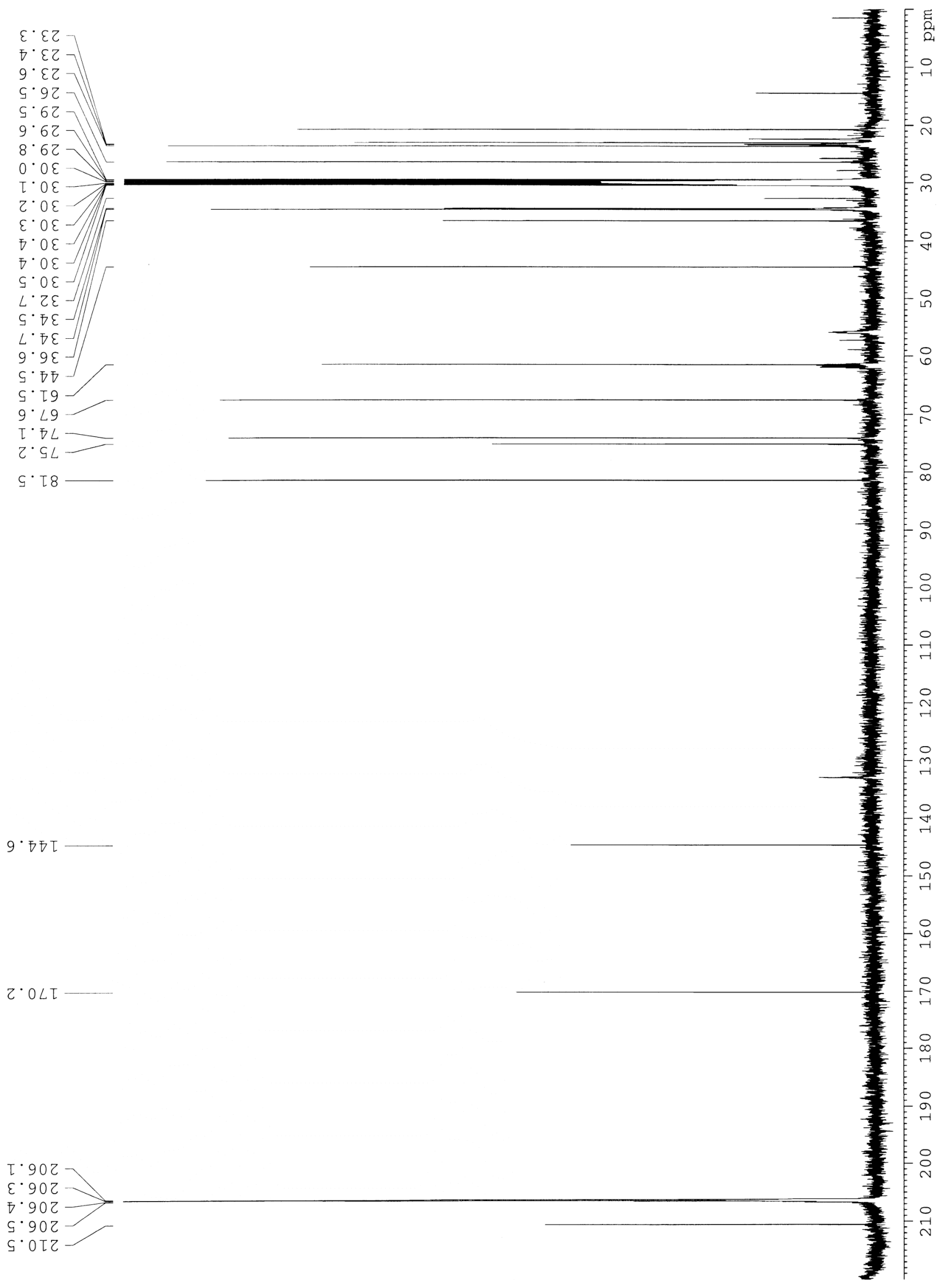


X-ray crystallographic data for compound $\mathbf{1 3} \cdot \mathrm{NMP}_{2}$
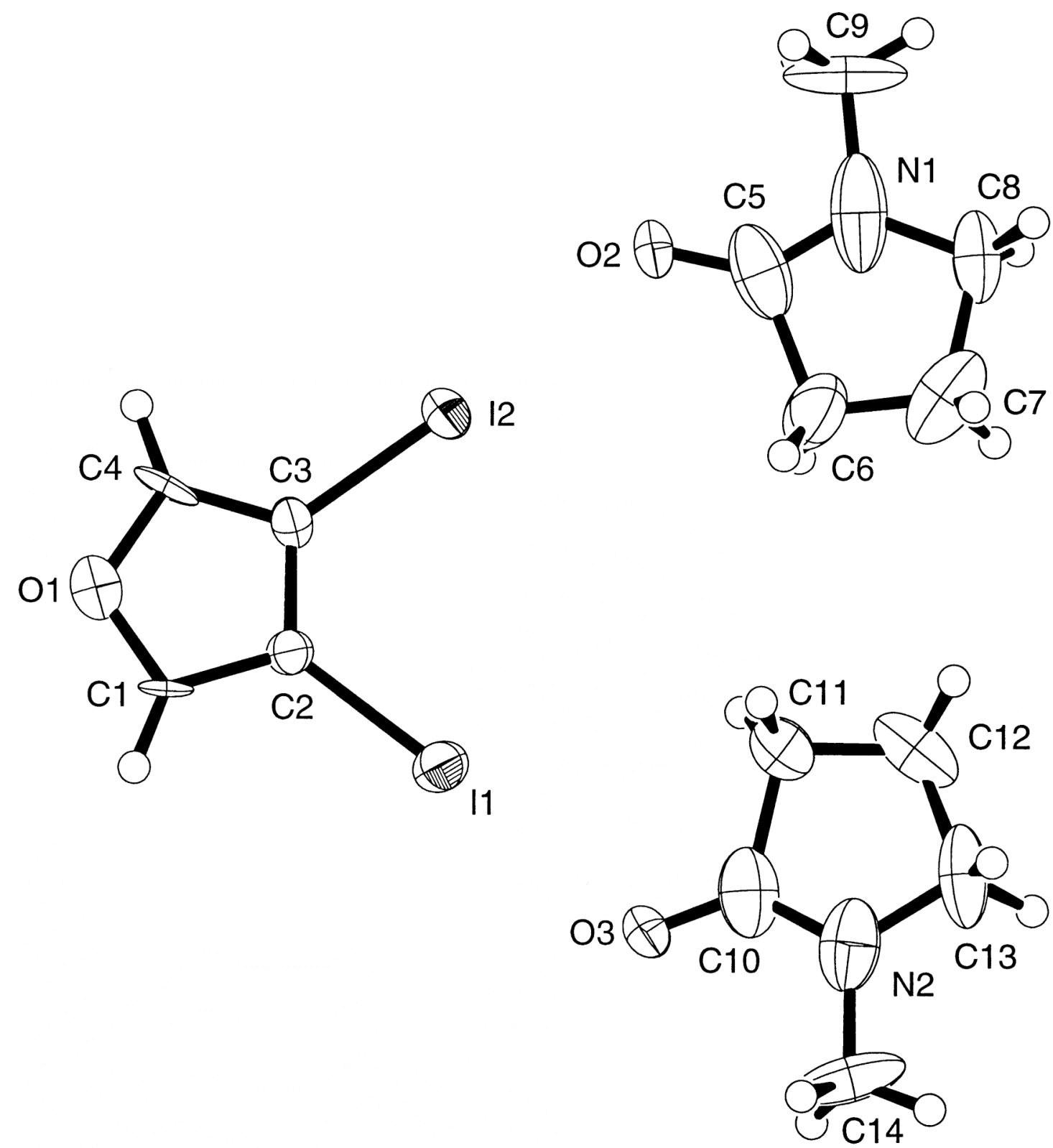
A. Crystal Data

\author{
Empirical Formula \\ Formula Weight \\ Crystal Color, Habit \\ Crystal Dimensions \\ Crystal System \\ Lattice Type \\ Lattice Parameters
}

Space Group

$Z$ value

$\mathrm{D}_{\text {calc }}$

$\mathrm{F}_{000}$

$\mu(\operatorname{Mok} \alpha)$

Diffractometer

Radiation

Detector Position

Exposure Time

Scan Type

$2 \theta_{\max }$

No. of Reflections Measured

Corrections

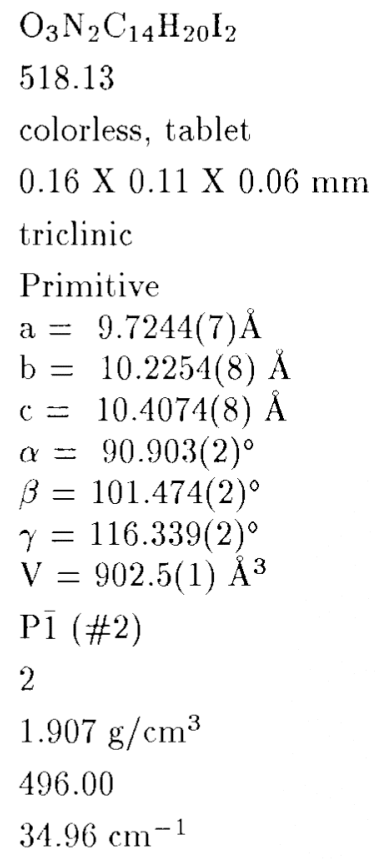

13. Intensity Measurements

\section{Bruker SMART CCD}

$\operatorname{MoK} \alpha(\lambda=0.71069 \AA)$ graphite monochromated $60.00 \mathrm{~mm}$

10.0 seconds per frame.

$\omega(0.3$ degrees per frame) $49.4^{\circ}$

Total: 4557

Unique: $2861\left(\mathrm{R}_{\text {int }}=0.044\right)$

Lorentz-polarization

Absorption $(\operatorname{Tmax}=0.95 \mathrm{Tmin}=0.64)$

C. Structure Solution and Refinement

Structure Solution

Refinement

Function Minimized

Least Squares Weights

p-factor
Direct Methods (SIR97)

Full-matrix least-squares

$\Sigma w(|F o|-|F c|)^{2}$

$w=\frac{1}{\sigma^{2}(F o)}=\left[\sigma_{c}^{2}(F o)+\frac{p^{2}}{4} F o^{2}\right]^{-1}$

0.0300 
Anomalous Dispersion

No. Observations (I>3.00 $\sigma(\mathrm{I})$ )

No. Variables

Reflection/Parameter Ratio

Residuals: R; Rw; Rall

Goodness of Fit Indicator

Max Shift/Error in Final Cycle

Maximum peak in Final Diff. Map

Minimum peak in Final Diff. Map
All non-hydrogen atoms

2038

190

10.73

$0.043 ; 0.054 ; 0.067$

1.43

0.01

$0.85 e^{-/ \AA^{3}}$

$-1.06 e^{-} / \AA^{3}$ 
Table 1. Atomic coordinates and $\mathrm{B}_{i s o} / \mathrm{B}_{\varepsilon q}$

\begin{tabular}{|c|c|c|c|c|}
\hline atom & $\mathrm{x}$ & $\mathrm{y}$ & $z$ & $\mathrm{~B}_{e q}$ \\
\hline $\mathrm{I}(1)$ & $0.33927(7)$ & $0.05798(7)$ & $-0.13400(6)$ & $2.96(2)$ \\
\hline $\mathrm{I}(2)$ & $0.34173(7)$ & $0.32296(7)$ & $-0.40130(6)$ & $2.81(2)$ \\
\hline $\mathrm{O}(1)$ & $0.7840(7)$ & $0.3506(7)$ & $-0.2047(7)$ & $3.4(2)$ \\
\hline $\mathrm{O}(2)$ & $0.0801(7)$ & $0.3533(8)$ & $-0.5631(7)$ & $3.9(2)$ \\
\hline $\mathrm{O}(3)$ & $0.0719(8)$ & $-0.1508(8)$ & $-0.0427(7)$ & $3.9(2)$ \\
\hline$N(1)$ & $-0.181(1)$ & $0.289(1)$ & $-0.627(1)$ & $6.9(3)$ \\
\hline$N(2)$ & $-0.191(1)$ & $-0.268(1)$ & $-0.079(1)$ & $5.1(3)$ \\
\hline $\mathrm{C}(1)$ & $0.692(1)$ & $0.2400(9)$ & $-0.1428(7)$ & $1.6(2)$ \\
\hline $\mathrm{C}(2)$ & $0.535(1)$ & $0.2110(10)$ & $-0.1927(9)$ & $2.2(2)$ \\
\hline $\mathrm{C}(3)$ & $0.5366(10)$ & $0.3106(10)$ & $-0.2905(9)$ & $2.3(2)$ \\
\hline$C(4)$ & $0.698(1)$ & $0.396(1)$ & $-0.2945(9)$ & $2.6(2)$ \\
\hline $\mathrm{C}(5)$ & $-0.056(1)$ & $0.293(1)$ & $-0.562(1)$ & $5.4(4)$ \\
\hline$C(6)$ & $-0.128(1)$ & $0.177(1)$ & $-0.458(1)$ & $5.5(4)$ \\
\hline$C(7)$ & $-0.297(2)$ & $0.118(1)$ & $-0.499(2)$ & $6.7(4)$ \\
\hline $\mathrm{C}(8)$ & $-0.330(1)$ & $0.216(1)$ & $-0.607(1)$ & $4.9(3)$ \\
\hline $\mathrm{C}(9)$ & $-0.162(2)$ & $0.389(2)$ & $-0.735(1)$ & $7.3(4)$ \\
\hline $\mathrm{C}(10)$ & $-0.060(1)$ & $-0.178(1)$ & $-0.097(1)$ & $4.7(3)$ \\
\hline$C(11)$ & $-0.100(1)$ & $-0.092(1)$ & $-0.215(1)$ & $4.9(3)$ \\
\hline $\mathrm{C}(12)$ & $-0.267(2)$ & $-0.135(2)$ & $-0.227(1)$ & $5.6(4)$ \\
\hline $\mathrm{C}(13)$ & $-0.337(1)$ & $-0.284(1)$ & $-0.162(1)$ & $5.4(3)$ \\
\hline $\mathrm{C}(14)$ & $-0.194(2)$ & $-0.372(1)$ & $0.021(1)$ & $6.3(1)$ \\
\hline $\mathrm{H}(1)$ & 0.7304 & 0.1932 & -0.0794 & 2.0976 \\
\hline $\mathrm{H}(2)$ & 0.7378 & 0.4710 & -0.3491 & 3.0731 \\
\hline $\mathrm{H}(3)$ & -0.0992 & 0.1011 & -0.4555 & 6.2069 \\
\hline $\mathrm{H}(4)$ & -0.0952 & 0.2276 & -0.3681 & 0.2069 \\
\hline $\mathrm{H}(5)$ & -0.3452 & 0.0225 & -0.5460 & 8.5815 \\
\hline $\mathrm{II}(6)$ & -0.3485 & 0.1223 & -0.4341 & 8.5815 \\
\hline $\mathrm{H}(7)$ & -0.3688 & 0.2776 & -0.5698 & 5.8217 \\
\hline $\mathrm{H}(8)$ & -0.4116 & 0.1556 & $-(0.6826$ & 5.8217 \\
\hline $\mathrm{H}(9)$ & -0.0878 & 0.4896 & -0.6960 & 6.5831 \\
\hline $\mathrm{II}(10)$ & -0.1078 & 0.3639 & -0.7950 & 6.5831 \\
\hline $\mathrm{H}(11)$ & -0.2533 & 0.3848 & -0.7821 & 6.5831 \\
\hline $\mathrm{H}(12)$ & -0.0361 & 0.0105 & -0.1980 & 5.6997 \\
\hline $\mathrm{H}(13)$ & -0.0861 & -0.1263 & -0.2971 & 5.6997 \\
\hline $\mathrm{H}(14)$ & -0.2761 & -0.0596 & -0.1747 & 6.3193 \\
\hline $\mathrm{H}(15)$ & -0.3218 & -0.1441 & -0.3139 & 6.3193 \\
\hline $\mathrm{H}(16)$ & -0.3871 & -0.3681 & -0.2301 & 6.3830 \\
\hline $\mathrm{H}(17)$ & -0.4163 & -0.2914 & -0.1161 & 6.3830 \\
\hline $\mathrm{H}(18)$ & -0.2978 & -0.4346 & 0.0278 & 6.1038 \\
\hline
\end{tabular}


Table 1. Atomic coordinates and $\mathrm{B}_{i s o} / \mathrm{B}_{e q}$ (continued)

$\begin{array}{lcccl}\text { atom } & \mathrm{x} & \mathrm{y} & \mathrm{z} & \mathrm{B}_{e q} \\ \mathrm{H}(19) & -0.1449 & -0.4297 & -0.0005 & 6.1038 \\ \mathrm{H}(20) & -0.1344 & -0.3169 & 0.1073 & 6.1038\end{array}$

$$
B_{e q}=\frac{8}{3} \pi^{2}\left(U_{11}\left(a a^{*}\right)^{2}+U_{22}\left(b b^{*}\right)^{2}+U_{33}\left(c c^{*}\right)^{2}+2 U_{12} a a^{*} b b^{*} \cos \gamma+2 U_{13} a a^{*} c c^{*} \cos \beta+2 U_{23} b b^{*} c c^{*} \cos \alpha\right)
$$

Table 2. Anisotropic Displacement Parameters

\begin{tabular}{llllllr} 
atom & $\mathrm{U}_{11}$ & $\mathrm{U}_{22}$ & $\mathrm{U}_{33}$ & $\mathrm{U}_{12}$ & $\mathrm{U}_{13}$ & \multicolumn{1}{c}{$\mathrm{U}_{23}$} \\
$\mathrm{I}(1)$ & $0.0387(4)$ & $0.0363(4)$ & $0.0368(4)$ & $0.0147(3)$ & $0.0119(3)$ & $0.0091(3)$ \\
$\mathrm{I}(2)$ & $0.0295(4)$ & $0.0391(4)$ & $0.0354(4)$ & $0.0157(3)$ & $0.0014(3)$ & $0.0070(3)$ \\
$\mathrm{O}(1)$ & $0.033(4)$ & $0.047(4)$ & $0.047(4)$ & $0.019(4)$ & $0.004(3)$ & $-0.001(4)$ \\
$\mathrm{O}(2)$ & $0.020(3)$ & $0.063(5)$ & $0.061(5)$ & $0.015(3)$ & $0.005(3)$ & $0.025(4)$ \\
$\mathrm{O}(3)$ & $0.027(4)$ & $0.058(5)$ & $0.053(5)$ & $0.014(4)$ & $0.000(3)$ & $0.025(4)$ \\
$\mathrm{N}(1)$ & $0.038(6)$ & $0.075(8)$ & $0.13(1)$ & $0.015(6)$ & $0.001(6)$ & $-0.053(8)$ \\
$\mathrm{N}(2)$ & $0.047(6)$ & $0.065(7)$ & $0.079(8)$ & $0.020(6)$ & $0.019(6)$ & $-0.022(6)$ \\
$\mathrm{C}(1)$ & $0.038(5)$ & $0.020(5)$ & $0.007(4)$ & $0.016(4)$ & $0.005(4)$ & $0.008(4)$ \\
$\mathrm{C}(2)$ & $0.030(5)$ & $0.032(5)$ & $0.031(5)$ & $0.019(5)$ & $0.012(4)$ & $0.009(4)$ \\
$\mathrm{C}(3)$ & $0.023(5)$ & $0.032(5)$ & $0.033(6)$ & $0.013(4)$ & $0.004(4)$ & $0.003(4)$ \\
$\mathrm{C}(4)$ & $0.052(6)$ & $0.037(6)$ & $0.022(5)$ & $0.036(5)$ & $-0.007(4)$ & $0.006(4)$ \\
$\mathrm{C}(5)$ & $0.050(8)$ & $0.066(9)$ & $0.08(1)$ & $0.034(7)$ & $-0.013(7)$ & $-0.030(8)$ \\
$\mathrm{C}(6)$ & $0.056(8)$ & $0.077(10)$ & $0.088(10)$ & $0.031(7)$ & $0.039(7)$ & $0.023(8)$ \\
$\mathrm{C}(7)$ & $0.08(1)$ & $0.058(9)$ & $0.11(1)$ & $0.014(8)$ & $0.054(9)$ & $0.003(9)$ \\
$\mathrm{C}(8)$ & $0.030(6)$ & $0.068(8)$ & $0.083(10)$ & $0.022(6)$ & $0.008(6)$ & $-0.019(7)$ \\
$\mathrm{C}(9)$ & $0.19(2)$ & $0.065(9)$ & $0.043(7)$ & $0.07(1)$ & $0.036(9)$ & $0.035(7)$ \\
$\mathrm{C}(10)$ & $0.043(7)$ & $0.053(8)$ & $0.069(9)$ & $0.014(6)$ & $0.000(7)$ & $-0.027(7)$ \\
$\mathrm{C}(11)$ & $0.051(7)$ & $0.082(9)$ & $0.046(7)$ & $0.027(7)$ & $0.000(6)$ & $0.014(7)$ \\
$\mathrm{C}(12)$ & $0.089(10)$ & $0.084(10)$ & $0.053(8)$ & $0.062(9)$ & $-0.014(7)$ & $-0.005(7)$ \\
$\mathrm{C}(13)$ & $0.029(6)$ & $0.070(9)$ & $0.090(10)$ & $0.014(6)$ & $-0.003(6)$ & $-0.037(8)$ \\
$\mathrm{C}(14)$ & $0.15(1)$ & $0.033(7)$ & $0.056(8)$ & $0.033(8)$ & $0.051(9)$ & $0.022(6)$
\end{tabular}

The general temperature factor expression:

$$
\exp \left(-2 \pi^{2}\left(a^{* 2} U_{11} h^{2}+b^{* 2} U_{22} k^{2}+c^{* 2} U_{33} l^{2}+2 a^{*} b^{*} U_{12} h k+2 a^{*} c^{*} U_{13} h l+2 b^{*} c^{*} U_{23} k l\right)\right)
$$


Table 3. Bond Lengths $(\AA)$

$\begin{array}{llllll}\text { atom } & \text { atom } & \text { distance } & \text { atom } & \text { atom } & \text { distance } \\ \mathrm{I} 1 & \mathrm{C} 2 & 2.074(8) & \mathrm{I} 2 & \mathrm{C} 3 & 2.070(9) \\ \mathrm{O} 1 & \mathrm{C} 1 & 1.358(10) & \mathrm{O} 1 & \mathrm{C} 4 & 1.36(1) \\ \mathrm{O} 2 & \mathrm{C} 5 & 1.19(1) & \mathrm{O} 3 & \mathrm{C} 10 & 1.19(1) \\ \mathrm{N} 1 & \mathrm{C} 5 & 1.25(2) & \mathrm{N} 1 & \mathrm{C} 8 & 1.37(1) \\ \mathrm{N} 1 & \mathrm{C} 9 & 1.51(2) & \mathrm{N} 2 & \mathrm{C} 10 & 1.25(1) \\ \mathrm{N} 2 & \mathrm{C} 13 & 1.45(2) & \mathrm{N} 2 & \mathrm{C} 14 & 1.50(2) \\ \mathrm{C} 1 & \mathrm{C} 2 & 1.40(1) & \mathrm{C} 2 & \mathrm{C} 3 & 1.45(1) \\ \mathrm{C} 3 & \mathrm{C} 4 & 1.42(1) & \mathrm{C} 5 & \mathrm{C} 6 & 1.64(2) \\ \mathrm{C} 6 & \mathrm{C} 7 & 1.44(2) & \mathrm{C} 7 & \mathrm{C} 8 & 1.60(2) \\ \mathrm{C} 10 & \mathrm{C} 11 & 1.61(2) & \mathrm{C} 11 & \mathrm{C} 12 & 1.47(2) \\ \mathrm{C} 12 & \mathrm{C} 13 & 1.59(2) & & & \end{array}$

Table 4. Bond Lengths $(\AA)$

$\begin{array}{llllll}\text { atom } & \text { atom } & \text { distance } & \text { atom } & \text { atom } & \text { distance } \\ \text { C1 } & \text { H1 } & 0.93 & \text { C4 } & \text { H2 } & 0.95 \\ \text { C6 } & \text { H3 } & 0.93 & \text { C6 } & \text { H4 } & 0.98 \\ \text { C7 } & \text { H5 } & 0.94 & \mathrm{C} 7 & \text { H6 } & 0.94 \\ \text { C8 } & \text { H7 } & 0.97 & \mathrm{C} 8 & \text { H8 } & 0.97 \\ \text { C9 } & \text { H9 } & 0.97 & \mathrm{C} 9 & \text { H10 } & 0.99 \\ \text { C9 } & \text { H11 } & 0.91 & \mathrm{C} 11 & \text { H12 } & 0.94 \\ \text { C11 } & \text { H13 } & 0.97 & \mathrm{C} 12 & \text { H14 } & 0.98 \\ \text { C12 } & \text { H15 } & 0.93 & \mathrm{C} 13 & \text { H16 } & 0.98 \\ \text { C13 } & \text { H17 } & 0.96 & \mathrm{C} 14 & \text { H18 } & 0.94 \\ \text { C14 } & \text { H19 } & 0.95 & \mathrm{C} 14 & \text { H20 } & 0.97\end{array}$

Table 5. Bond Angles $\left({ }^{\circ}\right)$

$\begin{array}{llllllll}\text { atom } & \text { atom } & \text { atom } & \text { angle } & \text { atom } & \text { atom } & \text { atom } & \text { angle } \\ \mathrm{C} 1 & \mathrm{O} 1 & \mathrm{C} 4 & 112.2(7) & \mathrm{C} 5 & \mathrm{~N} 1 & \mathrm{C} 8 & 128(1) \\ \mathrm{C} 5 & \mathrm{~N} 1 & \mathrm{C} 9 & 115(1) & \mathrm{C} 8 & \mathrm{~N} 1 & \mathrm{C} 9 & 115(1) \\ \mathrm{C} 10 & \mathrm{~N} 2 & \mathrm{C} 13 & 121(1) & \mathrm{C} 10 & \mathrm{~N} 2 & \mathrm{C} 14 & 118(1) \\ \mathrm{C} 13 & \mathrm{~N} 2 & \mathrm{C} 14 & 119(1) & \mathrm{O} 1 & \mathrm{C} 1 & \mathrm{C} 2 & 107.6(7) \\ \mathrm{I} 1 & \mathrm{C} 2 & \mathrm{C} 1 & 126.4(6) & \mathrm{I} 1 & \mathrm{C} 2 & \mathrm{C} 3 & 126.6(6) \\ \mathrm{C} 1 & \mathrm{C} 2 & \mathrm{C} 3 & 107.0(7) & \mathrm{I} 2 & \mathrm{C} 3 & \mathrm{C} 2 & 126.2(6) \\ \mathrm{I} 2 & \mathrm{C} 3 & \mathrm{C} 4 & 127.7(7) & \mathrm{C} 2 & \mathrm{C} 3 & \mathrm{C} 4 & 106.1(7) \\ \mathrm{O} 1 & \mathrm{C} 4 & \mathrm{C} 3 & 107.1(8) & \mathrm{O} 2 & \mathrm{C} 5 & \mathrm{~N} 1 & 136(1) \\ \mathrm{O} 2 & \mathrm{C} 5 & \mathrm{C} 6 & 123(1) & \mathrm{N} 1 & \mathrm{C} 5 & \mathrm{C} 6 & 99(1) \\ \mathrm{C} 5 & \mathrm{C} 6 & \mathrm{C} 7 & 105(1) & \mathrm{C} 6 & \mathrm{C} 7 & \mathrm{C} 8 & 106(1) \\ \mathrm{N} 1 & \mathrm{C} 8 & \mathrm{C} 7 & 97(1) & \mathrm{O} 3 & \mathrm{C} 10 & \mathrm{~N} 2 & 131(1) \\ \mathrm{O} 3 & \mathrm{C} 10 & \mathrm{C} 11 & 123(1) & \mathrm{N} 2 & \mathrm{C} 10 & \mathrm{C} 11 & 105(1) \\ \mathrm{C} 10 & \mathrm{C} 11 & \mathrm{C} 12 & 103(1) & \mathrm{C} 11 & \mathrm{C} 12 & \mathrm{C} 13 & 106(1) \\ \mathrm{N} 2 & \mathrm{C} 13 & \mathrm{C} 12 & 98.1(9) & & & & \end{array}$


Table 6. Bond Angles $\left({ }^{\circ}\right)$

\begin{tabular}{|c|c|c|c|c|c|c|c|}
\hline atom & atom & atom & angle & atom & atom & atom & angle \\
\hline O1 & $\mathrm{C} 1$ & $\mathrm{H} 1$ & 124.7 & $\mathrm{C} 2$ & $\mathrm{C} 1$ & H1 & 127.8 \\
\hline O1 & $\mathrm{C} 4$ & $\mathrm{H} 2$ & 126.0 & C3 & $\mathrm{C} 4$ & $\mathrm{H} 2$ & 126.9 \\
\hline C5 & $\mathrm{C} 6$ & H3 & 114.0 & $\mathrm{C} 5$ & $\mathrm{C} 6$ & $\mathrm{H} 4$ & 110.8 \\
\hline $\mathrm{C} 7$ & C6 & H3 & 110.0 & $\mathrm{C} 7$ & C6 & $\mathrm{II} 4$ & 107.7 \\
\hline H3 & $\mathrm{C} 6$ & $\mathrm{H} 4$ & 108.6 & $\mathrm{C} 6$ & $\mathrm{C} 7$ & H5 & 112.9 \\
\hline C6 & $\mathrm{C} 7$ & $\mathrm{H} 6$ & 115.9 & $\mathrm{C} 8$ & $\mathrm{C} 7$ & $\mathrm{H} 5$ & 104.0 \\
\hline $\mathrm{C} 8$ & $\mathrm{C} 7$ & H6 & 105.2 & H5 & $\mathrm{C} 7$ & H6 & 111.1 \\
\hline N1 & $\mathrm{C} 8$ & $\mathrm{H} 7$ & 115.6 & N1 & $\mathrm{C} 8$ & 118 & 115.8 \\
\hline $\mathrm{C} 7$ & $\mathrm{C} 8$ & $\mathrm{H} 7$ & 110.2 & $\mathrm{C} 7$ & C8 & 118 & 110.9 \\
\hline $\mathrm{H} 7$ & $\mathrm{C} 8$ & II8 & 106.2 & N1 & C9 & $\mathrm{H} 9$ & 109.0 \\
\hline $\mathrm{N} 1$ & $\mathrm{C} 9$ & $\mathrm{H} 10$ & 108.0 & N1 & $\mathrm{C9}$ & 1111 & 114.4 \\
\hline H9 & $\mathrm{C} 9$ & H 10 & 104.5 & 119 & C9 & HI11 & 110.9 \\
\hline H 10 & $\mathrm{C9}$ & H11 & 109.6 & C10 & $\mathrm{C} 11$ & $\mathrm{H} 12$ & 112.4 \\
\hline C10 & C11 & II13 & 110.2 & C 12 & C.11 & II 12 & 113.1 \\
\hline C12 & C11 & II 13 & 109.3 & HI12 & C11 & HI13 & 108.5 \\
\hline C11 & $\mathrm{C} 12$ & H 14 & 108.4 & C11 & C.12 & H15 & 112.5 \\
\hline C13 & $\mathrm{C} 12$ & H14 & 108.3 & C13 & $\mathrm{C} 12$ & 1115 & 112.3 \\
\hline II 14 & $\mathrm{C} 12$ & 1115 & 108.5 & $\mathrm{~N} 2$ & C.13 & III6 & 114.0 \\
\hline $\mathrm{N} 2$ & C13 & $\mathrm{H} 17$ & 115.0 & $\mathrm{C} 12$ & C13 & H16 & 110.4 \\
\hline C12 & $\mathrm{C} 13$ & $H 17$ & 113.0 & 1116 & C:13 & $\mathrm{H} 17$ & 106.4 \\
\hline $\mathrm{N} 2$ & C14 & $\mathrm{H} 18$ & 111.4 & $\mathrm{~N} 2$ & Cl4 & 1119 & 110.6 \\
\hline $\mathrm{N} 2$ & $\mathrm{C} 14$ & $\mathrm{H} 20$ & 109.2 & II 18 & $\mathrm{C} 14$ & II19 & 109.9 \\
\hline 1118 & $\mathrm{C} 14$ & $\mathrm{H} 20$ & 108.3 & H19 & $\mathrm{C} 14$ & H 20 & 107.4 \\
\hline
\end{tabular}

Table 7. Torsion Angles $\left({ }^{\circ}\right)$

$\begin{array}{lllllllllc}\text { atom } & \text { atom } & \text { atom } & \text { atom } & \text { angle } & \text { atom } & \text { atom } & \text { atom } & \text { atom } & \text { angle } \\ \mathrm{I} 1 & \mathrm{C} 2 & \mathrm{C} 1 & \mathrm{O} 1 & -178.1(6) & \mathrm{I} 1 & \mathrm{C} 2 & \mathrm{C} 3 & \mathrm{I} 2 & -1(1) \\ \mathrm{I} 1 & \mathrm{C} 2 & \mathrm{C} 3 & \mathrm{C} 4 & 178.6(6) & \mathrm{I} 2 & \mathrm{C} 3 & \mathrm{C} 2 & \mathrm{C} 1 & 179.7(6) \\ \mathrm{I} 2 & \mathrm{C} 3 & \mathrm{C} 4 & \mathrm{O} 1 & 179.8(6) & \mathrm{O} 1 & \mathrm{C} 1 & \mathrm{C} 2 & \mathrm{C} 3 & 0.6(9) \\ \mathrm{O} 1 & \mathrm{C} 4 & \mathrm{C} 3 & \mathrm{C} 2 & 0(1) & \mathrm{O} 2 & \mathrm{C} 5 & \mathrm{~N} 1 & \mathrm{C} 8 & -176(1) \\ \mathrm{O} 2 & \mathrm{C} 5 & \mathrm{~N} 1 & \mathrm{C} 9 & -3(2) & \mathrm{O} 2 & \mathrm{C} 5 & \mathrm{C} 6 & \mathrm{C} 7 & -173(1) \\ \mathrm{O} 3 & \mathrm{C} 10 & \mathrm{~N} 2 & \mathrm{C} 13 & -177(1) & \mathrm{O} 3 & \mathrm{C} 10 & \mathrm{~N} 2 & \mathrm{C} 14 & -5(1) \\ \mathrm{O} 3 & \mathrm{C} 10 & \mathrm{C} 11 & \mathrm{C} 12 & -166(1) & \mathrm{N} 1 & \mathrm{C} 5 & \mathrm{C} 6 & \mathrm{C} 7 & 5(1) \\ \mathrm{N} 1 & \mathrm{C} 8 & \mathrm{C} 7 & \mathrm{C} 6 & 12(1) & \mathrm{N} 2 & \mathrm{C} 10 & \mathrm{C} 11 & \mathrm{C} 12 & 12(1) \\ \mathrm{N} 2 & \mathrm{C} 13 & \mathrm{C} 12 & \mathrm{C} 11 & 22(1) & \mathrm{C} 1 & \mathrm{O} 1 & \mathrm{C} 4 & \mathrm{C} 3 & 0.9(10) \\ \mathrm{C} 1 & \mathrm{C} 2 & \mathrm{C} 3 & \mathrm{C} 4 & 0(1) & \mathrm{C} 2 & \mathrm{C} 1 & \mathrm{O} 1 & \mathrm{C} 4 & -0.9(9) \\ \mathrm{C} 5 & \mathrm{~N} 1 & \mathrm{C} 8 & \mathrm{C} 7 & -11(1) & \mathrm{C} 5 & \mathrm{C} 6 & \mathrm{C} 7 & \mathrm{C} 8 & -11(1) \\ \mathrm{C} 6 & \mathrm{C} 5 & \mathrm{~N} 1 & \mathrm{C} 8 & 5(1) & \mathrm{C} 6 & \mathrm{C} 5 & \mathrm{~N} 1 & \mathrm{C} 9 & 178(1) \\ \mathrm{C} 7 & \mathrm{C} 8 & \mathrm{~N} 1 & \mathrm{C} 9 & 175(1) & \mathrm{C} 10 & \mathrm{~N} 2 & \mathrm{C} 13 & \mathrm{C} 12 & -16(1) \\ \mathrm{C} 10 & \mathrm{C} 11 & \mathrm{C} 12 & \mathrm{C} 13 & -21(1) & \mathrm{C} 11 & \mathrm{C} 10 & \mathrm{~N} 2 & \mathrm{C} 13 & 4(1) \\ \mathrm{C} 11 & \mathrm{C} 10 & \mathrm{~N} 2 & \mathrm{C} 14 & 176.2(10) & \mathrm{C} 12 & \mathrm{C} 13 & \mathrm{~N} 2 & \mathrm{C} 14 & 171.1(10)\end{array}$


Table 8. Non-bonded Contacts out to $3.60 \AA$

$\begin{array}{llllllll}\text { atom } & \text { atom } & \text { distance } & \text { ADC } & \text { atom } & \text { atom } & \text { distance } & \text { ADC } \\ \text { I1 } & \text { O3 } & 2.888(7) & 1 & \text { I2 } & \text { O2 } & 2.901(7) & 1 \\ \text { O1 } & \mathrm{C} 14 & 3.56(1) & 66501 & \mathrm{O} 1 & \mathrm{C} 7 & 3.59(2) & 65501 \\ \mathrm{O} 1 & \mathrm{C} 6 & 3.60(1) & 65501 & \mathrm{O} 2 & \mathrm{C} 4 & 3.15(1) & 66402 \\ \mathrm{O} 2 & \mathrm{C} 11 & 3.60(2) & 55402 & \mathrm{O} 3 & \mathrm{C} 1 & 3.15(1) & 65502 \\ \mathrm{O} 3 & \mathrm{C} 11 & 3.51(2) & 2 & \mathrm{O} 3 & \mathrm{C} 12 & 3.56(1) & 2 \\ \mathrm{C} 4 & \mathrm{C} 7 & 3.56(2) & 65501 & & & & \end{array}$


X-ray crystallographic data for compound ( \pm -26

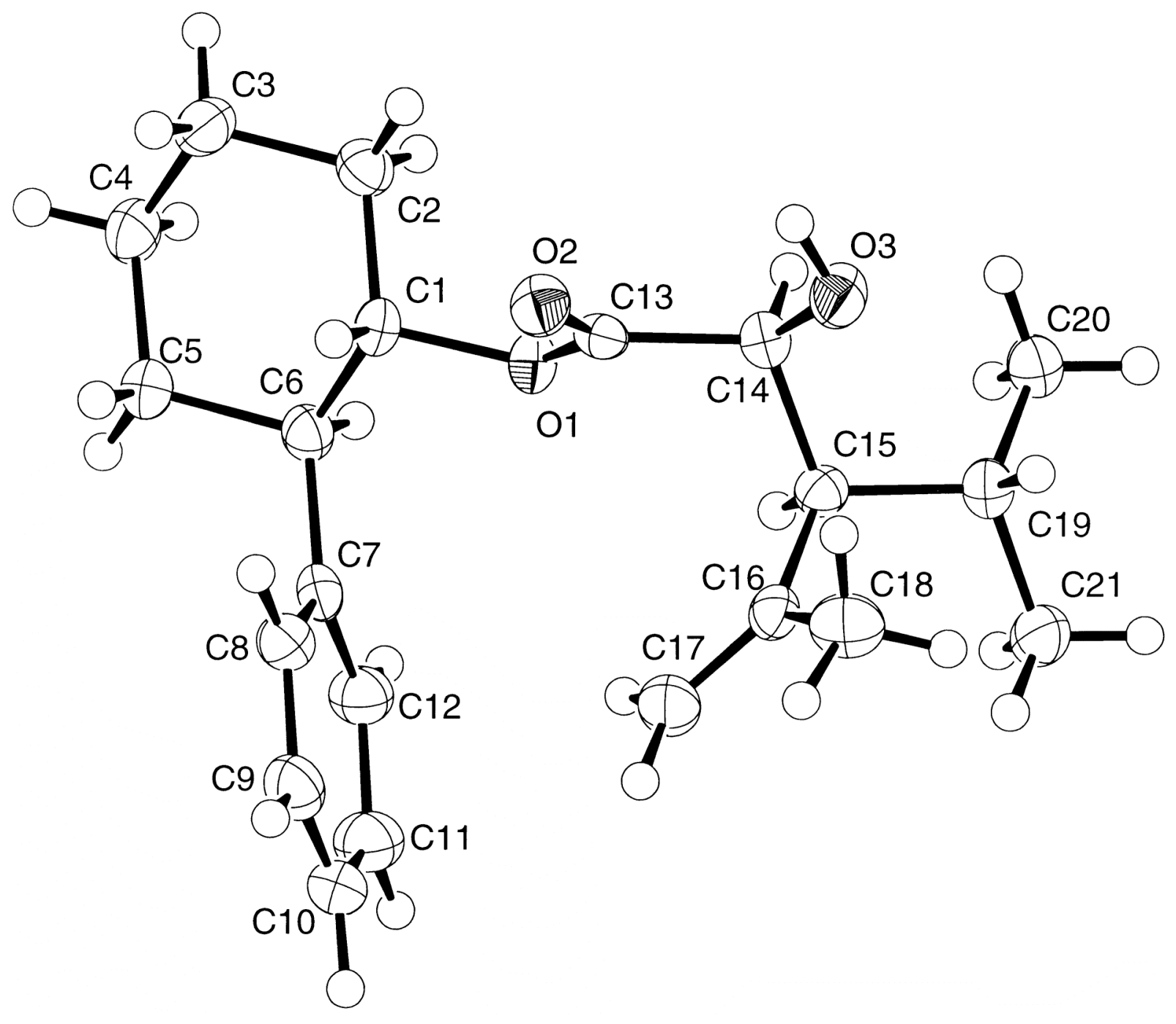




\section{A. Crystal Data}

Empirical Formula Formula Weight Crystal Color, Habit Crystal Dimensions Crystal System

Lattice Type

lattice Parameters

Space (iroup)

$Z$ value

D) ralc

$\mathrm{F}_{\text {(000) }}$

$\mu($ Mokn $)$

Diffractometer

Radiation

Detector Position

Exposure 'Time

Scan Type

$2 \theta_{\max }$

o. of Reflections Measured

('orrections

$49.5^{\circ}$

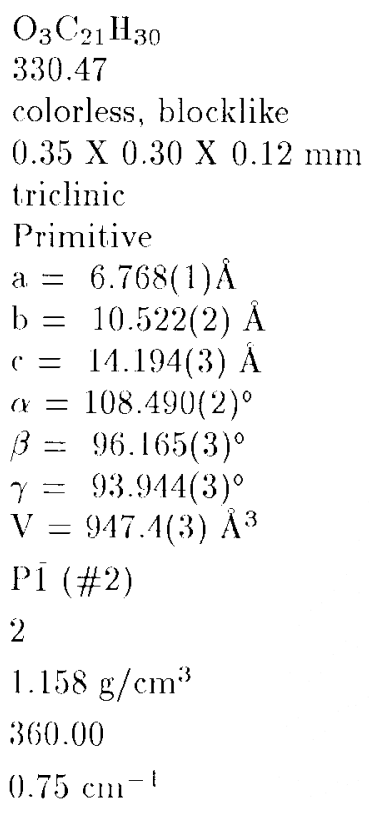

B. Intensity Mcasurements

\section{Bruker SMART CCD}

$\operatorname{Mok} a x(\lambda=0.71069 \hat{A})$

graphite monochromated

$60.00 \mathrm{~mm}$

10.0 seconds per frame.

$\omega(0.3$ degrees per frame)

Total: 5357

Unique: $2064\left(\mathrm{R}_{\text {int }}=0.030\right)$

Lorentz-polarization

Absorption ('Tmax $=1.00 \mathrm{~T}$ min $=0.80)$

C. Structure Solution and Refinement

Structure Solution

Refinement.

Function Minimized

Least Squares Weights

p-factor

Anomalous Dispersion
Direct Methods (SIR97)

Full-matrix least-squares

$\Sigma w\left(\left|F_{o}\right|-|F c|\right)^{2}$

$w=\frac{1}{\sigma^{2}(F o)}=\left[\sigma_{c}^{2}(F o)+\frac{p^{2}}{4} F o^{2}\right]^{-1}$

0.0300

All non-hydrogen atoms 
No. Observations $(\mathrm{I}>3.00 \sigma(\mathrm{I}))$

2090

No. Variables

217

Reflection/Parameter Ratio

9.63

Residuals: R; Rw; Rall

$0.053 ; 0.076 ; 0.080$

Goodness of Fit Indicator

1.48

Max Shift/Error in Final Cycle

0.00

Maximum peak in Final Diff. Map

$0.29 \epsilon^{-} / \AA^{3}$

Minimum peak in Final Diff. Map

$-0.19 e^{-/ \AA^{3}}$ 
Table 1. Atomic coordinates and $\mathrm{B}_{i s o} / \mathrm{B}_{e q}$

\begin{tabular}{|c|c|c|c|c|}
\hline atom & $\mathrm{x}$ & $\mathrm{y}$ & z & $\mathrm{B}_{e q}$ \\
\hline O1 & $0.3537(2)$ & $0.1594(2)$ & $0.1960(1)$ & $2.13(4)$ \\
\hline $\mathrm{O}_{2}$ & $0.0613(3)$ & $0.0397(2)$ & $0.1148(1)$ & $2.51(4)$ \\
\hline $\mathrm{O} 3$ & $-0.0161(3)$ & $0.2279(2)$ & $0.0238(1)$ & $2.78(4)$ \\
\hline $\mathrm{C} 1$ & $0.4235(4)$ & $0.0439(2)$ & $0.2213(2)$ & $2.01(6)$ \\
\hline $\mathrm{C}_{2}$ & $0.5419(4)$ & $-0.0304(3)$ & $0.1399(2)$ & $2.39(6)$ \\
\hline $\mathrm{C} 3$ & $0.6368(4)$ & $-0.1446(3)$ & $0.1663(2)$ & $2.79(7)$ \\
\hline C.4 & $0.7591(4)$ & $-0.0932(3)$ & $0.2684(2)$ & $2.89(7)$ \\
\hline $\mathrm{C} 5$ & $0.6362(4)$ & $-0.0206(3)$ & $0.3480(2)$ & $2.47(6)$ \\
\hline$(16)$ & $0.5474(4)$ & $0.0977(3)$ & $0.3241(2)$ & $2.14(6)$ \\
\hline$(7$ & $0.4349(4)$ & $0.1744(3)$ & $0.4071(2)$ & $2.08(6)$ \\
\hline$(8)$ & $0.2484(4)$ & $0.1241(3)$ & $0.4199(2)$ & $2.50(6)$ \\
\hline $\mathrm{C} 9$ & $0.1501(4)$ & $0.1935(3)$ & $0.4971(2)$ & $2.94(7)$ \\
\hline C:10 & $0.2362(5)$ & $0.3158(3)$ & $0.5641(2)$ & $3.12(7)$ \\
\hline$(\because 11$ & $0.4231(5)$ & $(0.3661(3)$ & $0.5539(2)$ & $3.27(7)$ \\
\hline$(12$ & $0.5206(4)$ & $0.2964(3)$ & $0.4757(2)$ & $2.73(7)$ \\
\hline$(11: 3$ & $0.1796(4)$ & $0.1399(.3)$ & $0.1368(2)$ & $1.98(6)$ \\
\hline$(114$ & $0.1561(4)$ & $0.25 .52(3)$ & $0.0953(2)$ & $2.14(6)$ \\
\hline$(: 15)$ & $0.1509(4)$ & $0.3909(2)$ & $0.1775(2)$ & $1.97(6)$ \\
\hline$(! 16$ & $-0.0081(4)$ & ().38.51(2) & $0.2436(2)$ & $2.0 \times(6)$ \\
\hline$(\because 17$ & $0.0421(4)$ & $0.38 .32(3)$ & $0.3367(2)$ & $2.97(7)$ \\
\hline C18 & $-0.2214(4)$ & $0.3816(3)$ & $0.2028(2)$ & $3.11(7)$ \\
\hline C19 & $0.1385(1)$ & $0.50(055(3)$ & $0.1332(2)$ & $2.17(6)$ \\
\hline $\mathrm{C} 20$ & $0.2968(4)$ & $0.5107(3)$ & $0.0664(2)$ & $2.5(0)(6)$ \\
\hline$C 21$ & $0.1528(4)$ & $0.6424(3)$ & $0.2170(2)$ & $2.922(7)$ \\
\hline III & 0.3117 & -0.0145 & 0.2233 & 2.4116 \\
\hline 112 & 0.45 .52 & -0.0670 & 0.0783 & 2.8638 \\
\hline 113 & 0.6445 & 0.0311 & 0.1330 & 2.8638 \\
\hline III & 0.5839 & -0.2111 & 0.1658 & 3.3362 \\
\hline 115 & 0.7207 & -0.1830 & 0.1177 & 3.3362 \\
\hline 116 & 0.8078 & -0.1673 & 0.2846 & 3.46 .55 \\
\hline 117 & 0.8686 & -0.0326 & 0.2671 & 3.46 .55 \\
\hline 118 & 0.5305 & -0.0823 & 0.3516 & 2.9699 \\
\hline 119 & 0.7195 & 0.0128 & 0.4109 & 2.9699 \\
\hline II10 & 0.6554 & 0.1579 & 0.3204 & 2.5602 \\
\hline H11 & 0.1872 & 0.0399 & 0.3742 & 3.0035 \\
\hline H12 & 0.0225 & 0.1569 & 0.5044 & 3.5291 \\
\hline H13 & 0.1674 & 0.3648 & 0.6167 & 3.7300 \\
\hline H14 & 0.4854 & 0.4491 & 0.6008 & 3.9222 \\
\hline H15 & 0.6486 & 0.3330 & 0.4690 & 3.2780 \\
\hline
\end{tabular}


Table 1. Atomic coordinates and $\mathrm{B}_{i s o} / \mathrm{B}_{e q}$ (continued)

\begin{tabular}{|c|c|c|c|c|}
\hline atom & $\mathrm{x}$ & y & $\mathrm{z}$ & $\mathrm{B}_{e q}$ \\
\hline II16 & 0.2688 & 0.2635 & 0.0622 & 2.5554 \\
\hline H17 & 0.2757 & 0.4090 & 0.2195 & 2.3539 \\
\hline H18 & 0.1786 & 0.3854 & 0.3618 & 3.5527 \\
\hline H19 & -0.0587 & 0.3797 & 0.3777 & 3.5527 \\
\hline II 20 & -0.2413 & 0.4636 & 0.1902 & 3.7286 \\
\hline 1121 & -0.2543 & 0.3082 & 0.1421 & 3.7286 \\
\hline $\mathrm{H} 22$ & -0.3046 & 0.3709 & 0.2502 & 3.7286 \\
\hline $\mathrm{H} 23$ & 0.0116 & 0.4929 & 0.0937 & 2.6045 \\
\hline 1124 & 0.287 .3 & 0.4265 & 0.0143 & 3.0020 \\
\hline HI25 & 0.2773 & 0.5802 & 0.0380 & 3.0020 \\
\hline$H 26$ & 0.425 .5 & 0.5284 & 0.1050 & 3.0020 \\
\hline 1127 & 0.2814 & 0.66066 & 0.2557 & 3.5110 \\
\hline $\mathrm{H} 28$ & 0.1324 & 0.7116 & 0.188 .5 & 3.5110 \\
\hline $1[29$ & 0.0535 & (0.6:393 & 0.2590 & 3.5110 \\
\hline II 30 & -0.0357 & 0.1442 & $-(0.0146$ & 3.8290 \\
\hline
\end{tabular}


Table 2. Anisotropic Displacement Parameters

\begin{tabular}{|c|c|c|c|c|c|c|}
\hline atom & $\mathrm{U}_{11}$ & $\mathrm{U}_{22}$ & $\mathrm{U}_{33}$ & $\mathrm{U}_{12}$ & $\mathrm{U}_{13}$ & $\mathrm{I}_{23}^{\top}$ \\
\hline O1 & $0.028(1)$ & $0.0245(10)$ & $0.031(1)$ & $0.0023(7)$ & $0.0019(9)$ & $0.01333(8)$ \\
\hline $\mathrm{O} 2$ & $0.034(1)$ & $0.026(1)$ & $0.032(1)$ & $-0.0024(8)$ & $-0.0003(10)$ & $0.0075(8)$ \\
\hline $\mathrm{O} 3$ & $0.041(1)$ & $0.029(1)$ & $0.031(1)$ & $0.0051(8)$ & $-0.0084(10)$ & $0.0071(9)$ \\
\hline $\mathrm{C} 1$ & $0.028(1)$ & $0.021(1)$ & $0.030(2)$ & $0.003(1)$ & $0.003(1)$ & $0.012(1)$ \\
\hline $\mathrm{C} 2$ & $0.032(2)$ & $0.032(2)$ & $0.026(2)$ & $0.003(1)$ & $0.002(1)$ & $0.010(1)$ \\
\hline $\mathrm{CB}$ & $0.040(2)$ & $0.028(2)$ & $0.040(2)$ & $0.009(1)$ & $0.015(2)$ & $0.011(1)$ \\
\hline $\mathrm{CA}$ & $0.038(2)$ & $0.040(2)$ & $0.040(2)$ & $0.017(1)$ & $0.010(2)$ & $0.020(1)$ \\
\hline C.5 & $0.035(2)$ & $0.031(1)$ & $0.032(2)$ & $0.009(1)$ & $0.005(1)$ & $0.014(1)$ \\
\hline $\mathrm{C} 6$ & $0.028(1)$ & $0.028(1)$ & $0.028(2)$ & $0.003(1)$ & $0.005(1)$ & $0.012(1)$ \\
\hline$(7)$ & $0.030(2)$ & $0.028(1)$ & $0.025(2)$ & $0.005(1)$ & $0.003(1)$ & $0.014(1)$ \\
\hline$(88$ & $0.036(2)$ & $0.031(2)$ & $0.029(2)$ & $0.003(1)$ & $0.006(1)$ & $0.011(1)$ \\
\hline$(9)$ & $0.035(2)$ & $0.045(2)$ & $0.039(2)$ & $0.009(1)$ & $0.013(2)$ & $0.0222(2)$ \\
\hline$(10$ & $0.055 .5(2)$ & $0.040(2)$ & $0.030(2)$ & $0.017(2)$ & $0.015(2)$ & $0.014(1)$ \\
\hline C:11 & $0.050(2)$ & $0.036(2)$ & $0.036(2)$ & $0.0002(1)$ & $0.010(2)$ & $0.00 \pi(1)$ \\
\hline$(12$ & $0.038(2)$ & $0.032(2)$ & $0.032(2)$ & $0.001(1)$ & $0.006(1)$ & $0.00 \times(1)$ \\
\hline$(13)$ & $0.029(1)$ & $0.028(1)$ & $0.019(1)$ & $0.006(1)$ & $0.006(1)$ & $0.0066(1)$ \\
\hline$(14$ & $0.0299(1)$ & $0.027(1)$ & $0.026(1)$ & $0.004(1)$ & $0.0033(1)$ & $0.011(1)$ \\
\hline$(15)$ & $0.025(1)$ & (0.024(1) & $0.024(1)$ & $0.003(1)$ & $0.001(1)$ & $0.006(1)$ \\
\hline$C: 16$ & $0.030(1)$ & $0.021(1)$ & $0.030(2)$ & $0.0003(1)$ & $0.007(1)$ & $0.010(1)$ \\
\hline$C 17$ & $0.038(2)$ & $0.044(2)$ & $0.039(2)$ & $0 .(013(1)$ & $0.016(2)$ & $0.01 ! 9(1)$ \\
\hline$(18$ & $0 .(030(2)$ & $0.046(2)$ & $0.043(2)$ & $0.003(1)$ & $0.013(2)$ & $0 .(113(1)$ \\
\hline C19 & $0.025(1)$ & $0.029(1)$ & $0.0332(2)$ & $0.004(1)$ & $0.004(1)$ & $(0.015(1)$ \\
\hline (:20) & $0.0334(2)$ & $0.032(2)$ & $0.0332(2)$ & $0.004(1)$ & $0.005(1)$ & $0.011(1)$ \\
\hline$(, 21$ & $0.045(2)$ & $0.030(2)$ & $0.043(2)$ & $0.008(1)$ & $0.019(2)$ & $(0.016(1)$ \\
\hline
\end{tabular}

The general temperature factor expression:

$$
\exp \left(-2 \pi^{2}\left(a^{* 2} U_{11} h^{2}+b^{* 2} U_{22} k^{2}+c^{* 2} U_{33} l^{2}+2 a^{*} b^{*} U_{12} h k+2 a^{*} c^{*} U_{13} h l+2 b^{*} c^{*} U_{23} h l\right)\right)
$$


Table 3. Bond Lengths $(\AA)$

$\begin{array}{llllll}\text { atom } & \text { atom } & \text { distance } & \text { atom } & \text { atom } & \text { distance } \\ \mathrm{O} 1 & \mathrm{C} 1 & 1.466(3) & \mathrm{O} 1 & \mathrm{C} 13 & 1.334(3) \\ \mathrm{O} 2 & \mathrm{C} 13 & 1.213(3) & \mathrm{O} 3 & \mathrm{C} 14 & 1.410(3) \\ \mathrm{C} 1 & \mathrm{C} 2 & 1.517(4) & \mathrm{C} 1 & \mathrm{C} 6 & 1.517(4) \\ \mathrm{C} 2 & \mathrm{C} 3 & 1.529(4) & \mathrm{C} 3 & \mathrm{C} 4 & 1.505(4) \\ \mathrm{C} 4 & \mathrm{C} 5 & 1.515(4) & \mathrm{C} 5 & \mathrm{C} 6 & 1.534(4) \\ \mathrm{C} 6 & \mathrm{C} 6 & 1.515(4) & \mathrm{C} 7 & \mathrm{C} 8 & 1.387(4) \\ \mathrm{C} 7 & \mathrm{C} 12 & 1.385(4) & \mathrm{C} 8 & \mathrm{C} 9 & 1.376(4) \\ \mathrm{C} 9 & \mathrm{C} 10 & 1.378(4) & \mathrm{C} 10 & \mathrm{C} 11 & 1.377(4) \\ \mathrm{C} 11 & \mathrm{C} 12 & 1.383(4) & \mathrm{C} 13 & \mathrm{C} 14 & 1.518(4) \\ \mathrm{C} 14 & \mathrm{C} 15 & 1.536(4) & \mathrm{C} 15 & \mathrm{C} 16 & 1.512(4) \\ \mathrm{C} 15 & \mathrm{C} 19 & 1.539(4) & \mathrm{C} 16 & \mathrm{C} 17 & 1.334(4) \\ \mathrm{C} 16 & \mathrm{C} 18 & 1.490(4) & \mathrm{C} 19 & \mathrm{C} 20 & 1.513(4) \\ \mathrm{C} 19 & \mathrm{C} 21 & 1.532(4) & & & \end{array}$

Table 4. Bond Lengths $(\AA)$

$\begin{array}{llllll}\text { atom } & \text { atom } & \text { distance } & \text { atom } & \text { atom } & \text { distance } \\ \mathrm{O} 3 & \mathrm{H} 30 & 0.87 & \mathrm{C} 1 & \mathrm{H} 1 & 0.95 \\ \mathrm{C} 2 & \mathrm{H} 2 & 0.95 & \mathrm{C} 2 & \mathrm{H} 3 & 0.95 \\ \mathrm{C} 3 & \mathrm{H} 4 & 0.95 & \mathrm{C} 3 & \mathrm{H} 5 & 0.95 \\ \mathrm{C} 4 & \mathrm{H} 6 & 0.95 & \mathrm{C} 4 & \mathrm{H} 7 & 0.95 \\ \mathrm{C} 5 & \mathrm{H} 8 & 0.95 & \mathrm{C} 5 & \mathrm{H} 9 & 0.95 \\ \mathrm{C} 6 & \mathrm{H} 10 & 0.95 & \mathrm{C} 8 & \mathrm{H} 11 & 0.95 \\ \mathrm{C} 9 & \mathrm{H} 12 & 0.95 & \mathrm{C} 10 & \mathrm{H} 13 & 0.95 \\ \mathrm{C} 11 & \mathrm{H} 14 & 0.95 & \mathrm{C} 12 & \mathrm{H} 15 & 0.95 \\ \mathrm{C} 14 & \mathrm{H} 16 & 0.95 & \mathrm{C} 15 & \mathrm{H} 17 & 0.95 \\ \mathrm{C} 17 & \mathrm{H} 18 & 0.95 & \mathrm{C} 17 & \mathrm{H} 17 & 0.95 \\ \mathrm{C} 18 & \mathrm{H} 20 & 0.95 & \mathrm{C} 18 & \mathrm{H} 21 & 0.95 \\ \mathrm{C} 18 & \mathrm{H} 22 & 0.95 & \mathrm{C} 19 & \mathrm{H} 23 & 0.95 \\ \mathrm{C} / 20 & \mathrm{H} 24 & 0.95 & \mathrm{C} 20 & \mathrm{H} 25 & 0.95 \\ \mathrm{C} 20 & \mathrm{H} 26 & 0.95 & (21 & \mathrm{H} 27 & 0.95 \\ \mathrm{C} 21 & \mathrm{H} 28 & 0.95 & \mathrm{C} 21 & \mathrm{H} 29 & 0.95\end{array}$


Table 5. Bond Angles( $\left.{ }^{\circ}\right)$

\begin{tabular}{|c|c|c|c|c|c|c|c|}
\hline atom & atom & atom & angle & atom & atom & atom & angle \\
\hline $\mathrm{C} 1$ & $\mathrm{O} 1$ & $\mathrm{C} 13$ & $117.8(2)$ & O1 & $\mathrm{C} 1$ & $(\because 2$ & $107.8(2)$ \\
\hline $\mathrm{O} 1$ & $\mathrm{C} 1$ & $\mathrm{C} 6$ & $107.7(2)$ & $\mathrm{C} 2$ & C1 & $\mathrm{C} 6$ & $112.8(2)$ \\
\hline $\mathrm{C} 1$ & $\mathrm{C} 2$ & C3 & $110.7(2)$ & $\mathrm{C} 2$ & $\mathrm{C} 3$ & $(4$ & $111.0(2)$ \\
\hline $\mathrm{C} 3$ & $C 4$ & C5 & $111.5(2)$ & C. 4 & $C 5$ & $\mathrm{C} 6$ & $111.4(2)$ \\
\hline C1 & $(6)$ & $C 5$ & $108.4(2)$ & $\mathrm{C} 1$ & C6 & $\mathrm{Cit}$ & $114.4(2)$ \\
\hline$(5)$ & $\mathrm{C} 6$ & $C 7$ & $110.9(2)$ & $\mathrm{C} 6$ & $C 7$ & C8 & $122.1(2)$ \\
\hline$(6$ & 67 & 012 & $120.1(2)$ & $(8$ & $(7$ & $(12$ & $117.7(3)$ \\
\hline$(7$ & $C 8$ & $(9$ & $121.4(3)$ & $\mathrm{C} 8$ & $\mathrm{C} 9$ & $(: 10$ & $120.2(3)$ \\
\hline $\mathrm{CO}$ & $\mathrm{C} 10$ & C11 & $119.2(3)$ & $\mathrm{C} 10$ & (:11 & $0: 12$ & $120.3(3)$ \\
\hline$C 7$ & C12 & C:11 & $121.1(3)$ & 01 & (:13 & $\mathrm{O} 2$ & $124.4(2)$ \\
\hline 01 & C.13 & C14 & $110.9(2)$ & $\mathrm{O} 2$ & $\mathrm{C} 13$ & $\mathrm{C} 14$ & $124.5(2)$ \\
\hline O.3 & $\mathrm{C} 14$ & C:13 & $111.2(2)$ & O3 & (:14 & C.15 & $109.8(2)$ \\
\hline (:13 & C:14 & ('15 & $112.8(2)$ & (\$14 & $(15$ & (:16 & $112.4(2)$ \\
\hline C:14 & $(15$ & C:19 & $111.3(2)$ & C.16 & $(: 15)$ & $\mathrm{C} 19$ & $113.3(2)$ \\
\hline$(15$ & $(16$ & $(17$ & $120.5(2)$ & C15 & C16 & C.18 & $118.7(2)$ \\
\hline C17 & $(16$ & C.18 & $120.8(3)$ & $(15$ & $(119$ & $(20)$ & $113.0(2)$ \\
\hline (15) & (11) & $(21$ & $110.5(2)$ & $\mathrm{C} 20$ & (:19) & $(21$ & $109.7(2)$ \\
\hline
\end{tabular}


Table 6. Bond Angles $\left({ }^{\circ}\right)$

\begin{tabular}{|c|c|c|c|c|c|c|c|}
\hline atom & atom & atom & angle & atom & atom & atom & angle \\
\hline C14 & $\mathrm{O} 3$ & $\mathrm{H} 30$ & 112.7 & O1 & $\mathrm{C} 1$ & $\mathrm{H} 1$ & 109.5 \\
\hline $\mathrm{C} 2$ & $\mathrm{C} 1$ & $\mathrm{H} 1$ & 109.5 & C6 6 & C.1 & H1 & 109.5 \\
\hline C1 & $\mathrm{C} 2$ & $\mathrm{H} 2$ & 109.2 & $\mathrm{C} 1$ & $\mathrm{C} 2$ & Н3 & 109.2 \\
\hline C3 & $\mathrm{C} 2$ & $\mathrm{II} 2$ & 109.1 & $\mathrm{C} 3$ & $(2$ & H3 & 109.1 \\
\hline $\mathrm{H} 2$ & $(2$ & 113 & 109.4 & $\mathrm{C} 2$ & $\mathrm{C} 3$ & II4 & 109.0 \\
\hline $\mathrm{C} 2$ & ( 3 & H5 & 109.1 & $C 4$ & $\mathrm{C} 3$ & $\mathrm{H} 4$ & 109.1 \\
\hline $\mathrm{C} 4$ & $(3)$ & II 5 & 109.1 & $\mathrm{H} 4$ & (3) & $\mathrm{H} 5$ & 109.5 \\
\hline$(3$ & $(4$ & 116 & 109.0 & C3 & C.4 & 117 & 108.9 \\
\hline$(5)$ & $C 4$ & 116 & 109.0 & $\mathrm{C} 5$ & $(14$ & $\mathrm{HI} 7$ & 109.0 \\
\hline 116 & C. & $\mathrm{H} 7$ & 109.5 & $\mathrm{CA}$ & $(5)$ & $\mathrm{H} 8$ & 109.0 \\
\hline$(4$ & $C 5$ & 119 & 108.9 & $(6$ & $\mathrm{C} 5$ & $\mathrm{H} 8$ & 109.0 \\
\hline C6 & $(5)$ & $\mathrm{H} 9$ & 109.0 & $\mathrm{H} 8$ & $(5)$ & 119 & 109.5 \\
\hline C1 & $c 6$ & II10 & 107.6 & (5) & $c 6$ & H10 & 107.7 \\
\hline$C 7$ & $C 6$ & H10 & 107.6 & $(7$ & $C 8$ & H 11 & 119.3 \\
\hline $\mathrm{C} 9$ & $C 8$ & H11 & 119.3 & $\mathrm{C} 8$ & C) & $\mathrm{H} 12$ & 119.9 \\
\hline C10 & $(9$ & 1112 & 119.9 & $C 9$ & $(10)$ & H1 13 & 120.4 \\
\hline C11 & $C 10$ & $111: 3$ & 120.4 & $\mathrm{CIO}$ & $\mathrm{C} 11$ & 1114 & 119.9 \\
\hline$(\because 12$ & C.11 & IIIA & 119.8 & $(7$ & $(12$ & III5 & 119.5 \\
\hline (11 & $(12$ & 1115 & 119.4 & 03 & $(14$ & II 16 & 107.6 \\
\hline ('1:3 & $(14$ & 1116 & 107.5 & (1) & C.1. & 1116 & 107.6 \\
\hline ('14 & (15) & 1117 & 106.4 & 016 & $(15)$ & $\mathrm{H} 17$ & 1066.4 \\
\hline$(19)$ & $(15)$ & 1117 & 106.4 & C16 & $(: 17$ & $\mathrm{H} 18$ & 120.0 \\
\hline$(: 16$ & $(: 17$ & 1119 & 120.0 & П118 & $(: 17$ & 1119 & 120.0 \\
\hline$(\because 16$ & $(18$ & 1120 & 109.5 & C.16 & 018 & $\mathrm{H} 2 \mathrm{l}$ & 109.5 \\
\hline C16 & C18 & 1122 & 109.4 & 1120 & 018 & II2 1 & 109.5 \\
\hline $\mathrm{H} 20$ & $(18$ & $\mathrm{H} 22$ & 109.5 & $\mathrm{H} 21$ & $(18$ & $\mathrm{H} 22$ & 109.5 \\
\hline$(: 15)$ & $(19)$ & 1123 & 107.8 & (120) & $(19$ & 1123 & 107.8 \\
\hline (.21 & $(: 19$ & $\mathrm{H} 23$ & 107.8 & C19 & $(20$ & 1124 & 109.5 \\
\hline C:19 & $(20$ & 1125 & 109.5 & C19 & $(20$ & 1126 & 109.5 \\
\hline 1124 & $(20)$ & 1125 & 109.5 & II 24 & $\mathrm{C} 20$ & II 26 & 109.5 \\
\hline 1125 & $(20$ & 1126 & 109.4 & C.19 & C21 & $\mathrm{H} 27$ & 109.5 \\
\hline$(: 19)$ & $(\because 21$ & 1128 & 109.5 & C.19 & $\mathrm{C} 21$ & $\mathrm{H} 29$ & 109.5 \\
\hline $\mathrm{H} 2 \mathrm{~T}$ & $\mathrm{C} 21$ & II 28 & 109.5 & $\mathrm{H} 27$ & $\mathrm{C} 21$ & $\mathrm{H} 29$ & 109.4 \\
\hline 1128 & $\mathrm{C} \cdot 21$ & $\mathrm{H} 29$ & 109.5 & & & & \\
\hline
\end{tabular}


Table 7. Torsion Angles $\left(^{\circ}\right)$

\begin{tabular}{|c|c|c|c|c|c|c|c|c|c|}
\hline atom & atom & atom & a.tom & angle & atom & atom & atom & atom & angle \\
\hline $\mathrm{O} 1$ & () 1 & $\mathrm{C} 2$ & C.3 & $174.6(2)$ & O1 & $\mathrm{C} 1$ & C6 & C5 & $-175.4(2)$ \\
\hline $\mathrm{O} 1$ & $\mathrm{C} 1$ & $\mathrm{Co}$ & $\mathrm{C} 7$ & $60.3(3)$ & O1 & C13 & C:14 & O3 & $173.1(2)$ \\
\hline $\mathrm{O} 1$ & C 13 & C 14 & C 15 & $-63.0(3)$ & $\mathrm{O} 2$ & C13 & $\mathrm{Ol}$ & $\mathrm{C} 1$ & $11.6(4)$ \\
\hline $\mathrm{O} 2$ & $C 13$ & $\mathrm{C} 14$ & O3 & $-3.0(4)$ & $\mathrm{O}_{2}$ & C 13 & C14 & C 15 & $120.8(3)$ \\
\hline O 3 & C 14 & $(15)$ & C 16 & $70.0(3)$ & $\mathrm{O} 3$ & $(14$ & $C 15$ & C19 & $-58.4(3)$ \\
\hline $\mathrm{C} 1$ & 01 & (:13 & $(114$ & $-164.6(2)$ & $\mathrm{C} 1$ & $\mathrm{C} 2$ & $\mathrm{C} 3$ & C.4 & $-53.9(3)$ \\
\hline C 1 & $\mathrm{C} 6$ & C.5 & $(4$ & $56.9(3)$ & $\mathrm{C} 1$ & $C 6$ & $C 7$ & C8 & $48.2(3)$ \\
\hline$(' 1$ & $\mathrm{C} 6$ & $C 7$ & $C 12$ & $-134.2(2)$ & $\mathrm{C} 2$ & C1 & $\mathrm{O} 1$ & C:13 & $87.8(3)$ \\
\hline$(\because 2$ & $(1$ & $(6)$ & $(5)$ & $-56.6(3)$ & $(2$ & $(\vdots 1$ & $(6$ & $C 7$ & $179.1(2)$ \\
\hline$(\because 2$ & $(3)$ & $C 4$ & $(5)$ & $5.5 .5(3)$ & $(3)$ & $(2$ & $(1$ & $\mathrm{C} 6$ & $5.5 .8(3)$ \\
\hline C 3 & $(4$ & $(5)$ & $C 6$ & $-57.8(3)$ & $(, 4$ & $(5)$ & $(6)$ & $C 7$ & $-176.7(2)$ \\
\hline $\mathrm{C5}$ & $c 6$ & $C 7$ & 68 & $-74.8(3)$ & $(5)$ & $(66$ & $C 7$ & $(12$ & $102.8(3)$ \\
\hline $\mathrm{C} 6$ & $\mathrm{C} 1$ & 01 & $\mathrm{C} 13$ & $-150.2(2)$ & $C 6$ & $\mathrm{C} 7$ & $\mathrm{C} 8$ & $(9)$ & $178.4(2)$ \\
\hline$(6)$ & $C 7$ & $(12$ & C 11 & $-177.9(3)$ & $C 7$ & $\mathrm{C} 8$ & C9 & $(: 10$ & $-0.0(4)$ \\
\hline$(7)$ & $(112$ & $(: 11$ & $(: 10$ & $-1.0(4)$ & C8 & $C 7$ & 012 & ( 11 & $-0.3(4)$ \\
\hline$(8)$ & ( 9 & $(: 10$ & C:11 & $-1.3(4)$ & C9 & $\mathrm{C} 8$ & C.7 & $(112$ & $0.8(4)$ \\
\hline$(9$ & $(10$ & $(\div 11$ & $(: 12$ & $1.8(4)$ & $0: 13$ & $C: 14$ & $C: 15$ & $(: 16$ & $-54.7(3)$ \\
\hline$(13)$ & $(\mathrm{CH}$ & $(115)$ & ( :19 & $170.9(2)$ & C 11 & $(: 15)$ & $(: 16$ & $(17$ & $105.7(3)$ \\
\hline$(14$ & $(15)$ & $C: 16$ & $(: 18$ & $-74.4(3)$ & (:14 & $(: 15$ & $(: 19$ & $(120)$ & $-51.5(3)$ \\
\hline$(\div 14$ & $(15$ & $(19$ & $(: ; 21$ & $-174.8(2)$ & $(: 16$ & $C: 15$ & $(; 19$ & $(: 20)$ & $-179.4(2)$ \\
\hline$(\vdots 16$ & $(: 15$ & $(19$ & $(.21$ & $57.2(3)$ & C:17 & $(116$ & $(: 15$ & $(: 1 !)$ & $-126 . .9(3)$ \\
\hline ('18 & $(16)$ & $(15)$ & $(119$ & $53.0(3)$ & & & & & \\
\hline
\end{tabular}

Table 8. Non-bonded Contacts out to $3.60 \AA$

$\begin{array}{llllllll}\text { atom } & \text { atom } & \text { distance } & \text { ADC } & \text { atom } & \text { atom } & \text { distance } & \text { ADC } \\ \text { O1 } & \mathrm{C} 18 & 3.552(3) & 65501 & \mathrm{O} 2 & \mathrm{O} 3 & 2.851(3) & 2 \\ \mathrm{O} 2 & \mathrm{O} 2 & 3.098(4) & 2 & \mathrm{C} 5 & \mathrm{C} 9 & 3.528(4) & 65602 \\ \mathrm{C} 20 & \mathrm{C} 20 & 3.478(6) & 66502 & & & & \end{array}$




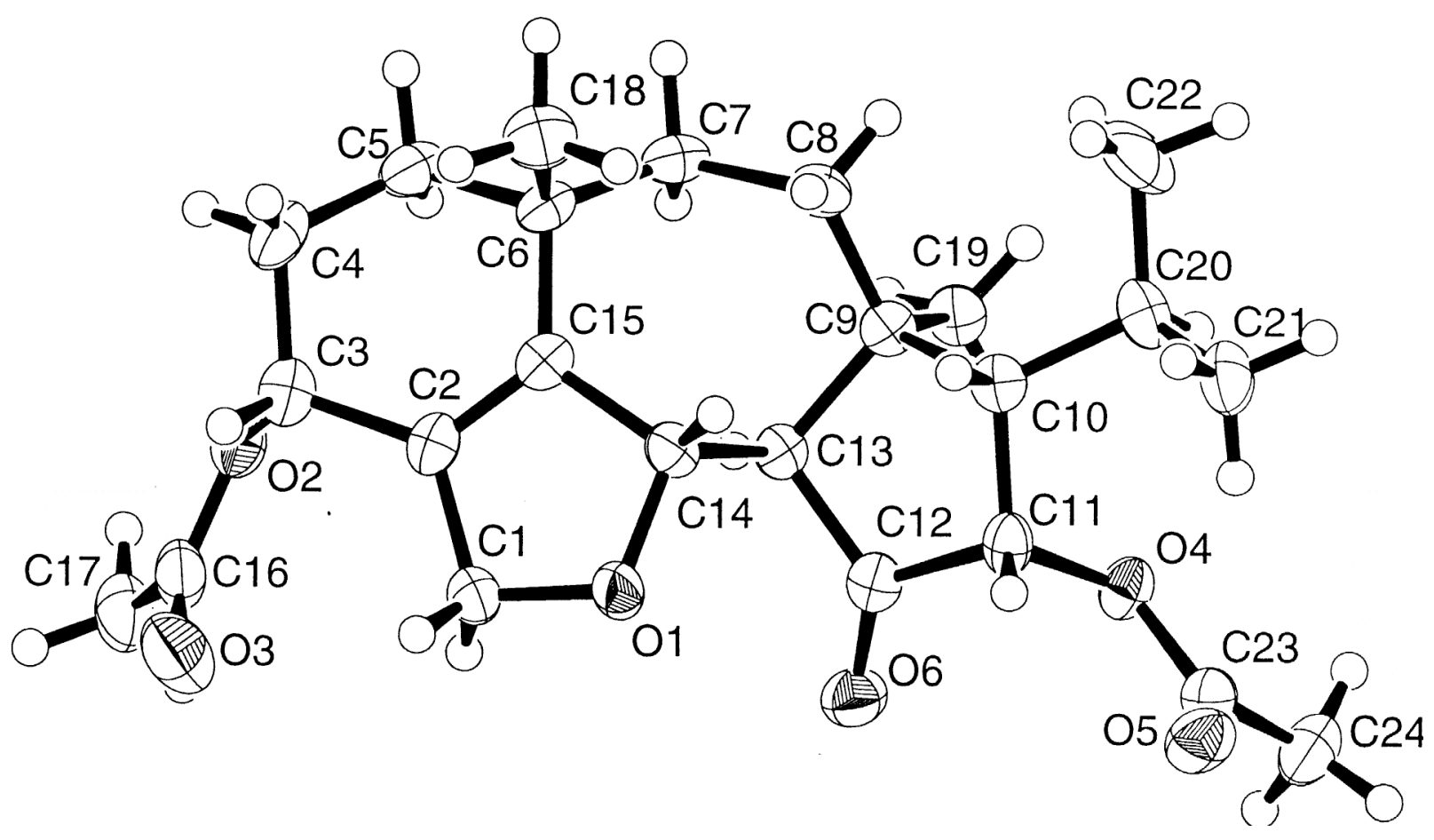


A. Crystal Data

\section{Empirical Formula \\ Formula Weight \\ Crystal Color, Habit \\ (rystal Dimensions \\ (rystal System \\ Lattice Type \\ Lattice Parameters}

Space Group

$\mathrm{Z}$ value

$\mathrm{D}_{\text {calc }}$

$\mathrm{F}_{000}$

$\mu(\operatorname{MoK} \alpha)$

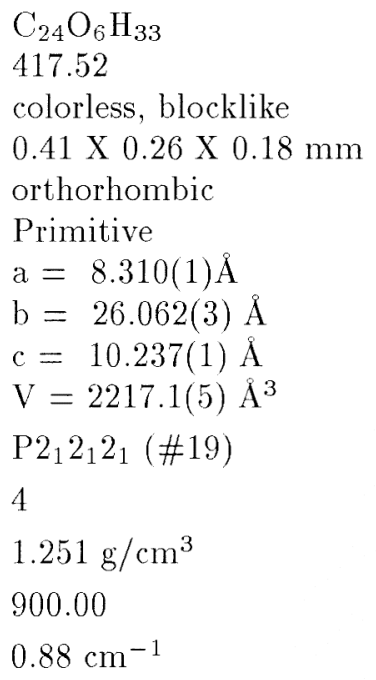

B. Intensity Measurements

Diffractometer

Radiation

Detector Position

Exposure Time

Scan Type

$2 \theta_{\max }$

No. of Reflections Measured

Corrections
Bruker APEX CCD

$\operatorname{MoK} \alpha(\lambda=0.71069 \AA)$ graphite monochromated

$60.00 \mathrm{~mm}$

10.0 seconds per frame.

$\omega$ (0.3 degrees per frame) $52.8^{\circ}$

Total: 12731

Unique: $3021\left(\mathrm{R}_{\text {int }}=0.042\right)$

Lorentz-polarization

Absorption $(\mathrm{T} \max =1.00 \mathrm{Tmin}=0.69)$

C. Structure Solution and Refinement

Structure Solution

Refinement

Function Minimized

Least Squares Weights

p-factor

Anomalous Dispersion

No. Observations (I>2.50\%(I))

No. Variables

Reflection/Parameter Ratio
Direct Methods (SIR97)

Full-matrix least-squares

$\Sigma w\left(\left|F_{O}\right|-|F c|\right)^{2}$

$w=\frac{1}{\sigma^{2}(F o)}=\left[\sigma_{c}^{2}(F o)+\frac{p^{2}}{4} F o^{2}\right]^{-1}$

0.0300

All non-hydrogen atoms

1904

271

7.03 
Residuals: R; Rw; Rall

Goodness of Fit Indicator

Max Shift/Error in Final Cycle

Maximum peak in Final Diff. Map

Minimum peak in Final Diff. Map
$0.042: 0.047 ; 0.068$

1.47

0.00

$0.15 e^{-} / \AA^{3}$

$-0.15 e^{-} / \AA^{3}$ 
Table 1. Atomic coordinates and $\mathrm{B}_{i s o} / \mathrm{B}_{e q}$

\begin{tabular}{|c|c|c|c|c|}
\hline atom & $\mathrm{x}$ & $\mathrm{y}$ & z & $\mathrm{B}_{e q}$ \\
\hline O 1 & $0.2895(2)$ & $0.22351(8)$ & $0.8466(2)$ & $2.80(5)$ \\
\hline $\mathrm{O} 2$ & $0.1957(2)$ & $0.07004(8)$ & $0.6674(2)$ & $2.68(5)$ \\
\hline O3 & $0.4376(3)$ & $0.06382(10)$ & $0.7642(3)$ & $4.58(7)$ \\
\hline $\mathrm{O} 4$ & $0.1998(2)$ & $0.39881(8)$ & $0.6615(2)$ & $2.44(5)$ \\
\hline $\mathrm{O} 5$ & $0.3546(3)$ & $0.44043(9)$ & $0.8083(3)$ & $3.45(6)$ \\
\hline 06 & $0.3687(3)$ & $0.30547(9)$ & $0.6439(2)$ & $3.11(5)$ \\
\hline $\mathrm{C} 1$ & $0.3212(3)$ & $0.1704(1)$ & $0.8224(3)$ & $2.52(7)$ \\
\hline $\mathrm{C} 2$ & $0.1594(4)$ & $0.1466(1)$ & $0.8015(3)$ & $2.17(7)$ \\
\hline C3 & $0.1275(4)$ & $0.0903(1)$ & $0.7899(3)$ & $2.72(7)$ \\
\hline $\mathrm{C} 4$ & $-0.0509(4)$ & $0.0786(1)$ & $0.7884(3)$ & $3.22(8)$ \\
\hline C5 & $-0.1496(4)$ & $0.1198(1)$ & $0.7200(3)$ & $2.97(8)$ \\
\hline C 6 & $-0.1310(4)$ & $0.1731(1)$ & $0.7833(3)$ & $2.35(7)$ \\
\hline$(T$ & $-0.2080(3)$ & $0.2134(1)$ & $0.6943(3)$ & $2.69(7)$ \\
\hline$(x)$ & $-0.1981(4)$ & $0.2698(1)$ & $0.7392(3)$ & $2.45(7)$ \\
\hline$(\because)$ & $-0.0511(3)$ & $0.2998(1)$ & $0.6905(3)$ & $2.14(6)$ \\
\hline$(10$ & $-0.0089(4)$ & $0.3470(1)$ & $0.7796(3)$ & $2.25(7)$ \\
\hline C 11 & $0.1698(4)$ & $0.3579(1)$ & $0.7543(3)$ & $2.34(7)$ \\
\hline $\mathrm{C} 12$ & $0.2369(4)$ & $0.3089(1)$ & $0.6927(3)$ & $2.27(7)$ \\
\hline $\mathrm{C} 13$ & $0.1073(3)$ & $0.2681(1)$ & $0.6956(3)$ & $2.02(6)$ \\
\hline C14 & $0.1229(3)$ & $0.2342(1)$ & $0.8187(3)$ & $2.15(6)$ \\
\hline $\mathrm{C} 15$ & $0.0469(4)$ & $0.1822(1)$ & $0.8013(3)$ & $2.10(6)$ \\
\hline C16 & $0.3511(4)$ & $0.0563(1)$ & $0.6713(4)$ & $3.13(8)$ \\
\hline $\mathrm{C} 17$ & $0.4043(5)$ & $0.0322(1)$ & $0.5464(4)$ & $3.77(9)$ \\
\hline C18 & $-0.2109(4)$ & $0.1734(1)$ & $0.9183(3)$ & $3.33(9)$ \\
\hline C19 & $-0.0770(4)$ & $0.3173(1)$ & $0.5486(3)$ & $2.63(7)$ \\
\hline $\mathrm{C} 20$ & $-0.1142(4)$ & $0.3954(1)$ & $0.7787(3)$ & $3.10(8)$ \\
\hline $\mathrm{C} 21$ & $-0.0559(4)$ & $0.4336(1)$ & $0.8797(4)$ & $3.30(8)$ \\
\hline $\mathrm{C} 22$ & $-0.2922(5)$ & $0.3853(2)$ & $0.8027(5)$ & $4.8(1)$ \\
\hline $\mathrm{C} 23$ & $0.3049(4)$ & $0.4361(1)$ & $0.6989(4)$ & $2.67(7)$ \\
\hline $\mathrm{C} 24$ & $0.3483(4)$ & $0.4698(1)$ & $0.5863(4)$ & $3.57(9)$ \\
\hline H1 & 0.3864 & 0.1664 & 0.7469 & 3.0188 \\
\hline $\mathrm{H} 2$ & 0.3735 & 0.1552 & 0.8954 & 3.0188 \\
\hline H3 & 0.1759 & 0.0731 & 0.8617 & 3.2568 \\
\hline $\mathrm{H} 4$ & -0.0671 & 0.0469 & 0.7445 & 3.8684 \\
\hline H5 & -0.0874 & 0.0756 & 0.8761 & 3.8684 \\
\hline $\mathrm{H} 6$ & -0.1157 & 0.1219 & 0.6315 & 3.5636 \\
\hline $\mathrm{H} 7$ & -0.2599 & 0.1103 & 0.7231 & 3.5636 \\
\hline H8 & -0.1570 & 0.2111 & 0.6114 & 3.2267 \\
\hline $\mathrm{H} 9$ & -0.3186 & 0.2049 & 0.6855 & 3.2267 \\
\hline
\end{tabular}


Table 1. Atomic coordinates and $\mathrm{B}_{i s o} / \mathrm{B}_{e q}$ (continued)

\begin{tabular}{|c|c|c|c|c|}
\hline atom & $\mathrm{x}$ & $\mathrm{y}$ & z & $\mathrm{B}_{e q}$ \\
\hline H10 & -0.2918 & 0.2869 & 0.7091 & 2.9388 \\
\hline H11 & -0.1962 & 0.2701 & 0.8320 & 2.9388 \\
\hline II12 & -0.0139 & 0.3345 & 0.8666 & 2.7020 \\
\hline 1113 & 0.2234 & 0.3649 & 0.8343 & 2.8009 \\
\hline 111.4 & 0.1153 & 0.2473 & 0.6196 & 2.4293 \\
\hline H15 & 0.0752 & 0.2511 & 0.8911 & 2.5859 \\
\hline H 16 & 0.3130 & 0.0253 & 0.4934 & 4.5182 \\
\hline $\mathrm{H} 17$ & 0.4593 & 0.0011 & 0.5647 & 4.5182 \\
\hline H18 & 0.4744 & 0.0550 & 0.5016 & 4.5182 \\
\hline H19 & -0.1931 & 0.2056 & 0.9592 & 3.9947 \\
\hline $\mathrm{H} 20$ & -0.3233 & 0.1678 & 0.9089 & 3.9947 \\
\hline $\mathrm{H} 21$ & -0.1659 & 0.1469 & 0.9706 & 3.9947 \\
\hline H22 & 0.0127 & 0.3371 & 0.5209 & 3.1498 \\
\hline $\mathrm{H} 23$ & -0.0877 & 0.2881 & 0.4938 & 3.1498 \\
\hline $\mathrm{H} 24$ & -0.1720 & 0.3375 & 0.5434 & 3.1498 \\
\hline H 25 & -0.1040 & 0.4110 & 0.6952 & 3.7183 \\
\hline H26 & -0.0673 & 0.4193 & 0.9645 & 3.9619 \\
\hline $\mathrm{H} 27$ & 0.0541 & 0.4414 & 0.8641 & 3.9619 \\
\hline H28 & -0.1180 & 0.4641 & 0.8738 & 3.9619 \\
\hline H29 & -0.3501 & 0.4166 & 0.7972 & 5.7178 \\
\hline H30 & -0.3315 & 0.3620 & 0.7387 & 5.7178 \\
\hline H31 & -0.3061 & 0.3709 & 0.8872 & 5.7178 \\
\hline H32 & 0.2543 & 0.4774 & 0.5371 & 4.2778 \\
\hline H33 & 0.4239 & 0.4526 & 0.5321 & 4.2778 \\
\hline H34 & 0.3942 & 0.5008 & 0.6179 & 4.2778 \\
\hline
\end{tabular}


Table 2. Anisotropic Displacement Parameters

\begin{tabular}{lcccccc} 
atom & $\mathrm{U}_{11}$ & $\mathrm{U}_{22}$ & $\mathrm{U}_{33}$ & $\mathrm{U}_{12}$ & \multicolumn{1}{c}{$\mathrm{U}_{13}$} & $\mathrm{U}_{23}$ \\
O1 & $0.031(1)$ & $0.026(1)$ & $0.048(1)$ & $0.0016(10)$ & $-0.014(1)$ & $-0.001(1)$ \\
O2 & $0.042(1)$ & $0.028(1)$ & $0.032(1)$ & $-0.001(1)$ & $0.002(1)$ & $-0.003(1)$ \\
O.3 & $0.055(2)$ & $0.053(2)$ & $0.065(2)$ & $0.017(1)$ & $-0.011(2)$ & $-0.011(1)$ \\
O.4 & $0.039(1)$ & $0.027(1)$ & $0.026(1)$ & $-0.005(1)$ & $-0.005(1)$ & $0.0005(9)$ \\
O.j & $0.042(1)$ & $0.043(1)$ & $0.045(2)$ & $-0.009(1)$ & $-0.008(1)$ & $-0.006(1)$ \\
O) & $0.033(1)$ & $0.040(1)$ & $0.045(1)$ & $-0.004(1)$ & $0.007(1)$ & $-0.004(1)$ \\
C1 & $0.031(2)$ & $0.028(2)$ & $0.036(2)$ & $0.002(1)$ & $-0.003(2)$ & $0.001(2)$ \\
C2 & $0.038(2)$ & $0.026(2)$ & $0.019(2)$ & $-0.003(1)$ & $0.004(1)$ & $0.001(1)$ \\
C3 & $0.045(2)$ & $0.030(2)$ & $0.028(2)$ & $-0.000(2)$ & $0.004(2)$ & $-0.001(1)$ \\
C4 & $0.051(2)$ & $0.028(2)$ & $0.043(2)$ & $-0.011(2)$ & $0.011(2)$ & $-0.007(2)$ \\
C5 & $0.033(2)$ & $0.038(2)$ & $0.042(2)$ & $-0.010(2)$ & $0.005(2)$ & $-0.008(2)$ \\
C6 & $0.029(2)$ & $0.031(2)$ & $0.030(2)$ & $-0.008(1)$ & $0.003(1)$ & $-0.004(1)$ \\
C7 & $0.026(1)$ & $0.042(2)$ & $0.035(2)$ & $-0.007(1)$ & $0.000(2)$ & $-0.006(2)$ \\
C8 & $0.028(2)$ & $0.037(2)$ & $0.028(2)$ & $0.002(2)$ & $-0.002(1)$ & $-0.002(1)$ \\
C9 & $0.031(2)$ & $0.030(2)$ & $0.021(2)$ & $0.000(1)$ & $-0.002(1)$ & $-0.004(1)$ \\
C10 & $0.033(2)$ & $0.030(2)$ & $0.023(2)$ & $0.001(1)$ & $-0.000(1)$ & $0.001(1)$ \\
C11 & $0.039(2)$ & $0.024(2)$ & $0.026(2)$ & $0.001(1)$ & $-0.007(1)$ & $0.001(1)$ \\
C12 & $0.034(2)$ & $0.029(2)$ & $0.023(2)$ & $-0.001(1)$ & $-0.003(2)$ & $0.000(1)$ \\
C13 & $0.028(2)$ & $0.027(2)$ & $0.022(2)$ & $0.000(1)$ & $0.002(1)$ & $-0.004(1)$ \\
C14 & $0.028(2)$ & $0.028(2)$ & $0.026(2)$ & $0.003(1)$ & $-0.002(1)$ & $-0.006(1)$ \\
C15 & $0.033(2)$ & $0.031(2)$ & $0.016(2)$ & $-0.004(1)$ & $0.002(1)$ & $-0.002(1)$ \\
C16 & $0.045(2)$ & $0.025(2)$ & $0.048(2)$ & $0.005(2)$ & $0.004(2)$ & $0.005(2)$ \\
C17 & $0.056(2)$ & $0.035(2)$ & $0.052(2)$ & $0.008(2)$ & $0.017(2)$ & $0.010(2)$ \\
C18 & $0.041(2)$ & $0.052(2)$ & $0.034(2)$ & $-0.003(2)$ & $0.010(2)$ & $-0.000(2)$ \\
C19 & $0.037(2)$ & $0.033(2)$ & $0.029(2)$ & $0.001(2)$ & $-0.005(2)$ & $-0.001(1)$ \\
C20 & $0.047(2)$ & $0.033(2)$ & $0.039(2)$ & $0.011(2)$ & $-0.002(2)$ & $0.006(2)$ \\
C21 & $0.052(2)$ & $0.029(2)$ & $0.045(2)$ & $0.008(2)$ & $0.011(2)$ & $0.000(2)$ \\
C22 & $0.044(2)$ & $0.056(3)$ & $0.081(3)$ & $0.024(2)$ & $-0.004(2)$ & $-0.023(2)$ \\
C23 & $0.031(2)$ & $0.029(2)$ & $0.042(2)$ & $-0.000(2)$ & $0.002(2)$ & $-0.002(2)$ \\
C24 & $0.051(2)$ & $0.033(2)$ & $0.051(2)$ & $-0.010(2)$ & $0.004(2)$ & $0.001(2)$ \\
& & & & &
\end{tabular}

The general temperature factor expression:

$$
\exp \left(-2 \pi^{2}\left(a^{* 2} U_{11} h^{2}+b^{* 2} U_{22} k^{2}+c^{* 2} U_{33} l^{2}+2 a^{*} b^{*} U_{12} h k+2 a^{*} c^{*} U_{13} h l+2 b^{*} c^{*} U_{23} k l\right)\right)
$$


Table 3. Bond Lengths $(\AA)$

$\begin{array}{llllll}\text { atom } & \text { atom } & \text { distance } & \text { atom } & \text { atom } & \text { distance } \\ \mathrm{O} 1 & \mathrm{C} 1 & 1.431(4) & \mathrm{O} 1 & \mathrm{C} 14 & 1.441(3) \\ \mathrm{O} 2 & \mathrm{C} 3 & 1.474(4) & \mathrm{O} 2 & \mathrm{C} 16 & 1.340(4) \\ \mathrm{O} 3 & \mathrm{C} 16 & 1.208(4) & \mathrm{O} 4 & \mathrm{C} 11 & 1.449(4) \\ \mathrm{O} 4 & \mathrm{C} 23 & 1.361(4) & \mathrm{O} 5 & \mathrm{C} 23 & 1.199(4) \\ \mathrm{O} 6 & \mathrm{C} 12 & 1.208(4) & \mathrm{C} 1 & \mathrm{C} 2 & 1.496(4) \\ \mathrm{C} 2 & \mathrm{C} 3 & 1.496(4) & \mathrm{C} 2 & \mathrm{C} 15 & 1.317(4) \\ \mathrm{C} 3 & \mathrm{C} 4 & 1.514(4) & \mathrm{C} 4 & \mathrm{C} 5 & 1.523(5) \\ \mathrm{C} 5 & \mathrm{C} 6 & 1.541(4) & \mathrm{C} 6 & \mathrm{C} 7 & 1.529(5) \\ \mathrm{C} 6 & \mathrm{C} 15 & 1.508(4) & \mathrm{C} 6 & \mathrm{C} 18 & 1.534(4) \\ \mathrm{C} 7 & \mathrm{C} 8 & 1.542(4) & \mathrm{C} 8 & \mathrm{C} 9 & 1.534(4) \\ \mathrm{C} 9 & \mathrm{C} 10 & 1.571(4) & \mathrm{C} 9 & \mathrm{C} 13 & 1.554(4) \\ (9 & \mathrm{C} 19 & 1.538(4) & \mathrm{C} 10 & \mathrm{C} 11 & 1.534(4) \\ (10 & \mathrm{C} 20 & 1.535(4) & \mathrm{C} 11 & \mathrm{C} 12 & 1.529(4) \\ (12 & \mathrm{C} 13 & 1.514(4) & \mathrm{C} 13 & \mathrm{C} 14 & 1.545(4) \\ (1.1 & \mathrm{C} 15 & 1.506(4) & \mathrm{C} 16 & \mathrm{C} 17 & 1.492(5) \\ (20 & \mathrm{C} 21 & 1.514(5) & \mathrm{C} 20 & \mathrm{C} 22 & 1.523(5) \\ \mathrm{C} 23 & \mathrm{C} 24 & 1.494(5) & & & \end{array}$


Table 4. Bond Lengths $(\AA)$

$\begin{array}{llllll}\text { atom } & \text { atom } & \text { distance } & \text { atom } & \text { atom } & \text { distance } \\ \mathrm{C} 1 & \mathrm{H} 1 & 0.95 & \mathrm{C} 1 & \mathrm{H} 2 & 0.95 \\ \mathrm{C} 3 & \mathrm{H} 3 & 0.95 & \mathrm{C} 4 & \mathrm{H} 4 & 0.95 \\ \mathrm{C} 4 & \mathrm{H} 5 & 0.95 & \mathrm{C} 5 & \mathrm{H} 6 & 0.95 \\ \mathrm{C} 5 & \mathrm{H} 7 & 0.95 & \mathrm{C} 7 & \mathrm{H} 8 & 0.95 \\ \mathrm{C} 7 & \mathrm{H} 9 & 0.95 & \mathrm{C} 8 & \mathrm{H} 10 & 0.95 \\ \mathrm{C} 8 & \mathrm{H} 11 & 0.95 & \mathrm{C} 10 & \mathrm{H} 12 & 0.95 \\ \mathrm{C} 11 & \mathrm{H} 13 & 0.95 & \mathrm{C} 13 & \mathrm{H} 14 & 0.95 \\ \mathrm{C} 14 & \mathrm{H} 15 & 0.95 & \mathrm{C} 17 & \mathrm{H} 16 & 0.95 \\ \mathrm{C} 17 & \mathrm{H} 17 & 0.95 & \mathrm{C} 17 & \mathrm{H} 18 & 0.95 \\ \mathrm{C} 18 & \mathrm{H} 19 & 0.95 & \mathrm{C} 18 & \mathrm{H} 20 & 0.95 \\ \mathrm{C} 18 & \mathrm{H} 21 & 0.95 & \mathrm{C} 19 & \mathrm{H} 22 & 0.95 \\ \mathrm{C} 19 & \mathrm{H} 23 & 0.95 & \mathrm{C} 19 & \mathrm{H} 24 & 0.95 \\ \mathrm{C} 20 & \mathrm{H} 25 & 0.95 & \mathrm{C} 21 & \mathrm{H} 26 & 0.95 \\ \mathrm{C} 21 & \mathrm{H} 27 & 0.95 & \mathrm{C} 21 & \mathrm{H} 28 & 0.95 \\ \mathrm{C} 22 & \mathrm{H} 29 & 0.95 & \mathrm{C} 22 & \mathrm{H} 30 & 0.95 \\ \mathrm{C} 22 & \mathrm{H} 31 & 0.95 & \mathrm{C} 24 & \mathrm{H} 32 & 0.95 \\ \mathrm{C} 24 & \mathrm{H} 33 & 0.95 & \mathrm{C} 24 & \mathrm{H} 34 & 0.95\end{array}$

Table 4. Bond Lengths $(\AA)$

$\begin{array}{llllll}\text { atom } & \text { atom } & \text { distance } & \text { atom } & \text { atom } & \text { distance } \\ \mathrm{C} 1 & \mathrm{H} 1 & 0.95 & \mathrm{C} 1 & \mathrm{H} 2 & 0.95 \\ \mathrm{C} 3 & \mathrm{H} 3 & 0.95 & \mathrm{C} 4 & \mathrm{H} 4 & 0.95 \\ \mathrm{C} 4 & \mathrm{H} 5 & 0.95 & \mathrm{C} 5 & \mathrm{H} 6 & 0.95 \\ \mathrm{C} 5 & \mathrm{H} 7 & 0.95 & \mathrm{C} 7 & \mathrm{H} 8 & 0.95 \\ \mathrm{C} 7 & \mathrm{H} 9 & 0.95 & \mathrm{C} 8 & \mathrm{H} 10 & 0.95 \\ \mathrm{C} 8 & \mathrm{H} 11 & 0.95 & \mathrm{C} 10 & \mathrm{H} 12 & 0.95 \\ \mathrm{C} 11 & \mathrm{H} 13 & 0.95 & \mathrm{C} 13 & \mathrm{H} 14 & 0.95 \\ \mathrm{C} 14 & \mathrm{H} 15 & 0.95 & \mathrm{C} 17 & \mathrm{H} 16 & 0.95 \\ \mathrm{C} 17 & \mathrm{H} 17 & 0.95 & \mathrm{C} 17 & \mathrm{H} 18 & 0.95 \\ \mathrm{C} 18 & \mathrm{H} 19 & 0.95 & \mathrm{C} 18 & \mathrm{H} 20 & 0.95 \\ \mathrm{C} 18 & \mathrm{H} 21 & 0.95 & \mathrm{C} 19 & \mathrm{H} 22 & 0.95 \\ \mathrm{C} 19 & \mathrm{H} 23 & 0.95 & \mathrm{C} 19 & \mathrm{H} 24 & 0.95 \\ \mathrm{C} 20 & \mathrm{H} 25 & 0.95 & \mathrm{C} 21 & \mathrm{H} 26 & 0.95 \\ \mathrm{C} 21 & \mathrm{H} 27 & 0.95 & \mathrm{C} 21 & \mathrm{H} 28 & 0.95 \\ \mathrm{C} 22 & \mathrm{H} 29 & 0.95 & \mathrm{C} 22 & \mathrm{H} 30 & 0.95 \\ \mathrm{C} 22 & \mathrm{H} 31 & 0.95 & \mathrm{C} 24 & \mathrm{H} 32 & 0.95 \\ \mathrm{C} 24 & \mathrm{H} 33 & 0.95 & \mathrm{C} 24 & \mathrm{H} 34 & 0.95\end{array}$


Table 5. Bond Angles( $\left.{ }^{\circ}\right)$

$\begin{array}{llllllll}\text { atom } & \text { atom } & \text { atom } & \text { angle } & \text { atom } & \text { atom } & \text { atom } & \text { angle } \\ \text { C1 } & \text { O1 } & \text { C14 } & 109.3(2) & \mathrm{C} 3 & \text { O2 } & \text { C16 } & 116.2(3) \\ \text { C11 } & \text { O4 } & \text { C23 } & 116.8(2) & \mathrm{O} 1 & \mathrm{C} 1 & \mathrm{C} 2 & 105.1(2) \\ \mathrm{C} 1 & \mathrm{C} 2 & \mathrm{C} 3 & 125.2(3) & \mathrm{C} 1 & \mathrm{C} 2 & \mathrm{C} 15 & 110.3(3) \\ \mathrm{C} 3 & \mathrm{C} 2 & \mathrm{C} 15 & 124.4(3) & \mathrm{O} 2 & \mathrm{C} 3 & \mathrm{C} 2 & 110.5(2) \\ \mathrm{O} 2 & \mathrm{C} 3 & \mathrm{C} 4 & 107.2(3) & \mathrm{C} 2 & \mathrm{C} 3 & \mathrm{C} 4 & 111.9(3) \\ \mathrm{C} 3 & \mathrm{C} 4 & \mathrm{C} 5 & 112.9(3) & \mathrm{C} 4 & \mathrm{C} 5 & \mathrm{C} 6 & 112.9(3) \\ \mathrm{C} 5 & \mathrm{C} 6 & \mathrm{C} 7 & 109.0(3) & \mathrm{C} 5 & \mathrm{C} 6 & \mathrm{C} 15 & 106.9(3) \\ \mathrm{C} 5 & \mathrm{C} 6 & \mathrm{C} 18 & 109.8(3) & \mathrm{C} 7 & \mathrm{C} 6 & \mathrm{C} 15 & 112.1(3) \\ \mathrm{C} 7 & \mathrm{C} 6 & \mathrm{C} 18 & 110.7(3) & \mathrm{C} 15 & \mathrm{C} 6 & \mathrm{C} 18 & 108.2(3) \\ \mathrm{C} 6 & \mathrm{C} 7 & \mathrm{C} 8 & 117.0(3) & \mathrm{C} 7 & \mathrm{C} 8 & \mathrm{C} 9 & 115.6(3) \\ \mathrm{C} 8 & \mathrm{C} 9 & \mathrm{C} 10 & 112.9(2) & \mathrm{C} 8 & \mathrm{C} 9 & \mathrm{C} 13 & 113.1(2) \\ \mathrm{C} 8 & \mathrm{C} 9 & \mathrm{C} 19 & 110.3(2) & \mathrm{C} 10 & \mathrm{C} 9 & \mathrm{C} 13 & 102.0(2) \\ \mathrm{C} 10 & \mathrm{C} 9 & \mathrm{C} 19 & 110.3(3) & \mathrm{C} 13 & \mathrm{C} 9 & \mathrm{C} 19 & 107.9(3) \\ \mathrm{C} 9 & \mathrm{C} 10 & \mathrm{C} 11 & 105.2(2) & \mathrm{C} 9 & \mathrm{C} 10 & \mathrm{C} 20 & 120.9(3) \\ \mathrm{C} 11 & \mathrm{C} 10 & \mathrm{C} 20 & 113.5(3) & \mathrm{O} 4 & \mathrm{C} 11 & \mathrm{C} 10 & 114.4(2) \\ \mathrm{O} 4 & \mathrm{C} 11 & \mathrm{C} 12 & 106.3(2) & \mathrm{C} 10 & \mathrm{C} 11 & \mathrm{C} 12 & 105.5(2) \\ \mathrm{O} 6 & \mathrm{C} 12 & \mathrm{C} 11 & 124.3(3) & \mathrm{O} 6 & \mathrm{C} 12 & \mathrm{C} 13 & 126.9(3) \\ \mathrm{C} 11 & \mathrm{C} 12 & \mathrm{C} 13 & 108.6(3) & \mathrm{C} 9 & \mathrm{C} 13 & \mathrm{C} 12 & 103.2(2) \\ \mathrm{C} 9 & \mathrm{C} 13 & \mathrm{C} 14 & 113.7(2) & \mathrm{C} 12 & \mathrm{C} 13 & \mathrm{C} 14 & 111.0(2) \\ \mathrm{O} 1 & \mathrm{C} 14 & \mathrm{C} 13 & 110.7(2) & \mathrm{O} 13 & \mathrm{C} 14 & \mathrm{C} 15 & 104.6(2) \\ \mathrm{C} 13 & \mathrm{C} 14 & \mathrm{C} 15 & 112.6(2) & \mathrm{C} 2 & \mathrm{C} 15 & \mathrm{C} 6 & 125.9(3) \\ \mathrm{C} 2 & \mathrm{C} 15 & \mathrm{C} 14 & 109.6(2) & \mathrm{C} 6 & \mathrm{C} 15 & \mathrm{C} 14 & 124.5(3) \\ \mathrm{O} 2 & \mathrm{C} 16 & \mathrm{O} 3 & 123.6(3) & \mathrm{O} 2 & \mathrm{C} 16 & \mathrm{C} 17 & 111.9(3) \\ \mathrm{O} 3 & \mathrm{C} 16 & \mathrm{C} 17 & 124.5(3) & \mathrm{C} 10 & \mathrm{C} 20 & \mathrm{C} 21 & 110.7(3) \\ \mathrm{C} 10 & \mathrm{C} 20 & \mathrm{C} 22 & 114.2(3) & \mathrm{C} 21 & \mathrm{C} 20 & \mathrm{C} 22 & 108.4(3) \\ \text { O4 } & \mathrm{C} 23 & \mathrm{O} 5 & 123.5(3) & \mathrm{O} 4 & \mathrm{C} 23 & \mathrm{C} 24 & 110.9(3) \\ \mathrm{O} 5 & \mathrm{C} 23 & \mathrm{C} 24 & 125.6(3) & & & & \\ & & & & & & \end{array}$


Table 6. Bond Angles $\left({ }^{\circ}\right)$

\begin{tabular}{|c|c|c|c|c|c|c|c|}
\hline atom & atom & atom & angle & atom & atom & atom & angle \\
\hline $\mathrm{O} 1$ & $C 1$ & H1 & 110.6 & $\mathrm{O} 1$ & $\mathrm{C} 1$ & $\mathrm{II} 2$ & 110.6 \\
\hline $\mathrm{C} 2$ & $\mathrm{C} 1$ & H1 & 110.6 & $\mathrm{C} 2$ & $\mathrm{C} 1$ & $\mathrm{H} 2$ & 110.6 \\
\hline H1 & $\mathrm{C} 1$ & $\mathrm{H} 2$ & 109.5 & $\mathrm{O} 2$ & C3 & H3 & 109.1 \\
\hline $\mathrm{C} 2$ & $\mathrm{C} 3$ & H3 & 109.1 & $\mathrm{C} 4$ & C3 & H3 & 109.1 \\
\hline C3 & $\mathrm{C} 4$ & $\mathrm{H} 4$ & 108.6 & C3 & $\mathrm{C} 4$ & $\mathrm{H} 5$ & 108.6 \\
\hline $\mathrm{C} 5$ & $\mathrm{C} 4$ & $\mathrm{H} 4$ & 108.6 & C5 & $\mathrm{C} 4$ & $\mathrm{H} 5$ & 108.6 \\
\hline $\mathrm{H} 4$ & $\mathrm{C} 4$ & $\mathrm{H} 5$ & 109.4 & $\mathrm{C} 4$ & C5 & $\mathrm{H} 6$ & 108.6 \\
\hline $\mathrm{C} 4$ & $\mathrm{C} 5$ & $\mathrm{H} 7$ & 108.6 & C6 & C5 & $\mathrm{H} 6$ & 108.6 \\
\hline C6 & $\mathrm{C} 5$ & $\mathrm{H} 7$ & 108.6 & H6 & C5 & $\mathrm{H} 7$ & 109.4 \\
\hline C6 & $\mathrm{C} 7$ & $\mathrm{H} 8$ & 107.6 & C6 & $\mathrm{C} 7$ & H9 & 107.6 \\
\hline $\mathrm{C} 8$ & $\mathrm{C} 7$ & $\mathrm{H} 8$ & 107.6 & $\mathrm{C} 8$ & $\mathrm{C} 7$ & $\mathrm{H} 9$ & 107.5 \\
\hline $\mathrm{H} 8$ & $\mathrm{C} 7$ & H9 & 109.5 & $\mathrm{C} 7$ & $\mathrm{C} 8$ & $\mathrm{H} 10$ & 107.9 \\
\hline $\mathrm{C} 7$ & $\mathrm{C} 8$ & H11 & 107.9 & C9 & C8 & $\mathrm{H} 10$ & 107.9 \\
\hline C9 & $\mathrm{C} 8$ & H11 & 107.9 & H10 & C8 & H11 & 109.5 \\
\hline C9 & $\mathrm{C} 10$ & H 12 & 105.3 & C11 & C10 & H12 & 105.3 \\
\hline $\mathrm{C} 20$ & C10 & $\mathrm{H} 12$ & 105.3 & $\mathrm{O} 4$ & C11 & H13 & 110.1 \\
\hline $\mathrm{C} 10$ & C11 & H13 & 110.1 & $\mathrm{C} 12$ & C11 & H13 & 110.1 \\
\hline $\mathrm{C} 9$ & C13 & H14 & 109.6 & $\mathrm{C} 12$ & C13 & H14 & 109.6 \\
\hline C14 & $\mathrm{C} 13$ & H14 & 109.6 & $\mathrm{O} 1$ & C14 & H15 & 109.6 \\
\hline C13 & C14 & H15 & 109.6 & C15 & C14 & H15 & 109.6 \\
\hline C16 & $\mathrm{C} 17$ & H16 & 109.5 & $\mathrm{C} 16$ & $\mathrm{C} 17$ & H 17 & 109.5 \\
\hline C16 & C17 & H18 & 109.5 & H16 & C17 & H17 & 109.4 \\
\hline H16 & $\mathrm{C} 17$ & H18 & 109.5 & H17 & C17 & H 18 & 109.5 \\
\hline C6 & $\mathrm{C} 18$ & H 19 & 109.5 & C6 & C18 & H2O & 109.5 \\
\hline C6 & $\mathrm{C} 18$ & $\mathrm{H} 21$ & 109.5 & H19 & C18 & $\mathrm{H} 20$ & 109.5 \\
\hline H19 & $\mathrm{C} 18$ & H21 & 109.5 & $\mathrm{H} 20$ & C18 & H21 & 109.5 \\
\hline C9 & C19 & $\mathrm{H} 22$ & 109.5 & C9 & C19 & $\mathrm{H} 23$ & 109.5 \\
\hline C9 & C19 & $\mathrm{H} 24$ & 109.5 & $\mathrm{H} 22$ & C19 & $\mathrm{H} 23$ & 109.5 \\
\hline $\mathrm{H} 22$ & C19 & $\mathrm{H} 24$ & 109.5 & $\mathrm{H} 23$ & C19 & $\mathrm{H} 24$ & 109.5 \\
\hline C10 & $\mathrm{C} 20$ & $\mathrm{H} 25$ & 107.8 & $\mathrm{C} 21$ & $\mathrm{C} 20$ & $\mathrm{H} 25$ & 107.8 \\
\hline $\mathrm{C} 22$ & $\mathrm{C} 20$ & $\mathrm{H} 25$ & 107.8 & $\mathrm{C} 20$ & $\mathrm{C} 21$ & $\mathrm{H} 26$ & 109.5 \\
\hline $\mathrm{C} 20$ & $\mathrm{C} 21$ & $\mathrm{H} 27$ & 109.5 & $\mathrm{C} 20$ & $\mathrm{C} 21$ & $\mathrm{H} 28$ & 109.5 \\
\hline $\mathrm{H} 26$ & $\mathrm{C} 21$ & $\mathrm{H} 27$ & 109.5 & H26 & $\mathrm{C} 21$ & $\mathrm{H} 28$ & 109.5 \\
\hline $\mathrm{H} 27$ & $\mathrm{C} 21$ & H28 & 109.5 & $\mathrm{C} 20$ & $\mathrm{C} 22$ & H 29 & 109.5 \\
\hline $\mathrm{C} 20$ & $\mathrm{C} 22$ & H30 & 109.5 & $\mathrm{C} 20$ & $\mathrm{C} 22$ & Н31 & 109.5 \\
\hline $\mathrm{H} 29$ & $\mathrm{C} 22$ & $\mathrm{H} 30$ & 109.5 & $\mathrm{H} 29$ & $\mathrm{C} 22$ & H31 & 109.5 \\
\hline H30 & $(22$ & H31 & 109.4 & $\mathrm{C} 23$ & $\mathrm{C} 24$ & H32 & 109.5 \\
\hline $\mathrm{C} 23$ & $C 24$ & H33 & 109.5 & $\mathrm{C} 23$ & $\mathrm{C} 24$ & H34 & 109.5 \\
\hline H 32 & $\mathrm{C} 24$ & H33 & 109.4 & H32 & $\mathrm{C} 24$ & H34 & 109.5 \\
\hline
\end{tabular}


Table 6. Bond Angles $\left(^{\circ}\right)$ (continued)

$\begin{array}{lllllll}\text { atom } & \text { atom } & \text { atom } & \text { angle } & \text { atom } & \text { atom } & \text { atom } \\ \text { H33 } & \text { C24 } & \text { H34 } & 109.4 & & \end{array}$

Table 7. Torsion Angles $\left({ }^{\circ}\right)$

\begin{tabular}{|c|c|c|c|c|c|c|c|c|c|}
\hline atom & atom & atom & atom & angle & atom & atom & atom & atom & angle \\
\hline O1 & C1 & $\mathrm{C} 2$ & $\mathrm{C} 3$ & $-171.6(3)$ & $\mathrm{O} 1$ & C1 & $\mathrm{C} 2$ & $\mathrm{C} 15$ & $5.2(4)$ \\
\hline O1 & $\mathrm{C} 14$ & $\mathrm{C} 13$ & C9 & $-155.7(2)$ & $\mathrm{O} 1$ & C14 & $\mathrm{C} 13$ & $\mathrm{C} 12$ & $-39.9(3)$ \\
\hline 01 & C14 & $\mathrm{C} 15$ & $\mathrm{C} 2$ & $-7.5(3)$ & $\mathrm{O} 1$ & C14 & C15 & $\mathrm{C} 6$ & $173.8(3)$ \\
\hline$(0) 2$ & C 3 & $\mathrm{C} 2$ & $\mathrm{C} 1$ & $-68.7(4)$ & $\mathrm{O} 2$ & C3 & $\mathrm{C} 2$ & $\mathrm{C} 15$ & $114.9(3)$ \\
\hline$(0) 2$ & C 3 & $\mathrm{C} 4$ & $\mathrm{C} 5$ & $-87.0(3)$ & $\mathrm{O} 3$ & C16 & $\mathrm{O} 2$ & $\mathrm{C} 3$ & $-6.6(5)$ \\
\hline 0.4 & C11 & $\mathrm{C} 10$ & $\mathrm{C} 9$ & $98.2(3)$ & $\mathrm{O} 4$ & C11 & $\mathrm{C} 10$ & $\mathrm{C} 20$ & $-36.1(4)$ \\
\hline O4 & $\mathrm{C} 11$ & $\mathrm{C} 12$ & O6 & $48.0(4)$ & $\mathrm{O} 4$ & C11 & $\mathrm{C} 12$ & $\mathrm{C} 13$ & $-128.2(2)$ \\
\hline O5 & $\mathrm{C} 23$ & $\mathrm{O} 4$ & C11 & $-10.7(4)$ & $\mathrm{O} 6$ & $\mathrm{C} 12$ & $\mathrm{C} 11$ & $\mathrm{C} 10$ & $169.9(3)$ \\
\hline O6 & $\mathrm{C} 12$ & $\mathrm{C} 13$ & C9 & $-147.6(3)$ & O6 & $\mathrm{C} 12$ & $\mathrm{C} 13$ & $\mathrm{C} 14$ & $90.2(4)$ \\
\hline $\mathrm{C} 1$ & $\mathrm{O} 1$ & $\mathrm{C} 14$ & $\mathrm{C} 13$ & $-110.7(3)$ & $\mathrm{C} 1$ & $\mathrm{O} 1$ & C14 & $\mathrm{C} 15$ & $10.8(3)$ \\
\hline $\mathrm{C} 1$ & $\mathrm{C} 2$ & C 3 & $\mathrm{C} 4$ & $171.9(3)$ & $\mathrm{C} 1$ & $\mathrm{C} 2$ & $\mathrm{C} 15$ & $\mathrm{C} 6$ & $-179.9(3)$ \\
\hline C1 & $\mathrm{C} 2$ & $\mathrm{C} 15$ & $\mathrm{C} 14$ & $1.5(4)$ & $\mathrm{C} 2$ & $\mathrm{C} 1$ & $\mathrm{O} 1$ & $\mathrm{C} 14$ & $-10.0(3)$ \\
\hline $\mathrm{C} 2$ & $\mathrm{C} 3$ & $\mathrm{O} 2$ & C16 & $85.5(3)$ & $\mathrm{C} 2$ & $\mathrm{C} 3$ & $\mathrm{C} 4$ & $\mathrm{C} 5$ & $34.3(4)$ \\
\hline $\mathrm{C} 2$ & C15 & $\mathrm{C} 6$ & $\mathrm{C} 5$ & $-18.9(4)$ & $\mathrm{C} 2$ & C15 & $\mathrm{C} 6$ & $\mathrm{C} 7$ & $-138.3(3)$ \\
\hline $\mathrm{C} 2$ & C15 & $\mathrm{C} 6$ & C18 & $99.4(4)$ & $\mathrm{C} 2$ & C15 & C14 & $\mathrm{C} 13$ & $112.7(3)$ \\
\hline $\mathrm{C} 3$ & $\mathrm{O} 2$ & $\mathrm{C} 16$ & $\mathrm{C} 17$ & $175.1(2)$ & $\mathrm{C} 3$ & $\mathrm{C} 2$ & $\mathrm{C} 15$ & $\mathrm{C} 6$ & $-3.0(5)$ \\
\hline $\mathrm{C} 3$ & $\mathrm{C} 2$ & $\mathrm{C} 15$ & $\mathrm{C} 14$ & $178.3(3)$ & C3 & $\mathrm{C} 4$ & $\mathrm{C} 5$ & $\mathrm{C} 6$ & $-59.0(4)$ \\
\hline $\mathrm{C} 4$ & C3 & $\mathrm{O} 2$ & C16 & $-152.3(3)$ & $\mathrm{C} 4$ & $\mathrm{C} 3$ & $\mathrm{C} 2$ & $\mathrm{C} 15$ & $-4.4(5)$ \\
\hline $\mathrm{C} 4$ & $\mathrm{C} 5$ & $\mathrm{C} 6$ & $\mathrm{C} 7$ & $169.6(3)$ & $\mathrm{C} 4$ & $\mathrm{C} 5$ & $\mathrm{C} 6$ & $\mathrm{C} 15$ & $48.2(3)$ \\
\hline $\mathrm{C} 4$ & $\mathrm{C} 5$ & $\mathrm{C} 6$ & $\mathrm{C} 18$ & $-69.0(3)$ & $\mathrm{C} 5$ & C6 & $\mathrm{C} 7$ & $\mathrm{C} 8$ & $-178.5(2)$ \\
\hline C5 & $\mathrm{C} 6$ & $\mathrm{C} 15$ & $\mathrm{C} 14$ & $159.5(3)$ & $\mathrm{C} 6$ & $\mathrm{C} 7$ & $\mathrm{C} 8$ & $\mathrm{C} 9$ & $91.0(3)$ \\
\hline C6 & $\mathrm{C} 15$ & C14 & $\mathrm{C} 13$ & $-66.0(4)$ & $\mathrm{C} 7$ & $\mathrm{C} 6$ & $\mathrm{C} 15$ & C14 & $40.1(4)$ \\
\hline $\mathrm{C} 7$ & $\mathrm{C} 8$ & C. & $\mathrm{C} 10$ & $-156.8(3)$ & $\mathrm{C} 7$ & $\mathrm{C} 8$ & $\mathrm{C} 9$ & $\mathrm{C} 13$ & $-41.6(4)$ \\
\hline $\mathrm{C} 7$ & $\mathrm{C} 8$ & $\mathrm{CO}$ & C19 & $79.3(3)$ & $\mathrm{C} 8$ & $\mathrm{C} 7$ & $\mathrm{C} 6$ & $\mathrm{C} 15$ & $-60.4(4)$ \\
\hline $\mathrm{C} 8$ & $\mathrm{C} 7$ & C6 & $\mathrm{C} 18$ & $60.5(3)$ & $\mathrm{C} 8$ & $\mathrm{C} 9$ & $\mathrm{C} 10$ & $\mathrm{C} 11$ & $157.0(2)$ \\
\hline C8 & $\mathrm{C9}$ & $\mathrm{C} 10$ & $\mathrm{C} 20$ & $-72.9(4)$ & $\mathrm{C} 8$ & C9 & C13 & $\mathrm{C} 12$ & $-160.0(3)$ \\
\hline C8 & $\mathrm{C} 9$ & $\mathrm{C} 13$ & $\mathrm{C} 14$ & $-39.7(3)$ & $\mathrm{C} 9$ & $\mathrm{C} 10$ & $\mathrm{C} 11$ & $\mathrm{C} 12$ & $-18.3(3)$ \\
\hline $\mathrm{Cg}$ & C10 & $\mathrm{C} 20$ & $\mathrm{C} 21$ & $175.8(3)$ & $\mathrm{C} 9$ & $\mathrm{C} 10$ & $\mathrm{C} 20$ & $\mathrm{C} 22$ & $53.1(4)$ \\
\hline C9 & $\mathrm{C} 13$ & $\mathrm{C} 12$ & $\mathrm{C} 11$ & $28.4(3)$ & $\mathrm{C} 9$ & $\mathrm{C} 13$ & $\mathrm{C} 14$ & $\mathrm{C} 15$ & $87.6(3)$ \\
\hline C10 & $\mathrm{C} 9$ & $\mathrm{C} 13$ & $\mathrm{C} 12$ & $-38.5(3)$ & $\mathrm{C} 10$ & $\mathrm{C} 9$ & $\mathrm{C} 13$ & C14 & $81.8(3)$ \\
\hline C10 & $\mathrm{C} 11$ & $\mathrm{O} 4$ & $\mathrm{C} 23$ & $129.7(3)$ & $\mathrm{C} 10$ & $\mathrm{C} 11$ & $\mathrm{C} 12$ & $\mathrm{C} 13$ & $-6.3(3)$ \\
\hline C11 & $\mathrm{O} 4$ & $\mathrm{C} 23$ & $\mathrm{C} 24$ & $169.2(3)$ & $\mathrm{C} 11$ & $\mathrm{C} 10$ & $\mathrm{C} 9$ & $\mathrm{C} 13$ & $35.3(3)$ \\
\hline C11 & $\mathrm{C} 10$ & C9 & C19 & $-79.1(3)$ & $\mathrm{C} 11$ & $\mathrm{C} 10$ & $\mathrm{C} 20$ & $\mathrm{C} 21$ & $-57.8(4)$ \\
\hline C11 & $\mathrm{C} 10$ & $\mathrm{C} 20$ & $\mathrm{C} 22$ & $179.5(3)$ & $\mathrm{C} 11$ & $\mathrm{C} 12$ & $\mathrm{C} 13$ & C14 & $-93.8(3)$ \\
\hline C12 & C11 & $\mathrm{O} 4$ & $\mathrm{C} 23$ & $-114.2(3)$ & $\mathrm{C} 12$ & $\mathrm{C} 11$ & C10 & $\mathrm{C} 20$ & $-152.6(3)$ \\
\hline $\mathrm{C} 12$ & $(13$ & $\mathrm{C} 9$ & $\mathrm{C} 19$ & $77.7(3)$ & $\mathrm{C} 12$ & $\mathrm{C} 13$ & $\mathrm{C} 14$ & $\mathrm{C} 15$ & $-156.6(2)$ \\
\hline $\mathrm{C} 13$ & $\mathrm{CO}$ & $\mathrm{C} 10$ & $\mathrm{C} 20$ & $165.4(3)$ & C14 & $\mathrm{C} 13$ & C9 & C19 & $-161.9(3)$ \\
\hline C'14 & $\mathrm{C} 15$ & C6 & $\mathrm{C} 18$ & $-82.2(4)$ & $\mathrm{C} 19$ & $\mathrm{C} 9$ & $\mathrm{C} 10$ & $\mathrm{C} 20$ & $51.0(4)$ \\
\hline
\end{tabular}


Table 8. Non-bonded Contacts out to $3.75 \AA$

$\begin{array}{llllllll}\text { atom } & \text { atom } & \text { distance } & \text { ADC } & \text { atom } & \text { atom } & \text { distance } & \text { ADC } \\ \text { O1 } & \text { C18 } & 3.607(4) & 55703 & \text { O3 } & \text { C24 } & 3.393(4) & 64604 \\ \mathrm{O} 3 & \mathrm{C} 21 & 3.647(5) & 55703 & \mathrm{O} 3 & \mathrm{O} 5 & 3.725(3) & 64604 \\ \mathrm{O} 4 & \mathrm{C} 17 & 3.713(4) & 45603 & \mathrm{O} 5 & \mathrm{C} 22 & 3.268(4) & 65501 \\ \mathrm{O} 5 & \mathrm{C} 17 & 3.457(4) & 65604 & \mathrm{O} 6 & \mathrm{C} 7 & 3.554(4) & 55603 \\ \mathrm{C} 11 & \mathrm{C} 18 & 3.589(5) & 55703 & \mathrm{C} 21 & \mathrm{C} 24 & 3.713(5) & 56502\end{array}$

